JOURNAL OF THE

AMERICAN MATHEMATICAL SOCIETY

Volume 11, Number 1, January 1998, Pages 73-118

S 0894-0347(98)00248-3

\title{
THE ARASON INVARIANT AND MOD 2 ALGEBRAIC CYCLES
}

\author{
HÉLÈNE ESNAULT, BRUNO KAHN, MARC LEVINE, AND ECKART VIEHWEG
}

\section{Contents}

Introduction $\quad 73$

1. Review of the Arason invariant $\quad 75$

2. The special Clifford group $\quad 76$

3. $\mathcal{K}$-cohomology of split reductive algebraic groups $\quad 78$

4. $\mathcal{K}$-cohomology of $B G \quad 86$

5. $G L(N)$ and Cliff $(n, n) \quad 92$

6. Two invariants for Clifford bundles $\quad 95$

7. Snaking a Bloch-Ogus differential 100

8. Proof of Theorem $1 \quad 101$

9. Application to quadratic forms 102

Appendix A. Toral descent 104

Appendix B. The Rost invariant 108

Appendix C. An amusing example 113

Acknowledgements 116

References $\quad 116$

\section{INTRODUCTION}

In topology, one associates to a complex quadratic vector bundle $E$ over a topological space $X$ its Stiefel-Whitney classes

$$
w_{i}(E) \in H^{i}(X, \mathbb{Z} / 2) .
$$

These classes are essentially the only characteristic classes attached to quadratic bundles: any such bundle is classified by the homotopy class of a map $X \rightarrow B O(n, \mathbb{C})$ where $n$ is the rank of $E$. The classifying space $B O(n, \mathbb{C})$ has a tautological quadratic bundle $\mathcal{E}$ of rank $n$, and $H^{*}(B O(n, \mathbb{C}), \mathbb{Z} / 2)$ is a polynomial algebra on the Stiefel-Whitney classes of $\mathcal{E}$.

The same holds in algebraic geometry, where to any quadratic vector bundle $E$ over a $\mathbb{Z}[1 / 2]$-scheme $X$ (a vector bundle provided with a unimodular symmetric bilinear form) one can attach Stiefel-Whitney classes, living in mod 2 étale cohomology [17]

$$
w_{i}(E) \in H_{\text {ét }}^{i}(X, \mathbb{Z} / 2)
$$

Received by the editors September 13, 1996 and, in revised form, July 28, 1997.

1991 Mathematics Subject Classification. Primary 11E81; Secondary 55R40.

This research was partially supported by the DFG Forschergruppe "Arithmetik und Geometrie"; the second and third author gratefully acknowledge its hospitality. 
Here again, these classes can be defined as pull-backs of universal classes $w_{i}$ in the cohomology of the simplicial scheme $B O(n) / \mathbb{Z}[1 / 2]$. Since the latter cohomology is a polynomial algebra on the $w_{i}$ over the étale cohomology of Spec $\mathbb{Z}[1 / 2]$ [30], the $w_{i}(E)$ are essentially the only characteristic classes with values in étale cohomology with $\mathbb{Z} / 2$ coefficients attached to quadratic bundles in this context.

If we restrict to (say) virtual quadratic bundles $E$ of rank 0 such that $w_{1}(E)=$ $w_{2}(E)=0$, no new mod 2 characteristic classes arise: such bundles are classified by the infinite spinor group Spin and one can show that $H^{*}(B$ Spin, $\mathbb{Z} / 2)$ is a quotient of $H^{*}(B O, \mathbb{Z} / 2)$, both in the topological and the étale context. In particular, the $\mathrm{Wu}$ formula

$$
w_{3}=w_{1} w_{2}+S q^{1} w_{2}
$$

shows that $w_{3}(E)=0$ if $w_{1}(E)=w_{2}(E)=0$, so that there are no non-trivial degree 3 mod 2 characteristic classes for such bundles.

The situation is quite different if we restrict to quadratic bundles over schemes of the form Spec $k$, where $k$ is a field of characteristic $\neq 2$. To any $k$-quadratic form $q$, of dimension divisible by 8 and such that $w_{1}(q)=w_{2}(q)=0$, Arason [1] has attached a non-trivial invariant

$$
e^{3}(q) \in H_{\text {ét }}^{3}(k, \mathbb{Z} / 2)
$$

(see section 1 for less restrictive conditions on $q$ ). From the preceding discussion, we know that $e^{3}$ cannot be expected to extend to a 'global' invariant, i.e. one defined for quadratic bundles over arbitrary schemes. A question which arises naturally is to determine the obstruction to the existence of such a global extension. The aim of this paper is to answer this question in the case of quadratic bundles on smooth varieties over fields.

More specifically, let $X$ be a smooth, irreducible variety over $k$ (still assumed to be of characteristic $\neq 2$ ) ; let $K$ be the function field of $X$ and $E$ a quadratic bundle over $X$. The generic fiber $E_{\eta}$ corresponds to a quadratic form $q$ over $K$. Assume its Arason invariant $e=e^{3}(q) \in H_{\text {ét }}^{3}(K, \mathbb{Z} / 2)$ is defined; then one easily shows that $e$ in fact lies in the subgroup $H_{\mathrm{Zar}}^{0}\left(X, \mathcal{H}_{\text {ét }}^{3}(\mathbb{Z} / 2)\right)$. There is an exact sequence

$$
H_{\text {ét }}^{3}(X, \mathbb{Z} / 2) \longrightarrow H_{\text {Zar }}^{0}\left(X, \mathcal{H}_{\text {ét }}^{3}(\mathbb{Z} / 2)\right) \stackrel{d_{2}}{\longrightarrow} C H^{2}(X) / 2 \stackrel{\mathrm{cl}^{2}}{\longrightarrow} H_{\text {ét }}^{4}(X, \mathbb{Z} / 2)
$$

where $C H^{2}(X)$ is the second Chow group of $X$. This sequence stems from the Bloch-Ogus spectral sequence for $X$, with coefficients $\mathbb{Z} / 2[3]$, and $\mathrm{cl}^{2}$ is the cycle class map modulo 2. Our main result is the computation of $d_{2}(e) \in C H^{2}(X) / 2$.

In order to explain this result, we recall that any quadratic bundle has a Clifford invariant

$$
c(E) \in H_{\text {ét }}^{2}(X, \mathbb{Z} / 2)
$$

(a variant of $w_{2}(E)$, see Definition 2.3); in the case considered, we have

$$
c(E) \in \operatorname{Ker}\left(H_{\text {ét }}^{2}(X, \mathbb{Z} / 2) \rightarrow H_{\text {ét }}^{2}(K, \mathbb{Z} / 2)\right) \simeq \operatorname{Pic}(X) / 2 .
$$

On the other hand, the vector bundle underlying $E$ has a second $C h e r n \operatorname{class}_{2}(E) \in$ $C H^{2}(X)$. We then have:

Theorem 1. Under the above assumptions,

$$
d_{2}(e)=c_{2}(E)+c(E)^{2} \in C H^{2}(X) / 2 .
$$

Corollary 1. $\mathrm{cl}^{2}\left(c_{2}(E)+c(E)^{2}\right)=0$. 
In fact, this corollary can be obtained by more elementary means than Theorem 1: generalizing the well-known relations between Chern and Stiefel-Whitney classes which exist in topology, e.g. [49, p. 181, prob. 15-A], yields the formula $c_{2}(E)=$ $c(E)^{2}$ in $H_{\text {êt }}^{4}(X, \mathbb{Z} / 2)$.

The proof of Theorem 1 can be sketched as follows. We show that the hypothesis on $E$ implies that its class $[E] \in H_{\text {ét }}^{1}(X, O(n, n))$ lifts to a class $\widetilde{[E]} \in$ $H_{\text {ét }}^{1}(X, \operatorname{Cliff}(n, n))$, where $\operatorname{Cliff}(n, n)$ is the split special Clifford group. Now we shall associate to any $\operatorname{Cliff}(n, n)$-torsor $F$ on $X$ two characteristic classes

$$
\begin{aligned}
\gamma_{1}(F) & \in \operatorname{Pic}(X), \\
\gamma_{2}(F) & \in \mathbb{H}_{\text {êt }}^{4}(X, \Gamma(2))
\end{aligned}
$$

(see 6.7), where the right-hand-side group on the second line is Lichtenbaum's étale weight-two motivic cohomology. Recall the exact sequence ([41], [33, th. 1.1])

$$
0 \rightarrow C H^{2}(X) \rightarrow \mathbb{H}_{\text {ét }}^{4}(X, \Gamma(2)) \rightarrow H_{\text {Zar }}^{0}\left(X, \mathcal{H}_{\text {ét }}^{3}(\mathbb{Q} / \mathbb{Z}(2))\right) \rightarrow 0 .
$$

In light of this sequence, we show in Theorem 6.9 that

$$
2 \gamma_{2}(F)=c_{2}(F)+\gamma_{1}(F)^{2} \in C H^{2}(X)
$$

where $c_{2}(F)$ is the second Chern class of the $S L(2 n)$-torsor (a vector bundle) stemming from $F$. Theorem 1 follows from this identity and the identification of the map $d_{2}$ as a differential in a snake diagram.

This paper is organized as follows. In section 1 we review Arason's invariant, and in section 2 the special Clifford group. The heart of the paper is sections 3 and 4 , where we compute low-degree $\mathcal{K}$-cohomology of split reductive linear algebraic groups with simply connected derived subgroups and their classifying schemes. We collect the fruits of our labor in section 6 , where we define the invariants $\gamma_{1}(F)$ and $\gamma_{2}(F)$ and prove identity (0.1). Theorem 1 is proven in section 8 . In section 9 we give some applications to quadratic forms over a field.

There are 3 appendices. Appendix A shows how different models of the simplicial classifying scheme of a split torus yield the same $\mathcal{K}$-cohomology. Appendix B presents a construction and a characterization of the invariant defined by Serre and Rost for torsors under a simple, simply connected algebraic group $H$ over a field (see [60] and the forthcoming paper [58]): in the case of Spin, this allows this paper to be self-contained. Let us point out that our method tackles the $p$-primary part of the Rost invariant as well, in case char $k=p>0$. Finally, Appendix C compares $\mathcal{K}$-cohomology of the simplicial scheme $B H$ with that of an approximating variety $B_{r} H$ : it turns out that they don't coincide. In this last appendix, we have to stay away from the characteristic of $k$ if it is nonzero.

The group $H^{1}\left(G, \mathcal{K}_{2}\right)$ was first computed by P. Deligne at the end of the seventies [12] for any $G$, semi-simple, simply connected, and not necessarily split. Our method here is different from his.

\section{Review of the Arason invariant}

Let $k$ be a field of characteristic $\neq 2$. As is customary, we write

$$
q=\left\langle a_{1}, \ldots, a_{r}\right\rangle
$$

for the isomorphism class of the quadratic form $q(x)=a_{1} x_{1}^{2}+\cdots+a_{r} x_{r}^{2}\left(a_{i} \in k^{*}\right)$. 
Let $W(k)$ be the Witt ring of $k$ [35], [59]. The dimension of forms induces an augmentation

$$
W(k) \stackrel{\operatorname{dim}}{\longrightarrow} \mathbb{Z} / 2
$$

whose kernel, denoted by $I k$, is the ideal of even-dimensional forms. Its $n$-th power is denoted by $I^{n} k$. Since $I k$ is additively generated by the forms $\langle 1,-a\rangle\left(a \in k^{*}\right)$, $I^{n} k$ is generated by $n$-fold Pfister forms

$$
\left\langle\left\langle a_{1}, \ldots, a_{n}\right\rangle\right\rangle:=\left\langle 1,-a_{1}\right\rangle \otimes \cdots \otimes\left\langle 1,-a_{n}\right\rangle .
$$

For $n \leq 4$, there are homomorphisms

$$
e^{n}: I^{n} k / I^{n+1} k \rightarrow H^{n}(k, \mathbb{Z} / 2)
$$

characterized by $e^{n}\left(\left\langle\left\langle a_{1}, \ldots, a_{n}\right\rangle\right\rangle\right)=\left(a_{1}, \ldots, a_{n}\right):=\left(a_{1}\right) \cdots\left(a_{n}\right)$, where, for $a \in k^{*},(a) \in H^{1}(k, \mathbb{Z} / 2)$ is the class of $a$ via Kummer theory. For $n=0,1,2$, the $e^{n}$ come from elementary invariants $\operatorname{dim}, d_{ \pm}, c$ defined over the whole Witt ring $W(k)$. They can be described as follows:

- $n=0: e^{0}(q)=\operatorname{dim} q(\bmod 2)$.

- $n=1: e^{1}(q)=d_{ \pm} q:=\left((-1)^{\frac{r(r-1)}{2}} \operatorname{disc} q\right)$, where $r=\operatorname{dim} q$ and $\operatorname{disc} q=$ $a_{1} \cdots a_{r}$ if $q=\left\langle a_{1}, \ldots, a_{r}\right\rangle$.

- $n=2$ : let $C(q)$ be the Clifford algebra of $q$ and $C_{0}(q)$ the even part of $C(q)$. The algebra $C(q)$ (resp. $C_{0}(q)$ ) is a central simple algebra of exponent 2 over $k$ if $\operatorname{dim} q$ is even (resp. odd). Then

$$
e^{2}(q)=c(q)= \begin{cases}{[C(q)] \in{ }_{2} \operatorname{Br} k} & \text { if } \operatorname{dim} q \text { even, } \\ {\left[C_{0}(q)\right] \in{ }_{2} \operatorname{Br} k} & \text { if } \operatorname{dim} q \text { odd. }\end{cases}
$$

Note that ${ }_{2} \mathrm{Br} k \simeq H^{2}(k, \mathbb{Z} / 2)$ by Hilbert's Theorem 90 .

The relationship of $d_{ \pm} q$ and $c(q)$ with $w_{1}(q)$ and $w_{2}(q)$ is as follows:

- $d_{ \pm}(q)=w_{1}(q)+\frac{r(r-1)}{2}(-1)\left(\right.$ since $\left.\operatorname{disc} q=w_{1}(q)\right)$

- $c(q)=w_{2}(q)+a(-1) \cdot w_{1}(q)+b(-1,-1)$, with $a=\frac{(r-1)(r-2)}{2}$ and $b=$ $\frac{(r+1) r(r-1)(r-2)}{24}\left[35\right.$, prop. V.3.20]. In particular, if $r \equiv 0(\bmod 4), w_{1}(q)=$ $w_{2}(q)=0$ if and only if $e^{1}(q)=e^{2}(q)=0$.

The existence of $e^{3}$ was proven by Arason in his thesis [1] (see also Barge [2]): it cannot be extended to a function $W(k) \rightarrow H^{3}(k, \mathbb{Z} / 2)$ which would be natural under change of base field [1, p. 491] (see Corollary 9.3 for an unstable refinement.) Similarly, Jacob-Rost [28] and independently Szyjewski [64] proved the existence of $e^{4}$. Merkurjev [43] proved that $e^{2}$ is an isomorphism, which shows with the above remarks that $e^{3}(q)$ is defined as soon as $w_{1}(q)=w_{2}(q)=0$. Rost [56] and independently Merkurjev-Suslin [47] proved that $e^{3}$ is an isomorphism. Voevodsky has recently announced a proof that $e^{n}$ exists and is an isomorphism for all $n$ and all fields.

\section{The special Clifford group}

Recall $[17,1.9]$ that a quadratic bundle $E$ over a scheme $X$ has a Clifford algebra $C(E)$. If $E$ has even rank, $C(E)$ is an Azumaya algebra with a canonical involution $\sigma$, restricting to the identity on $E \hookrightarrow C(E)$. Recall also the Clifford group $C^{*}(E)$ $[17,1.9]$, defined as the homogeneous stabilizer of $E$ in $C(E)^{*}$ (acting by inner automorphisms). It is representable by a linear algebraic group scheme over $X$. 
When $E=\mathbb{H}\left(\mathbb{A}_{X}^{n}\right)$ is the split bundle associated with the affine $n$-space $\mathbb{A}_{X}^{n}[17$, $5.5]$, we denote this algebraic group scheme by $C^{*}(n, n)$ : it is defined over $\mathbb{Z}$.

There is a "spinor norm" homomorphism $C^{*}(E) \stackrel{\gamma_{1}}{\rightarrow} \mathbb{G}_{m}$, given by $\gamma_{1}(x)=x \sigma(x)$; as in $[17,1.9]$, we denote its kernel by $\widetilde{O}(E)$. The action of $C^{*}(E)$ on $E$ by inner automorphisms is orthogonal, hence defines a homomorphism $C^{*}(E) \rightarrow O(E)$ with kernel the center of $C^{*}(E)$, which is nothing else than $\mathbb{G}_{m}$. The situation can be summarized by the following commutative diagram with exact rows and columns:

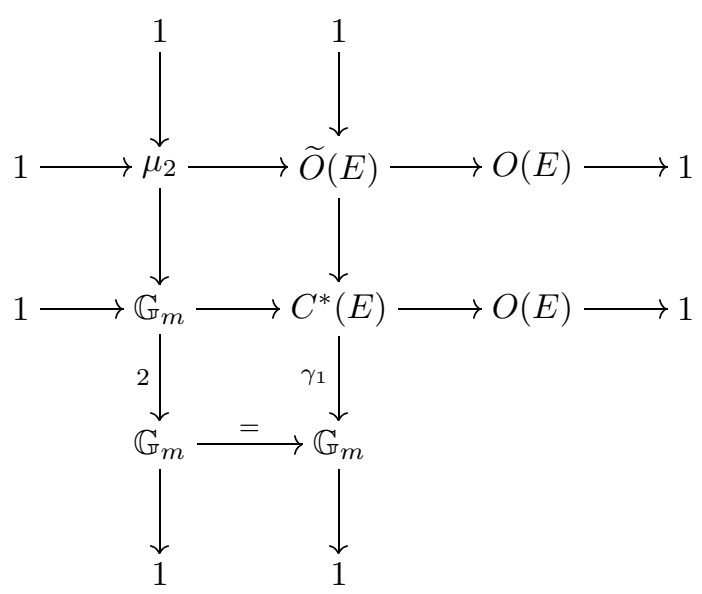

Let us denote by $\operatorname{Cliff}(E)$ the even part of $C^{*}(E)$ : this is the special Clifford group. The group $\operatorname{Cliff}(E) \cap \widetilde{O}(E)$ is nothing else than the spinor group $\operatorname{Spin}(E)$. In case $E=\mathbb{H}\left(\mathbb{A}_{X}^{n}\right)$, this is summarised by the following diagram, similar to (2.1):

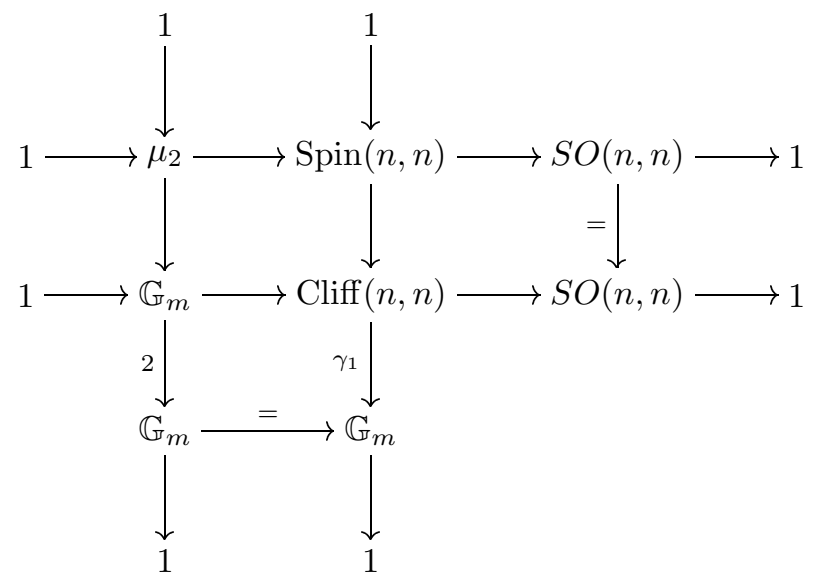

This allows one to recover Cliff in terms of Spin, if one wishes:

$$
\operatorname{Cliff}(n, n)=\operatorname{Spin}(n, n) \times \mathbb{G}_{m} / \mu_{2}
$$

via the diagonal action $-(g, t)=(-g,-t)$.

Suppose $X=\operatorname{Spec} K$. Given a quadratic form $q$, the action of $O(q)$ on the vector space underlying $q$ extends to an action of $O(q)$ on $C(q)$ by algebra automorphisms. When $q=n \mathbb{H}$ is split, $C(q) \simeq M_{2^{n}}(K)$; we denote by $\rho$ the corresponding homomorphism $O(n, n) \rightarrow P G L\left(2^{n}\right)$. Recall the invariant $c(q)$ from section 1. 
2.1. Lemma. Let $K$ be a field and let $q$ be a quadratic form with even rank $2 n$. Then $c(q)=\partial[q] \in H^{2}\left(K, \mu_{2}\right)={ }_{2} \operatorname{Br}(K)$ where $[q]$ is the class of $q$ in $H^{1}(K, O(n, n))$ and $\partial$ is the boundary map in non-abelian cohomology coming from the exact sequence

$$
1 \rightarrow \mu_{2} \rightarrow \widetilde{O}(n, n) \rightarrow O(n, n) \rightarrow 1
$$

of diagram (2.1).

Proof. This follows immediately from the commutative diagram

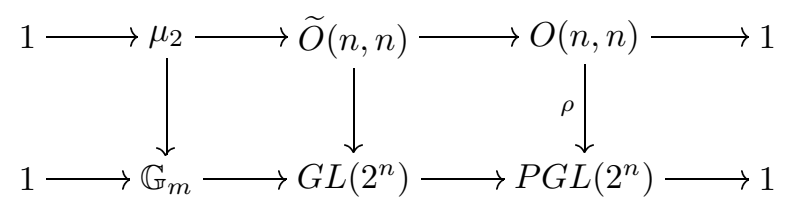

in which the middle vertical map is the natural embedding (from the definition of $C^{*}(n, n)$ and $\left.\widetilde{O}(n, n)\right)$.

2.2. Remark. Hilbert's Theorem 90 implies that the map

$$
H^{1}(K, \operatorname{Cliff}(n, n)) \rightarrow H^{1}(K, S O(n, n))
$$

is injective. In other words, over a field any quadratic form in $I^{2}$ can be refined into a Clifford bundle in a unique way (contrary to the situation for Spin-bundles). Similarly for $O(n, n)$ and $C^{*}(n, n)$.

We now extend the invariants $d_{ \pm} q$ and $c(q)$ to quadratic bundles of even rank over arbitrary schemes as follows.

2.3. Definition. If $E$ is a quadratic bundle of rank $2 n$ over $X$, then its signed discriminant $d_{ \pm} E$ is the image of $[E] \in H_{\text {ét }}^{1}(X, O(n, n))$ into $H_{\text {ét }}^{1}(X, \mathbb{Z} / 2)$ via the determinant map det $: O(n, n) \rightarrow \mu_{2} \simeq \mathbb{Z} / 2$. Its Clifford invariant $c(E)$ is the image of $[E]$ in $H_{\text {ét }}^{2}(X, \mathbb{Z} / 2)$ by the non-abelian boundary map associated with the exact sequence

$$
1 \rightarrow \mu_{2} \rightarrow \widetilde{O}(n, n) \rightarrow O(n, n) \rightarrow 1 .
$$

2.4. Remark. One can check that $d_{ \pm}(E)$ and $c(E)$ coincide with the similar invariants defined by Parimala and Srinivas in [52, 2.2 and Lemma 6]. For the latter, one proceeds as in the proof of Lemma 2.1, replacing $G L\left(2^{n}\right)$ and $P G L\left(2^{n}\right)$ by the relevant \pm -orthogonal and projective orthogonal groups, corresponding to the canonical involution carried by $C(E)$.

\section{3. $\mathcal{K}$-COHomology of SPlit Reductive Algebraic GRoups}

Let $G$ be a split reductive algebraic group over $k$. In the next section, we shall partially compute the $\mathcal{K}$-cohomology of a classifying scheme $B G$; for this we have to partially compute the $\mathcal{K}$-cohomology groups $H_{\text {Zar }}^{i}\left(G^{a}, \mathcal{K}_{j}\right)$ for various $a$. Obviously we can assume $a=1$. The method we use is the one of $[38, \S 2]$ (where it is applied to computing $K_{*}(G)$ ).

The $\mathcal{K}$-cohomology of $G$ has been computed in full by Suslin in the cases $G=$ $S L(N), G L(N)$ and $S p(2 n)[62]$. 
3.1. To start the computation of the $\mathcal{K}$-cohomology of $G$, recall that since $G$ is smooth this cohomology is given by the (co)homology of the corresponding Gersten complex. The computation in fact applies to a large extent to arbitrary "cycle modules" in the sense of Rost [57]. So we give ourselves a cycle module $K \mapsto$ $M_{*}(K)$, for $K$ running through finitely generated extensions of $k$. For any variety (smooth or not) $V / k$, we write

$$
C_{*}\left(V, M_{j}\right)
$$

for the Gersten complex

$$
\cdots \rightarrow \bigoplus_{x \in V_{(i+1)}} M_{i+j+1}(k(x)) \rightarrow \bigoplus_{x \in V_{(i)}} M_{i+j}(k(x)) \rightarrow \bigoplus_{x \in V_{(i-1)}} M_{i+j-1}(k(x)) \rightarrow \cdots
$$

where $V_{(i)}$ denotes the set of points of $V$ of dimension $i$, and $A_{i}\left(V, M_{j}\right)$ for its homology. Since $C_{*}$ is covariant for proper morphisms [57], it can be extended to simplicial $k$-schemes $V_{\bullet}$ by taking the total complex associated with the bicomplex

$$
\cdots \rightarrow C_{*}\left(V_{n+1}, M_{j}\right) \rightarrow C_{*}\left(V_{n}, M_{j}\right) \rightarrow C_{*}\left(V_{n-1}, M_{j}\right) \rightarrow \cdots
$$

provided the face maps of $V_{\bullet}$ are proper. Under this assumption we are allowed to define cycle homology of $V_{\bullet}$ as the homology of this total complex. Then there is a spectral sequence

$$
E_{p, q}^{1}=A_{q}\left(V_{p}, M_{j}\right) \Rightarrow A_{p+q}\left(V_{\bullet}, M_{j}\right)
$$

We can do the same with an augmented simplicial scheme.

3.2. The pairings $K_{i}^{M} \otimes_{\mathbb{Z}} M_{j} \rightarrow M_{i+j}$ give morphisms of complexes, for two varieties $V$ and $W[57,(14.1)]$ :

$$
C_{*}\left(V, K_{i}^{M}\right) \otimes_{\mathbb{Z}} C_{*}\left(W, M_{j}\right) \rightarrow C_{*}\left(V \times_{k} W, M_{i+j}\right)
$$

hence homomorphisms

$$
A_{m}\left(V, K_{i}^{M}\right) \otimes_{\mathbb{Z}} A_{n}\left(W, M_{j}\right) \rightarrow A_{m+n}\left(V \times_{k} W, M_{i+j}\right)
$$

and, for $i=-m$ :

$$
C H_{m}(V) \otimes_{\mathbb{Z}} A_{n}\left(W, M_{j}\right) \rightarrow A_{m+n}\left(V \times_{k} W, M_{j-m}\right)
$$

where $C H_{n}(V)$ are Chow homology groups $[19, \S 1.3]$. Putting all gradings together, we note that (3.1) refines into a morphism of complexes

$$
C_{*}\left(V, K_{*}^{M}\right) \otimes_{K_{*}^{M}(k)} C_{*}\left(W, M_{*}\right) \rightarrow C_{*}\left(V \times_{k} W, M_{*}\right)
$$

since the $C_{m}\left(V, K_{*}^{M}\right)$ and $C_{n}\left(W, M_{*}\right)$ are all modules over $K_{*}^{M}(k)$.

3.3. If $Z$ is a closed subset of $V$, one has an exact sequence of complexes

$$
0 \rightarrow C_{*}\left(Z, M_{j}\right) \rightarrow C_{*}\left(V, M_{j}\right) \rightarrow C_{*}\left(V-Z, M_{j}\right) \rightarrow 0
$$

which is canonically split as an exact sequence of graded abelian groups [57, (3.10.1)]. This yields a "localization" exact sequence $[57, \S 5]$

$$
\cdots \rightarrow A_{i}\left(Z, M_{j}\right) \rightarrow A_{i}\left(V, M_{j}\right) \rightarrow A_{i}\left(V-Z, M_{j}\right) \rightarrow A_{i-1}\left(Z, M_{j}\right) \rightarrow \cdots
$$

Putting all gradings together, we note that (3.3) gives an exact sequence of complexes

$$
0 \rightarrow C_{*}\left(Z, M_{*}\right) \rightarrow C_{*}\left(V, M_{*}\right) \rightarrow C_{*}\left(V-Z, M_{*}\right) \rightarrow 0
$$

which is split as an exact sequence of graded $K_{*}^{M}(k)$-modules. 
Suppose we have a finite closed covering $Z=\bigcup_{i} Z_{i}$ of some variety $Z$, and let $Z$. be the associated simplicial scheme. Note that all face maps come from closed immersions, hence $C_{*}\left(Z_{\bullet}, M_{*}\right)$ is defined (compare 3.1). By a well-known argument, (3.4) implies that the augmentation $Z \bullet \rightarrow Z$ gives an isomorphism on $A_{*}$, yielding a Cech spectral sequence of homological type:

$$
E_{p, q}^{1}=\bigoplus_{i_{0}<\cdots<i_{p}} A_{q}\left(Z_{i_{0}} \cap \cdots \cap Z_{i_{p}}, M_{j}\right) \Rightarrow A_{p+q}\left(Z, M_{j}\right)
$$

Suppose now that $U \subset V$ is an open subset of a variety $V$ such that $Z=V-U$ is covered by the $Z_{i}$. Considering the augmented simplicial scheme $Z \bullet \rightarrow V$, we get a spectral sequence analogous to $\left[38,(1.5)_{G}\right]$

$$
E_{p, q}^{1} \Rightarrow A_{p+q}\left(U, M_{j}\right)
$$

with

$$
E_{p, q}^{1}= \begin{cases}A_{q}\left(V, M_{j}\right) & \text { if } p=0 \\ \bigoplus_{i_{1}<\cdots<i_{p}} A_{q}\left(Z_{i_{1}} \cap \cdots \cap Z_{i_{p}}, M_{j}\right) & \text { if } p>0 .\end{cases}
$$

3.4. If $V$ is purely of dimension $d$, define $A^{i}\left(V, M_{j}\right)$ as $A_{d-i}\left(V, M_{j-d}\right)$. This cycle cohomology is contravariant for all maps to a smooth variety [57, §12]. We have homotopy invariance:

$$
A^{i}\left(V, M_{j}\right) \stackrel{\sim}{\rightarrow} A^{i}\left(W, M_{j}\right)
$$

if $V$ is equidimensional and $W \rightarrow V$ is an affine bundle [57, prop. 8.6].

3.5. Let us say that a variety $X$ over $k$ is Künneth if, for any $k$-variety $Y$ and any cycle-module $M$, the pairing of complexes (3.2) is a quasi-isomorphism. (See [29, def. 14.6] for a related definition.) The following lemma gives examples of Künneth varieties:

3.6. Lemma. (i) Speck is Künneth.

(ii) If $X$ and $Y$ are Künneth, so is $X \times_{k} Y$.

(iii) Any affine bundle over a Künneth variety is Künneth.

(iv) Let $X$ be a k-variety, $Z$ a closed subset of $X$ and $U$ the complementary open subset. If among $X, Z, U$, two are Künneth varieties, then the third is.

Proof. (i) and (ii) are trivial and (iii) follows from 3.4. To see (iv), we apply the exact sequence of complexes (3.3) to $(X, Z, U)$ and $\left(X \times_{k} Y, Z \times_{k} Y, U \times_{k} Y\right)$. Since (3.5) is split as an exact sequence of graded $K_{*}^{M}(k)$-modules, it remains exact after tensorization over $K_{*}^{M}(k)$. So we get a commutative diagram of short exact 
sequences of complexes:

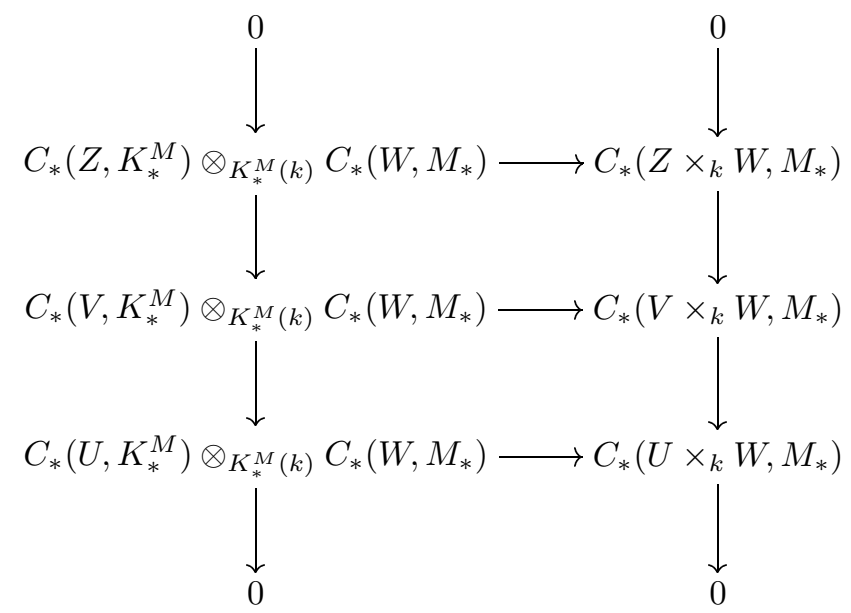

The five lemma now shows that if two rows are quasi-isomorphisms, so is the third one.

Recall that a $k$-variety $X$ is cellular if $X$ contains a closed subset $Z \neq X$ such that $X-Z \simeq \mathbb{A}_{k}^{n}$ for some $n$ and $Z$ is cellular (a recursive definition).

3.7. Proposition. a) Any cellular variety is Künneth. Moreover, if $X$ is cellular and $Y$ is arbitrary, then the $C H_{p}(X)$ are finitely generated free abelian groups and the natural map

$$
\bigoplus_{p \geq 0} C H_{p}(X) \otimes_{\mathbb{Z}} A_{n-p}\left(Y, M_{i+p}\right) \stackrel{\sim}{\rightarrow} A_{n}\left(X \times Y, M_{i}\right)
$$

is an isomorphism for all $M_{*}, n, i$.

b) A split torus is a Künneth variety.

Proof. The fact that cellular varieties and tori are Künneth follows immediately from Lemma 3.6. The fact that Chow groups of a cellular variety are finitely generated free is well-known [19, ex. 1.9.1]. It remains to show the isomorphism. For this, it suffices to show that the $A_{i}\left(X, K_{*}^{M}\right)$ are free modules over $K_{*}^{M}(k)$. This follows from

3.8. Lemma ([44], proof of prop. 1). Let $X$ be a cellular variety over $k$. Then the natural maps from 3.2

$$
C H_{i}(X) \otimes K_{j}^{M}(k) \rightarrow A_{i}\left(X, K_{j-i}^{M}\right)
$$

are isomorphisms.

3.9. If $V$ is smooth, one has

$$
A^{p}\left(V, M_{i}\right)=H_{\mathrm{Zar}}^{p}\left(V, \mathcal{M}_{i}\right)
$$

where $\mathcal{M}_{i}$ is the Zariski sheaf $U \mapsto A^{0}\left(U, M_{i}\right)$ (Gersten's conjecture, [57, cor. 6.5]). When $M_{i}$ is given by a suitable cohomology theory with supports (defined on all smooth $k$-schemes) satisfying a purity theorem, $\mathcal{M}_{i}$ can further be identified with the Zariski sheafification of $U \mapsto M_{i}(U)$. This applies to algebraic $K$-theory (Quillen [54]) and to étale cohomology with coefficients in twisted roots of unity or singular cohomology with integer coefficients when $k=\mathbb{C}$ (Bloch-Ogus [3]). 
3.10. Let $G$ be our split reductive algebraic group. We let $L_{G}=\operatorname{Hom}\left(T, \mathbb{G}_{m}\right)$ be the character group of a maximal torus $T$ of $G$. The choice of a $\mathbb{Z}$-basis of $L_{G}$ gives a $k$-isomorphism

$$
T \stackrel{\sim}{\rightarrow} \mathbb{G}_{m}^{r}
$$

with $r=\operatorname{rank} G$.

3.11. Consider the projection $G \rightarrow G / T$, with fibers $T$. Letting $X:=G / T$, one has

$$
H^{i}\left(X, \mathcal{K}_{j}\right) \simeq H^{i}\left(G / B, \mathcal{K}_{j}\right)
$$

where $B$ is a Borel subgroup, because $X \rightarrow G / B$ is an affine bundle (i.e. a torsor under a vector bundle).

The isomorphism $T \stackrel{\sim}{\rightarrow} \mathbb{G}_{m}^{r}$ defines $r$ rank one bundles $L_{1}, \ldots, L_{r}$ on $X$ such that

$$
G=L_{1}^{\times} \times_{X} \cdots \times_{X} L_{r}^{\times}
$$

where $L_{i}^{\times}$is the total space of the corresponding $\mathbb{G}_{m}$-bundle. We can then embed $G$ into the affine bundle

$$
\bar{G}:=L_{1} \times_{X} \cdots \times_{X} L_{r} .
$$

One has the following properties

$$
\bar{G}-G=\bigcup_{i=1}^{r} D_{i}
$$

where $D_{i}$ is the divisor $D_{i}=L_{1} \times_{X} \cdots \times_{X}\{0\} \times_{X} \cdots \times_{X} L_{r}$ in which the zero section $\{0\}$ is taken on the $i$-th factor of $L_{1} \times{ }_{X} \cdots \times_{X} L_{r}$.

(ii) $\left[D_{i}\right] \in \operatorname{Pic}(\bar{G})$ corresponds to $c_{1}\left(L_{i}\right) \in \operatorname{Pic}(X)$ under the isomorphism $\operatorname{Pic}(X)$ $\stackrel{\sim}{\rightarrow} \operatorname{Pic}(\bar{G})$.

3.12. We now apply the spectral sequence (3.6) to $V=\bar{G}, U=G$ and $Z_{i}=D_{i}$. This gives a spectral sequence

$$
E_{p, q}^{1}=\left\{\begin{array}{ll}
A_{q}\left(\bar{G}, M_{j}\right) & \text { if } p=0 \\
\bigoplus_{i_{1}<\cdots<i_{p}} A_{q}\left(D_{i_{1}} \cap \cdots \cap D_{i_{p}}, M_{j}\right) & \text { if } p>0
\end{array} \Rightarrow A_{p+q}\left(G, M_{j}\right) .\right.
$$

Let $d=\operatorname{dim} G=\operatorname{dim} \bar{G}$; note that $\operatorname{dim}(\bar{G}-G)=\operatorname{dim} D_{i}=d-1$ for all $i$ and similarly $\operatorname{dim}\left(D_{i_{1}} \cap \cdots \cap D_{i_{p}}\right)=d-p$ for all $p>0$. Hence the spectral sequence can be rewritten as

$E_{p, q}^{1}=\left\{\begin{array}{ll}A^{d-q}\left(\bar{G}, M_{j+d}\right) & \text { if } p=0 \\ \bigoplus_{i_{1}<\cdots<i_{p}} A^{d-p-q}\left(D_{i_{1}} \cap \cdots \cap D_{i_{p}}, M_{j+d-p}\right) & \text { if } p>0\end{array} \Rightarrow A^{d-p-q}\left(G, M_{j+d}\right)\right.$.

Since $\bar{G}$ and the $D_{i_{1}} \cap \cdots \cap D_{i_{p}}$ are all affine bundles over $X$, we can rewrite the $E^{1}$-term, using homotopy invariance 3.4

$$
E_{p, q}^{1}=\wedge^{p} L_{G} \otimes A^{d-p-q}\left(X, M_{j+d-p}\right)
$$

where $L_{G}$ is the group of characters of the split maximal torus $T$.

Since $X$ is an affine bundle over the cellular variety $G / B$ (for this, e.g. [7]), Lemma 3.8 and homotopy invariance yield the final form of the $E^{1}$-term of the above spectral sequence (after a shift on $j$ ):

$$
E_{p, q}^{1}=\wedge^{p} L_{G} \otimes C H^{d-p-q}(X) \otimes M_{j+q-d}(k) \Rightarrow A^{d-p-q}\left(G, M_{j}\right) ;
$$


compare [38, §2]. This spectral sequence is contravariant in $G$ (for group scheme homomorphisms).

It would be beyond the scope of this article to study this spectral sequence in detail, and in particular to show that it degenerates at $E^{2}$ like the similar one in [38]. We will content ourselves here with elementary remarks and low-degree computations.

3.13. From now on, we assume that $M_{j}=0$ for $j<0$. Note that this implies $A^{n}\left(X, M_{j}\right)=0$ for $n>j$, any $X$. Hence we shall care about $A^{n}\left(X, M_{j}\right)$ only for $n \leq j$.

3.14. In view of the definition of the Cech $d^{1}$-differential, the complexes

$$
\begin{aligned}
&(K .(G, d-q)) \quad \cdots \rightarrow \wedge^{p+1} L_{G} \otimes C H^{d-p-q-1}(X) \rightarrow \wedge^{p} L_{G} \otimes C H^{d-p-q}(X) \\
& \rightarrow \wedge^{p-1} L_{G} \otimes C H^{d-p-q+1}(X) \rightarrow \cdots
\end{aligned}
$$

of the $E^{1}$-terms of (3.7) can be described as follows. Let $c_{1}: L_{G} \rightarrow \operatorname{Pic}(X)$ be the homomorphism given by the first Chern class. It gives rise to a Koszul complex [27, prop. 4.3.1.2]:

$$
\begin{aligned}
\left(K_{\text {os. }}\left(c_{1}, d-q\right)\right) & \\
\cdots \rightarrow \wedge^{p+1} L_{G} \otimes \mathbf{S}^{d-p-q-1}(\operatorname{Pic}(X)) & \rightarrow \wedge^{p} L_{G} \otimes \mathbf{S}^{d-p-q}(\operatorname{Pic}(X)) \\
& \rightarrow \wedge^{p-1} L_{G} \otimes \mathbf{S}^{d-p-q+1}(\operatorname{Pic}(X)) \rightarrow \cdots .
\end{aligned}
$$

Then the natural maps $\mathbf{S}^{r}(\operatorname{Pic}(X)) \rightarrow C H^{r}(X)$ given by the intersection product provide a morphism of complexes from $K_{\text {oos. }}\left(c_{1}, d-q\right)$ to $K .(G, d-q)$.

3.15. The first two terms of $K .(G, d-q)$ and Kos. $\left(c_{1}, d-q\right)$ coincide. In particular, this yields

$$
E_{p, d-p}^{2}=\wedge^{p}\left(\operatorname{Ker} c_{1}\right)
$$

3.16. Suppose $k=\mathbb{C}$ and $M_{i}(K)=\varliminf_{\longrightarrow} H_{\text {an }}^{i}(U, \mathbb{Z})$, where $U$ runs through the open subsets of a model of $K / \mathbb{C}$. Then $\overrightarrow{M_{i}(\mathbb{C})}=0$ for $i \neq 0$ and the spectral sequence (3.7) degenerates, yielding isomorphisms

$$
H_{p}(K .(G, d-q)) \simeq H^{d-p-q}\left(G, \mathcal{H}_{\mathrm{an}}^{d-q}(\mathbb{Z})\right) .
$$

3.17. Suppose that $G$ is a torus. Then $X=\operatorname{Spec} k$, hence $C H^{i}(X)=0$ for $i>0$ and (3.7) degenerates at $E^{1}$, yielding

- $A^{p}\left(G, M_{j}\right)=0(p>0)$;

- There is a filtration on $A^{0}\left(G, M_{j}\right)$ with successive quotients $\wedge^{p} L_{G} \otimes M_{j-p}(k)$.

3.18. For $j=0,(3.7)$ gives an isomorphism $M_{0}(k) \stackrel{\sim}{\rightarrow} A^{0}\left(G, M_{0}\right)$. For $j=1$, it gives an exact sequence

$$
\begin{aligned}
0 \rightarrow M_{1}(k) \rightarrow A^{0}\left(G, M_{1}\right) \rightarrow L_{G} \otimes M_{0}(k) & \\
& \stackrel{c_{1} \otimes 1}{\longrightarrow} \operatorname{Pic}(X) \otimes M_{0}(k) \rightarrow A^{1}\left(G, M_{1}\right) \rightarrow 0 .
\end{aligned}
$$

From now on, we make the following

3.19. Assumption. $G$ is split reductive and its derived subgroup $H$ is simply connected. 
Therefore we have an exact sequence $1 \rightarrow H \rightarrow G \rightarrow S \rightarrow 1$ where

- $S$ is a split torus

- $H$ is semi-simple, simply connected and has a split torus $T_{H}$.

The unique maximal torus of $G$ containing $T_{H}$ is $T_{G}=Z(G)^{0} T_{H}$, where $Z(G)^{0}$ is the connected component of 1 in the center of $G$ [13, exposé XXII, p. 260, prop. 6.2.8]. We have an exact sequence

$$
1 \rightarrow T_{H} \rightarrow T_{G} \rightarrow S \rightarrow 1
$$

and the assumption that $H$ is simply connected implies that $L_{H} \stackrel{c_{1}}{\rightarrow} \operatorname{Pic}(X)$ is an isomorphism [14]. We also have $X_{G}=X_{H}$ (and $X_{S}=\operatorname{Spec} k$ ). This gives a split short exact sequence

$$
0 \rightarrow L_{S} \rightarrow L_{G} \stackrel{c_{1}}{\longrightarrow} \operatorname{Pic}(X) \rightarrow 0 .
$$

3.20. Proposition. Under Assumption 3.19,

(i) For $j \geq 0$, the maps $A^{0}\left(S, M_{j}\right) \rightarrow A^{0}\left(G, M_{j}\right)$ are isomorphisms. If $S=\{1\}$, we have $M_{j}(k) \stackrel{\sim}{\rightarrow} A^{0}\left(G, M_{j}\right)$ for all $j>0$.

(ii) There is for $j=1$ a short exact sequence

$$
0 \rightarrow M_{1}(k) \rightarrow A^{0}\left(G, M_{1}\right) \rightarrow L_{S} \otimes M_{0}(k) \rightarrow 0 .
$$

Moreover, $A^{1}\left(G, M_{1}\right)=0$.

(iii) For $j=2$, we have

- an exact sequence

$$
0 \rightarrow A^{1}\left(G, M_{2}\right) \rightarrow \mathbf{S}^{2}\left(L_{H}\right) \otimes M_{0}(k) \stackrel{c \otimes 1}{\longrightarrow} C H^{2}(X) \otimes M_{0}(k) \rightarrow 0
$$

where $c$ is the characteristic map $\mathbf{S}^{2}\left(L_{H}\right) \rightarrow C H^{2}(X)$;

- isomorphisms $A^{1}\left(G, M_{2}\right) \stackrel{\sim}{\rightarrow} A^{1}\left(H, M_{2}\right)$ and equalities $A^{2}\left(G, M_{2}\right)=$ $A^{2}\left(H, M_{2}\right)=0$.

Proof. To see the first claim of (i), note that 3.15 implies that the $E_{p, q}^{2}\left(S, M_{j}\right) \stackrel{\sim}{\rightarrow}$ $E_{p, q}^{2}\left(G, M_{j}\right)$ for $p+q=d$ in the spectral sequence (3.7) attached to $S$ and $G$. As observed in $3.17, E_{p, q}^{2}\left(S, M_{j}\right)=0$ for $p+q \neq d$, which implies that all differentials starting from $E_{p, q}^{2}\left(G, M_{j}\right)$ are 0 . Since no differentials arrive at $E_{p, q}^{2}\left(G, M_{j}\right)$, this means that $E_{p, q}^{2}\left(G, M_{j}\right)=E_{p, q}^{\infty}\left(G, M_{j}\right)$. The map $A^{0}\left(S, M_{j}\right) \rightarrow A^{0}\left(G, M_{j}\right)$ respects the filtrations from (3.7) and is an isomorphism on the associated graded, so it is an isomorphism. The second claim of (i) follows immediately.

(ii) follows from (3.8) and the fact that $c_{1} \otimes 1$ is surjective (3.9). We now look at the spectral sequence (3.7) for $j=2$. It follows from (3.9) and the description of $(K .(G, d-q))$ as a Koszul-like complex that

$$
\begin{array}{lll}
E_{2, d-2}^{2}=\wedge^{2}\left(L_{S}\right) \otimes M_{0}(k) & E_{1, d-2}^{2}=\operatorname{Ker} c \otimes M_{0}(k) & E_{0, d-2}^{2}=\operatorname{Coker} c \otimes M_{0}(k) \\
& E_{1, d-1}^{2}=L_{S} \otimes M_{1}(k) & \\
& & E_{0, d}^{2}=M_{2}(k) \\
E_{p, q}^{2}=0 \text { otherwise. } &
\end{array}
$$

(iii) follows easily from this computation, except for the vanishing of $A^{2}\left(G, M_{2}\right)$. To see this, suppose first that $M=K^{M}$ (Milnor $K$-theory). Then $A^{2}\left(G, M_{2}\right)=$ $C H^{2}(G)$ and this group is 0 by [42] for $G$ semi-simple classical, [38, th. 2.1] in general. Indeed, [38, th. 2.1] implies that $K_{0}(G) \simeq \mathbb{Z}$ with trivial topological filtration, and it is well-known that for any smooth variety $V$, the natural map 
$C H^{i}(V) \rightarrow \operatorname{gr}^{i} K_{0}(V)=0$ is surjective with kernel killed by $(i-1)$ !. So the characteristic map $c$ is surjective, which gives the result in general.

Let $\mathcal{C}$ be a category with finite products. Recall that a contravariant functor $T: \mathcal{C}^{o} \rightarrow$ abelian groups $\}$ is additive if $T(*)=0$, where $*$ is the final object of $\mathcal{C}$, and $T(X) \oplus T(Y) \rightarrow T(X \times Y)$ is an isomorphism for all $X, Y \in \mathcal{C}$, where the map is given by the two projections.

3.21. Corollary. Let $\mathcal{C}$ be the category of $k$-reductive groups satisfying Assumption 3.19. Then $G \mapsto A^{1}\left(G, M_{2}\right)$ is additive.

Proof. Recall that, for $G \in \mathcal{C}$, the first Chern class identifies $L_{H}$ with $\operatorname{Pic}(X)$. Let $G_{1}, G_{2} \in \mathcal{C}$, with split maximal tori $T_{1}$ and $T_{2}, G=G_{1} \times G_{2}$ with split maximal torus $T=T_{1} \times T_{2}$, and $X_{1}=G_{1} / T_{1}, X_{2}=G_{2} / T_{2}, X=G / T$. Then $X \simeq X_{1} \times X_{2}$, hence we get decompositions (using Proposition 3.7):

$$
\begin{gathered}
\mathbf{S}^{2}(\operatorname{Pic}(X)) \simeq \mathbf{S}^{2}\left(\operatorname{Pic}\left(X_{1}\right)\right) \oplus \operatorname{Pic}\left(X_{1}\right) \otimes \operatorname{Pic}\left(X_{2}\right) \oplus \mathbf{S}^{2}\left(\operatorname{Pic}\left(X_{2}\right)\right), \\
C H^{2}(X) \simeq C H^{2}\left(X_{1}\right) \oplus \operatorname{Pic}\left(X_{1}\right) \otimes \operatorname{Pic}\left(X_{2}\right) \oplus C H^{2}\left(X_{2}\right) .
\end{gathered}
$$

Moreover, the multiplication map $\mu: \mathbf{S}^{2}(\operatorname{Pic}(X)) \rightarrow C H^{2}(X)$ is diagonal with respect to these decompositions:

$$
\mu=\operatorname{diag}\left(\mu_{1}, I d, \mu_{2}\right)
$$

with obvious notation.

We shall need the following corollary in Appendices B and C:

3.22. Corollary. Let $A$ be a semi-local ring of a smooth variety over $k$. Then, for any split semi-simple simply connected algebraic group $H$ over $k$ and any cycle module $M_{*}$, there are isomorphisms:

$$
\begin{aligned}
& H_{\mathrm{Zar}}^{0}\left(H_{A}, \mathcal{M}_{i}\right) \simeq H^{0}\left(A, \mathcal{M}_{i}\right) \text { for all } i \geq 0, \\
& H_{\text {Zar }}^{1}\left(H_{A}, \mathcal{M}_{2}\right) \simeq \operatorname{Ker} c \otimes H^{0}\left(A, \mathcal{M}_{0}\right) \simeq \operatorname{Ker} c \otimes M_{0}(K), \\
& H_{\text {Zar }}^{q}\left(H_{A}, \mathcal{M}_{2}\right)=0 \text { for } q \geq 2
\end{aligned}
$$

where $c$ is the characteristic map of Proposition 3.20 (ii) and $\mathcal{M}_{i}$ is the Zariski sheaf associated to $M_{i}$ as in 3.9 .

Proof. Consider the cohomology theory with supports

$$
(X, Z) \mapsto h_{Z}^{*}(X):=H_{H \times Z}^{*}\left(H \times X, \mathcal{M}_{i}\right)
$$

(Zariski cohomology) for some $i \geq 0$. It satisfies étale excision (in the sense that $h_{Z}^{*}(X) \stackrel{\sim}{\rightarrow} h_{Z}^{*}\left(X^{\prime}\right)$ for an étale morphism $X^{\prime} \stackrel{f}{\rightarrow} X$ such that $\left.f^{-1}(Z) \stackrel{\sim}{\rightarrow} Z\right)$ and is homotopy invariant; the first fact follows from the stronger localization property (3.4) for cycle cohomology, and the second is 3.4. By the arguments of [20] (see also [10]), this cohomology theory satisfies Gersten's conjecture. In particular, for $A$ as in Corollary 3.22, with field of fractions $K$, we have exact sequences:

$$
0 \rightarrow h^{q}(A) \rightarrow h^{q}(K) \rightarrow \bigoplus_{y \in Y^{(1)}} h_{y}^{q+1}(A)
$$

where $Y=\operatorname{Spec} A$. Identifying $h_{y}^{q+1}(A)$ with $H^{q}\left(H_{k(y)}, \mathcal{M}_{i-1}\right)$ via $(3.4)$, this translates as

$$
0 \rightarrow H^{q}\left(H_{A}, \mathcal{M}_{i}\right) \rightarrow H^{q}\left(H_{K}, \mathcal{M}_{i}\right) \rightarrow \bigoplus_{y \in Y^{(1)}} H^{q}\left(H_{k(y)}, \mathcal{M}_{i-1}\right)
$$


Corollary 3.22 follows from this and the computations of Proposition 3.20 and Corollary 3.21.

3.23. Remark. Replacing $H_{\mathrm{Zar}}^{1}\left(H_{A}, \mathcal{M}_{2}\right)$ by $A^{1}\left(H_{A}, K_{2}\right)$, one gets the same answer when $A$ is an arbitrary discrete valuation ring. This can be proven by considering the localization sequence for the generic and closed points of $A$, together with the fact that $H$ can be defined over $\mathbb{Z}$ as a "groupe épinglé" (Chevalley's theorem), the latter implying that the characteristic map is independent of the base.

\section{4. $\mathcal{K}$-cohomology of $B G$}

In this section, we compute the groups $H_{\text {Zar }}^{i}\left(B G, \mathcal{M}_{j}\right)$, where $\mathcal{M}_{j}$ is as in 3.9:

- in general when $G$ is a split torus;

- for $j \leq 2$ when $G$ is as in 3.19 .

For simplicity, we sometimes drop the index zar from the groups $H_{\mathrm{Zar}}^{i}\left(B G, \mathcal{M}_{j}\right)$.

4.1. Let $X$. be a simplicial $k$-scheme such that all $X_{n}$ are smooth. Let $\mathfrak{T}$ be a Grothendieck topology over the category of schemes of finite type over $k$ (for example the Zariski or the étale topology, or the analytic topology if $k=\mathbb{C}$ ). Recall the spectral sequence [11]

$$
E_{1}^{p, q}\left(\mathcal{F}_{\bullet}\right)=\mathbb{H}_{\mathfrak{T}}^{q}\left(X_{p}, \mathcal{F}_{p}\right) \Rightarrow \mathbb{H}_{\mathfrak{T}}^{p+q}\left(X_{\bullet}, \mathcal{F}_{\bullet}\right)
$$

for any complex of simplicial sheaves $\mathcal{F}_{\bullet}$ over $X_{\bullet}$, with differential

$$
d_{1}: E_{1}^{p, q} \rightarrow E_{1}^{p+1, q}, \quad d_{1}=\sum_{i=0}^{p+1}(-1)^{i} \delta_{i}^{*} .
$$

4.2. We are especially interested in the case where $X \bullet=B G$, where $G$ is an algebraic group over $k$ and $B G=E G / G$, where $E G$ is defined by

$$
(E G)_{\ell}=G^{\Delta_{\ell}}
$$

with $\Delta_{\ell}=\{0, \ldots, \ell\}$. Here $G$ acts on $E G$ diagonally on the right:

$$
\left(g_{0}, \ldots, g_{\ell}\right) \cdot h=\left(g_{0} h, \ldots, g_{\ell} h\right)
$$

for $\left(g_{0}, \ldots, g_{\ell}\right) \in(E G)_{\ell}$ and $h \in G$. The face map $\delta_{i}$ is just "forgetting $i$ ".

4.3. Lemma. Suppose $k$ is algebraically closed, let $U$ be a unipotent subgroup of $G$, and take $u$ in $U(k)$. Then conjugation by $u$ acts by the identity on $H^{*}\left(B G, \mathcal{M}_{*}\right)$.

Proof. Let $\mu: G \times_{k} B G \rightarrow B G$ be the morphism giving the action of conjugation. As a variety $U$ is an affine space over $k$, there is a map $\varphi: \mathbb{A}_{k}^{1} \rightarrow G$ such that $u=\varphi(1), 1_{G}=\varphi(0)$. Pulling back $\mu$ by $\varphi$ gives the morphism

$$
\nu: \mathbb{A}^{1} \times_{k} B G \rightarrow B G \text {. }
$$

We have the sections

$$
i_{0}, i_{1}: B G \rightarrow \mathbb{A}^{1} \times_{k} B G
$$

with respective values 0 and 1 . The projection $p_{2}$ gives a map

$$
p_{2}^{*}: H^{*}\left(B G, \mathcal{M}_{*}\right) \rightarrow H^{*}\left(\mathbb{A}^{1} \times_{k} B G, \mathcal{M}_{*}\right) .
$$

This map is an isomorphism by homotopy invariance for the cohomology of $G^{p}$ and a comparison of spectral sequences. It follows that $\mathrm{id}=i_{0}^{*} \circ \nu^{*}=i_{1}^{*} \circ \nu^{*}=$ conjugation by $u$. 
4.4. Proposition (compare [23], lemme 1). Suppose $k$ is algebraically closed. Then the natural action of $G(k)$ on the cohomology groups $H_{\mathrm{Zar}}^{i}\left(B G, M_{j}\right)$ via inner automorphisms is trivial.

Proof. (compare loc. cit.) The group $G(k)$ is generated by the $k$-points of unipotent subgroups of $G$, together with the $k$-points of the center: if $G$ is simple the subgroup of $G$ generated by all unipotent subgroups is normal and not contained in the center, hence equal to $G$. The simple case implies the semi-simple case, and in general $G$ is generated by its derived subgroup and its center. Since $k$-points of the center obviously act trivially, the conclusion follows from Lemma 4.3.

4.5. Lemma. Let $\mathcal{C}$ be a category with finite products and $T: \mathcal{C}^{\circ} \rightarrow\{$ abelian groups $\}$ an additive functor. Let $G$ be a group object of $\mathcal{C}$. Then the cohomotopy of the cosimplicial abelian group $T(B G)$ is $T(G)$ in degree 1 and 0 elsewhere.

This is clear, since $T(B G)_{n}=T(G)^{n}$ and therefore $T(B G)$ is "dual" to $B T(G)$.

4.6. Theorem. Let $S$ be a split torus over $k$, with character group $L_{S}$. Then, for all $i, j \geq 0$, we have a canonical isomorphism

$$
\mathbf{S}^{i}\left(L_{S}\right) \otimes M_{j-i}(k) \stackrel{\sim}{\rightarrow} H_{\text {Zar }}^{i}\left(B S, \mathcal{M}_{j}\right) .
$$

Proof. By $3.9,3.17$ and (4.1), $H_{\text {Zar }}^{i}\left(B S, \mathcal{M}_{j}\right)$ is the $i$-th homotopy group of the simplicial abelian group

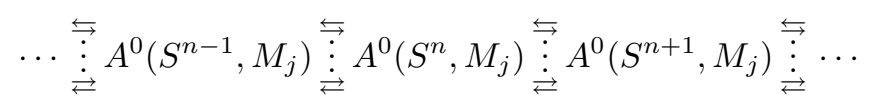

and this simplicial abelian group has a filtration whose typical quotient is

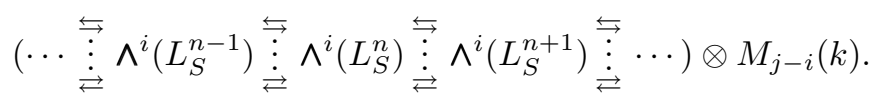

Consider the cosimplicial abelian group " $B L_{S}$ ". By Lemma 4.5 , its homotopy is $L_{S}$ in degree 1 and 0 in all other degrees. By [27, prop. 4.3.2.1], the homotopy of

$$
\cdots \underset{\vdots}{\stackrel{\leftrightarrows}{\rightleftarrows}} \wedge^{i}\left(L_{S}^{n-1}\right) \underset{:}{\stackrel{\leftrightarrows}{\rightleftarrows}} \wedge^{i}\left(L_{S}^{n}\right) \underset{\leftrightarrows}{\leftrightarrows} \wedge^{i}\left(L_{S}^{n+1}\right) \underset{\vdots}{\stackrel{\leftrightarrows}{\rightleftarrows}} \cdots
$$

is therefore $\mathbf{S}^{i}\left(L_{S}\right)$ in degree $i$ and 0 elsewhere; since this group is torsion-free, the homotopy of (4.2) is $\mathbf{S}^{i}\left(L_{S}\right) \otimes M_{j-i}(k)$ in degree $i$ and 0 elsewhere. All quotients (4.2) have their homotopy concentrated in one degree. In the spectral sequence associated to the filtration all those degrees lie on the codiagonal, hence all the differentials vanish. This yields Theorem 4.6.

4.7. Theorem. Under Assumption 3.19,

(i) For all $j$, we have isomorphisms

$$
\begin{gathered}
M_{j}(k) \stackrel{\sim}{\rightarrow} H^{0}\left(B G, \mathcal{M}_{j}\right), \\
H^{1}\left(B S, \mathcal{M}_{j}\right) \stackrel{\sim}{\rightarrow} H^{1}\left(B G, \mathcal{M}_{j}\right)
\end{gathered}
$$

and for $j \leq 2$ an exact sequence

$$
\begin{aligned}
0 \rightarrow H^{2}\left(B S, \mathcal{M}_{j}\right) \rightarrow H^{2}\left(B G, \mathcal{M}_{j}\right) & \rightarrow E_{2}^{1,1}\left(G, \mathcal{M}_{j}\right) \\
& \rightarrow H^{3}\left(B S, \mathcal{M}_{j}\right) \rightarrow H^{3}\left(B G, \mathcal{M}_{j}\right)
\end{aligned}
$$

where $E_{2}^{1,1}\left(G, \mathcal{M}_{j}\right)$ is a subgroup of $H^{1}\left(H, \mathcal{M}_{j}\right)$. 
(ii) We have

$$
\begin{aligned}
& H^{0}\left(B G, \mathcal{M}_{1}\right) \simeq M_{1}(k), \\
& H^{1}\left(B G, \mathcal{M}_{1}\right) \simeq L_{S} \otimes M_{0}(k), \\
& H^{n}\left(B G, \mathcal{M}_{1}\right)=0 \text { for } n \geq 2 .
\end{aligned}
$$

(iii) We have

$$
\begin{aligned}
& H^{0}\left(B G, \mathcal{M}_{2}\right) \simeq M_{2}(k), \\
& H^{1}\left(B G, \mathcal{M}_{2}\right) \simeq L_{S} \otimes M_{1}(k), \\
& H^{n}\left(B G, \mathcal{M}_{2}\right)=0 \text { for } n \geq 3 .
\end{aligned}
$$

(iv) The spectral sequence (4.1) yields an exact sequence

$$
0 \rightarrow E_{2}^{2,0}\left(G, \mathcal{M}_{2}\right) \rightarrow H_{\text {Zar }}^{2}\left(B G, \mathcal{M}_{2}\right) \rightarrow E_{2}^{1,1}\left(G, \mathcal{M}_{2}\right) \rightarrow 0
$$

which coincides canonically with the (exact) sequence

$$
\begin{aligned}
& 0 \rightarrow H_{\mathrm{Zar}}^{2}\left(B S, \mathcal{M}_{2}\right) \rightarrow H_{\mathrm{Zar}}^{2}\left(B G, \mathcal{M}_{2}\right) \rightarrow H_{\mathrm{Zar}}^{2}\left(B H, \mathcal{M}_{2}\right) \rightarrow 0 . \\
& \text { Moreover, } E_{2}^{1,1}\left(G, \mathcal{M}_{2}\right) \stackrel{\sim}{\rightarrow} H^{1}\left(H, \mathcal{M}_{2}\right) .
\end{aligned}
$$

Proof. Note that $E_{1}^{0, q}\left(G, \mathcal{M}_{j}\right)=0$ for $q>0$ in the spectral sequence (4.1); (i) follows from this and Proposition 3.20 (i). On the other hand, $E_{1}^{p, q}\left(G, \mathcal{M}_{j}\right)=0$ for $q>j$ (Gersten's conjecture). If $j=1$, we have moreover $E_{1}^{p, 1}=0$ for all $p$ by Proposition 3.20: this and Theorem 4.6 give (ii). Assume now $j=2$ and let us simply write $E_{r}^{p, q}(G)$ for $E_{r}^{p, q}\left(G, \mathcal{M}_{2}\right)$. This time, Proposition 3.20 (iii) implies that $E_{2}^{p, 2}(G)=0$ for all $p \geq 0$. Note also that Corollary 3.21 and Lemma 4.5 give $E_{2}^{p, 1}(G)=0$ for $p>1$ and $E_{2}^{1,1}(G)=H^{1}\left(G, \mathcal{M}_{2}\right)$. Moreover, by Proposition 3.20 (i) and Theorem 4.6, we have

$$
\mathbf{S}^{p}\left(L_{S}\right) \otimes M_{2-p}(k) \stackrel{\sim}{\rightarrow} E_{2}^{p, 0}(S) \stackrel{\sim}{\rightarrow} E_{2}^{p, 0}(G) .
$$

Finally, the only nonzero $E_{2}$-terms are $E_{2}^{1,1}(G)$ and $E_{2}^{p, 0}(G)(0 \leq p \leq 2)$; in particular, $E_{2}=E_{\infty}$. Theorem 4.7 follows easily from all these facts and Corollary 3.21 .

Let $N_{T}$ be the normalizer of $T=T_{G}$ in $G$, which we let act on $G$ by conjugation. The Weyl group $W(G)$ is by definition the quotient $N_{T} / T$. The actions of $N_{T}$ on $G$ and $T$ extend to actions on $E G$ and $E T$, giving an action of $N_{T}$ on $B G$ and $B T$.

Let $k_{s}$ be a separable closure of $k$. The restriction map

$$
H_{\text {Zar }}^{i}\left(B G / k_{s}, K_{i}^{M}\right) \rightarrow H_{\text {Zar }}^{i}\left(B T / k_{s}, K_{i}^{M}\right)(i \leq 2)
$$

is $N_{G}(T)\left(k_{s}\right)$-equivariant; by Proposition 4.4 , the action of the latter group on $H_{\mathrm{Zar}}^{i}\left(B G / \bar{k}, K_{i}^{M}\right)$ is trivial. On the other hand, since $T$ is commutative, the action of $T$ on $E T$ by conjugation is trivial, hence the $N_{T}\left(k_{s}\right)$-action on $H_{\mathrm{Zar}}^{i}\left(B T / \bar{k}, K_{i}^{M}\right)$ descends to an action of $W(G)$. It follows that the image of (4.3) is contained in the Weyl invariants $H_{\text {Zar }}^{i}\left(B T / \bar{k}, K_{i}^{M}\right)^{W(G)}$. By Theorem $4.7, H_{\text {Zar }}^{i}\left(B G / k, K_{i}^{M}\right) \rightarrow$ $H_{\mathrm{Zar}}^{i}\left(B G / \bar{k}, K_{i}^{M}\right)$ and $H_{\mathrm{Zar}}^{i}\left(B T / k, K_{i}^{M}\right) \rightarrow H_{\mathrm{Zar}}^{i}\left(B T / \bar{k}, K_{i}^{M}\right)$ are isomorphisms, hence the image of $H_{\mathrm{Zar}}^{i}\left(B G / k, K_{i}^{M}\right) \rightarrow H_{\mathrm{Zar}}^{i}\left(B T / k, K_{i}^{M}\right)$ is also contained in the 
Weyl invariants. We are now all set to prove:

4.8. Theorem. Under Assumption 3.19, restriction to the maximal torus $T$ of $G$ yields a chain of isomorphisms

$$
\begin{aligned}
H_{\mathrm{Zar}}^{i}\left(B G, \mathcal{M}_{j}\right) & \stackrel{\sim}{\leftarrow} H_{\mathrm{Zar}}^{i}\left(B G, \mathcal{K}_{i}^{M}\right) \otimes M_{j-i}(k) \\
& \stackrel{\sim}{\rightarrow} H_{\mathrm{Zar}}^{i}\left(B T, \mathcal{K}_{i}^{M}\right)^{W(G)} \otimes M_{j-i}(k) \stackrel{\sim}{\leftarrow} \mathbf{S}^{i}\left(L_{G}\right)^{W(G)} \otimes M_{j-i}(k)
\end{aligned}
$$

for $0 \leq i \leq j \leq 2$, where $W(G)$ is the Weyl group of $G$. These isomorphisms are natural in $G$.

Proof. The left isomorphism follows from Theorem 4.7 and the right one from Theorem 4.6. It remains to prove that the middle map is an isomorphism. It suffices to do this for $M_{*}=K_{*}^{M}$ and $j=i$. We proceed in two steps:

Step 1. $G$ is semi-simple. The cases $i=0,1$ are trivial. We compute the $\mathcal{K}$ cohomology of $B T$ via the spectral sequence associated to its simplicial model $E G / T$ (see Example A.6). The $E_{1}$-term of this spectral sequence is

$$
E_{1}^{p, q}=H_{\text {Zar }}^{q}\left(G / T \times G^{p}, \mathcal{K}_{2}\right) .
$$

We have $E_{1}^{p, q}=0$ for $q>2$. Since $X=G / T$ is an affine bundle over a cellular variety, Propositions 3.7 a) and 3.20 give isomorphisms:

$$
\begin{aligned}
& E_{1}^{p, 0}=K_{2}(k), \\
& E_{1}^{p, 1}=\operatorname{Pic}(X) \otimes K_{1}(k) \oplus H_{\mathrm{Zar}}^{1}\left(G^{p}, \mathcal{K}_{2}\right), \\
& E_{1}^{p, 2}=C H^{2}(X) .
\end{aligned}
$$

It follows that $E_{2}^{p, q}=0$, except for

$$
\begin{aligned}
& E_{2}^{0,2}=C H^{2}(X), \\
& E_{2}^{0,1}=\operatorname{Pic}(X) \otimes K_{1}(k), \quad E_{2}^{1,1}=H_{\text {Zar }}^{1}\left(G, \mathcal{K}_{2}\right), \\
& E_{2}^{0,0}=K_{2}(k) .
\end{aligned}
$$

We therefore get a short exact sequence

$$
0 \rightarrow H_{\text {Zar }}^{1}\left(G, \mathcal{K}_{2}\right) \rightarrow H_{\text {Zar }}^{2}\left(B T, \mathcal{K}_{2}\right) \rightarrow C H^{2}(X) \rightarrow 0
$$

and comparing with the spectral sequence for $B G$, it is clear that the isomorphism of Theorem 4.7 identifies the first map with the restriction map

$$
H_{\text {Zar }}^{2}\left(B G, \mathcal{K}_{2}\right) \rightarrow H_{\text {Zar }}^{2}\left(B T, \mathcal{K}_{2}\right) .
$$

The exact sequence (4.4) shows that $\operatorname{Coker}\left(H_{\text {Zar }}^{2}\left(B G, \mathcal{K}_{2}\right) \rightarrow H_{\text {Zar }}^{2}\left(B T, \mathcal{K}_{2}\right)\right)$ is torsion-free and by Theorem $4.6 \operatorname{Im}\left(H_{\text {Zar }}^{2}\left(B G, \mathcal{K}_{2}\right) \rightarrow H_{\text {Zar }}^{2}\left(B T, \mathcal{K}_{2}\right)\right)$ is contained in the Weyl invariants $H_{\text {Zar }}^{2}\left(B T, \mathcal{K}_{2}\right)^{W} \simeq \mathbf{S}^{2}\left(L_{G}\right)^{W}$.

On the other hand, by Proposition 3.20, $H_{\mathrm{Zar}}^{1}\left(G, \mathcal{K}_{2}\right)$ is the kernel of the characteristic map $c: \mathbf{S}^{2}\left(L_{G}\right) \rightarrow C H^{2}(X)$ and Demazure identified this kernel with $\mathbf{S}^{2}\left(L_{G}\right)^{W}$ ([14, cor. 2 to prop. 3] and [15], completed by [61]).

It follows that the map $H_{\text {Zar }}^{2}\left(B G, \mathcal{K}_{2}\right) \rightarrow H_{\text {Zar }}^{2}\left(B T, \mathcal{K}_{2}\right)^{W}$ is an injection with torsion-free cokernel between two abelian groups of the same rank. Therefore it must be surjective.

Step 2. The general case. We need a lemma:

4.9. Lemma. Let $W$ be a finite group acting on a finitely generated free $\mathbb{Z}$-module $A$. Let $B \subseteq A$ be a subgroup such that $W$ acts trivially on $B$ and $C:=A / B$ is free. 
Then

(i) The sequence

$$
0 \rightarrow B \rightarrow A^{W} \rightarrow C^{W} \rightarrow 0
$$

is exact.

(ii) If $C^{W}=0$, the sequence

$$
0 \rightarrow \mathbf{S}^{2}(B) \rightarrow \mathbf{S}^{2}(A)^{W} \rightarrow \mathbf{S}^{2}(C)^{W}
$$

is exact.

Proof. The first claim follows from the cohomology exact sequence and the equality $H^{1}(W, B)=\operatorname{Hom}(W, B)=0$. To see the second one, consider the complex of $W$ modules

$$
0 \rightarrow \mathbf{S}^{2}(B) \rightarrow \mathbf{S}^{2}(A) \rightarrow \mathbf{S}^{2}(C) \rightarrow 0
$$

This complex is acyclic, except at $\mathbf{S}^{2}(A)$ where its cohomology is $B \otimes C$. We have two hypercohomology spectral sequences

$$
I_{1}^{p, q}=H^{q}\left(W, K^{p}\right) \Rightarrow \mathbb{H}^{p+q}(W, K) \Leftarrow I I_{2}^{p, q}=H^{p}\left(W, H^{q}(K)\right) .
$$

The spectral sequence $I I$ degenerates, yielding a spectral sequence

$$
I_{1}^{p, q}=H^{q}\left(W, K^{p}\right) \Rightarrow H^{p+q-1}(W, B \otimes C) .
$$

Since $C^{W}=0$ and $B$ is free, $(B \otimes C)^{W}=0$ too. So we get $I_{2}^{0,0}=I_{2}^{1,0}=0$ and the claim follows.

End of proof of Theorem 4.8. We check it case by case with the help of Theorems 4.6 and 4.7. The case $i=0$ is trivial. Note that $W(G)=W(H)[26$, p. 181, Lemma 29.5] and $\left(L_{H}\right)^{W(H)}=0$ since $H$ is semi-simple. This yields immediately the case $i=1$. As for $i=2$, it follows from the commutative diagram

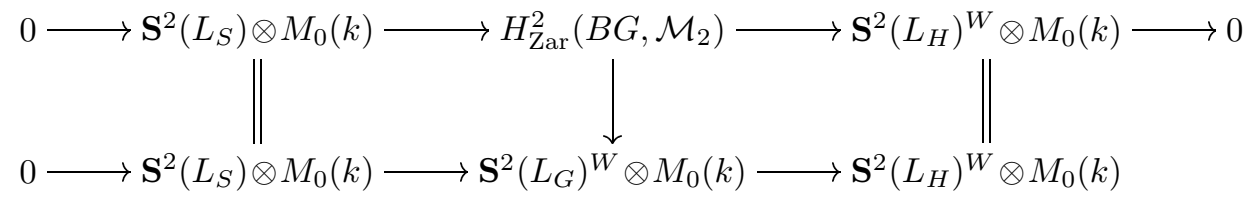

where the top row is exact by Theorem 4.7 and the bottom row is exact by Lemma 4.9 (note that $\mathbf{S}^{2}\left(L_{S}\right), \mathbf{S}^{2}\left(L_{G}\right)^{W}$ and $\mathbf{S}^{2}\left(L_{H}\right)^{W}$ are torsion-free).

4.10. Remark. The proof of Theorem 4.8 implies that for a semi-simple group $G$ the exact sequence (4.4) is up to isomorphism the same as the one in Proposition 3.20 (iii).

For a simple group $G$, it is well-known that the Weyl invariants $\mathbf{S}^{2}\left(L_{G}\right)^{W}$ have rank 1 . In fact, the representation of $W$ on $L_{G}$ is irreducible over $\mathbb{C}$ and hence

$\operatorname{dim}\left(\mathbf{S}^{2}\left(L_{G}\right)^{W}\right)+\operatorname{dim}\left(\wedge^{2}\left(L_{G}\right)^{W}\right)=\operatorname{dim}\left(\left(L_{G} \otimes L_{G}\right)^{W}\right)=\operatorname{dim}\left(\left(L_{G} \otimes\left(L_{G}\right)^{\vee}\right)^{W}\right)=1$.

On the other hand, $W$ cannot act as a symplectic representation as $L_{G}$ is defined over $\mathbb{Q}$, hence $\operatorname{dim}\left(\wedge^{2}\left(L_{G}\right)^{W}\right)=0$.

We now compare $K$-cohomology with analytic cohomology. This will be used in Appendices B and C. 
4.11. Theorem. Under Assumption 3.19, there are isomorphisms

$$
\begin{aligned}
& H^{1}\left(B G, \mathcal{K}_{1}\right) \stackrel{\sim}{\rightarrow} H_{\text {an }}^{2}(B G(\mathbb{C}), \mathbb{Z}), \\
& H^{2}\left(B G, \mathcal{K}_{2}\right) \stackrel{\sim}{\rightarrow} H_{\text {an }}^{4}(B G(\mathbb{C}), \mathbb{Z})
\end{aligned}
$$

which are natural with respect to algebraic group homomorphisms.

The proof will be in four steps.

Step 1. For $i=1,2, H^{i}\left(B G, \mathcal{K}_{i}\right)$ is invariant under base change.

This is clear from Theorem 4.8. More precisely, choose a Chevalley model of $G$ over $\mathbb{Z}$ [13], that we still denote by $G$, and choose a split maximal torus $T$ of this model as well. Then, for $i=1,2$, the functor $A \mapsto\left(\mathbf{S}^{i}\left(X\left(T_{A}\right)\right)\right)^{W}$ from commutative rings to abelian groups defines a constant sheaf for the Zariski topology, where $X\left(T_{A}\right)$ is the group of characters of $T_{A}$. By Theorem $4.8,\left(\mathbf{S}^{2}\left(X\left(T_{k}\right)\right)\right)^{W}$ is naturally isomorphic to $H^{2}\left(B G / k, \mathcal{K}_{2}\right)$ when $k$ is a field. This argument allows us in particular to pass from characteristic 0 to characteristic $p$ via some discrete valuation ring of unequal characteristic.

This shows that we may assume $k=\mathbb{C}$ in Theorem 4.11.

Step 2. For $i=1,2$, there is a natural map of Zariski sheaves $\mathcal{K}_{i} \rightarrow \mathcal{H}_{\mathrm{an}}^{i}(\mathbb{Z})$, and this map induces isomorphisms $H^{i}\left(B G, \mathcal{K}_{i}\right) \stackrel{\sim}{\rightarrow} H^{i}\left(B G, \mathcal{H}_{\mathrm{an}}^{i}(\mathbb{Z})\right)$.

For $i=1$, the map is given by the composite map of presheaves

$$
\Gamma\left(U, \mathcal{O}_{U}^{*}\right) \rightarrow H_{\mathrm{an}}^{0}\left(U, \mathbb{G}_{m}\right) \rightarrow H_{\mathrm{an}}^{1}(U, \mathbb{Z})
$$

where the second map comes from the exponential exact sequence

$$
0 \rightarrow \mathbb{Z} \stackrel{2 \pi i}{\longrightarrow} \mathbb{G}_{a} \stackrel{\exp }{\longrightarrow} \mathbb{G}_{m} \rightarrow 1 .
$$

This induces a composite

$$
\mathcal{K}_{1} \otimes \mathcal{K}_{1} \rightarrow \mathcal{H}_{\text {an }}^{1}(\mathbb{Z}) \otimes \mathcal{H}_{\text {an }}^{1}(\mathbb{Z}) \rightarrow \mathcal{H}_{\text {an }}^{2}(\mathbb{Z})
$$

in which the last map is cup-product. Since $H_{\text {an }}^{2}\left(\mathbb{A}_{\mathbb{C}}^{1}-\{0,1\}, \mathbb{Z}\right)=0$, this composite factors through $\mathcal{K}_{2}$. Now that we have comparison maps, the claim follows once again from Theorem 4.7.

Step 3. For $i=1,2$, there is a natural map $H^{i}\left(B G, \mathcal{H}_{\mathrm{an}}^{i}(\mathbb{Z})\right) \rightarrow H_{\mathrm{an}}^{2 i}(B G, \mathbb{Z})$.

Indeed, for $p+q=2$ or 4 , we have $H^{p}\left(B G, \mathcal{H}_{\mathrm{an}}^{q}(\mathbb{Z})\right)=0$ for $p>q$ by Theorem 4.7. The Bloch-Ogus spectral sequence then yields the desired homomorphism.

Step 4. The map of Step 3 is an isomorphism.

Indeed, by Theorem 4.7 (iv), we have $H^{p}\left(B G, \mathcal{H}_{\mathrm{an}}^{4-p}(\mathbb{Z})\right)=0$ for $p=0,1$.

4.12. Remark. Theorem 4.7 shows that, for any cycle module $M$,

$$
H^{i}\left(B G, \mathcal{K}_{i}\right) \otimes M_{j-i}(k) \rightarrow H^{i}\left(B G, \mathcal{M}_{j}\right)
$$

is an isomorphism for $0 \leq j \leq 2$. Together with Theorem 4.11, this yields canonical isomorphisms

$$
H^{i}\left(B G, \mathcal{M}_{j}\right) \simeq H_{\mathrm{an}}^{2 i}(B G(\mathbb{C}), \mathbb{Z}) \otimes M_{j-i}(k)
$$

for $0 \leq j \leq 2$. 


\section{5. $G L(N)$ AND $\operatorname{Cliff}(n, n)$}

In this section, we use Theorem 4.8 to compute explicitly the lower $\mathcal{K}$-cohomology of $B G$, where $G=G L(N), S L(N)$, $\operatorname{Cliff}(n, n)$ and $\operatorname{Spin}(n, n)$.

5.1. $\mathbf{S L}(\mathbf{N})$ and $\mathbf{G L}(\mathbf{N})$. We take as maximal split torus for $G L(N)$ the group $T$ of diagonal matrices and for $S L(N)$ diagonal matrices $T_{0}$ with determinant 1 . If $x_{1}, \ldots, x_{N}$ is the corresponding basis of characters of $L_{G L(N)}$, then $L_{S L(N)}=$ $L_{G L(N)} /\left\langle\sum x_{i}\right\rangle$. The Weyl group $W=\mathfrak{S}_{N}$ acts by permutation of the $x_{i}$; it follows that $\mathbf{S}\left(L_{G L(N)}\right)^{W}$ is the free polynomial algebra on the elementary symmetric functions $c_{r}$ of the $x_{i}$ and that $\mathbf{S}\left(L_{S L(N)}\right)^{W}$ is its quotient by the ideal generated by $c_{1}=\sum x_{i}$. In particular:

$$
\begin{aligned}
L_{G L(N)}^{W} & =\mathbb{Z} c_{1}, & L_{S L(N)}^{W}=0, \\
\mathbf{S}^{2}\left(L_{G L(N)}\right)^{W} & =\mathbb{Z} c_{1}^{2} \oplus \mathbb{Z} c_{2}, & \mathbf{S}^{2}\left(L_{S L(N)}\right)^{W}=\mathbb{Z} c_{2} .
\end{aligned}
$$

The restriction map induces homomorphisms $H^{i}\left(B G L(N), \mathcal{K}_{j}\right) \rightarrow H^{i}\left(B T, \mathcal{K}_{j}\right)^{W}$ and $H^{i}\left(B S L(N), \mathcal{K}_{j}\right) \rightarrow H^{i}\left(B T_{0}, \mathcal{K}_{j}\right)^{W}$, which are seen to be isomorphisms by Theorem 4.8. By Theorem 4.6 and the above computation, we get:

5.2. Theorem. For $N \geq 1$, restriction to the maximal torus yields isomorphisms:

$$
\begin{array}{ll}
H^{1}\left(B G L(N), \mathcal{K}_{1}\right)=\mathbb{Z} c_{1}, & H^{1}\left(B S L(N), \mathcal{K}_{1}\right)=0 \\
H^{2}\left(B G L(N), \mathcal{K}_{2}\right)=\mathbb{Z} c_{1}^{2} \oplus \mathbb{Z} c_{2}, & H^{2}\left(B S L(N), \mathcal{K}_{2}\right)=\mathbb{Z} c_{2} .
\end{array}
$$

5.3. Proposition. The Whitney formula holds for $c_{1}$ and $c_{2}$ : for $M, N \geq 1$ one has

$$
\begin{aligned}
& \rho^{*} c_{1}=c_{1} \times 1+1 \times c_{1}, \\
& \rho^{*} c_{2}=c_{2} \times 1+c_{1} \times c_{1}+1 \times c_{2}
\end{aligned}
$$

where $\rho$ is the embedding $G L(M) \times G L(N) \hookrightarrow G L(M+N)$. In particular, $c_{1}$ and $c_{2}$ are stable.

Proof. This can be proven by restriction to the maximal torus (Theorem 4.8) or by reduction to topology (Theorem 4.11).

5.4. Remark. This shows that the classes $c_{1}, c_{2}$ of Theorem 5.2 coincide with the Chern classes defined by Gillet in [21]. For $c_{1}$, reduce by stability to the tautological case of $G L(1)$. For $c_{2}$, reduce by Theorem 4.8 and the Whitney formula for the Gillet classes to the case of $c_{1}$.

\section{5. $\operatorname{Spin}(\mathbf{n}, \mathbf{n})$ and $\operatorname{Cliff}(\mathbf{n}, \mathbf{n})$. We have the following}

5.6. Proposition. Let $q=n \mathbb{H}$, where $\mathbb{H}$ is the quadratic form $x y$. Let $\left(e_{1}, f_{1}, \ldots\right.$, $\left.e_{n}, f_{n}\right)$ be the corresponding basis of the space underlying $q$. Then, in $\operatorname{Cliff}(n \mathbb{H})=$ $\operatorname{Cliff}(n, n)$, the assignment

$$
\left(t_{0}, t_{1}, \ldots, t_{n}\right) \mapsto t_{0}\left(t_{1} e_{1}+f_{1}\right)\left(e_{1}+f_{1}\right) \ldots\left(t_{n} e_{n}+f_{n}\right)\left(e_{n}+f_{n}\right)
$$

defines an isomorphism $\mathbb{G}_{m}^{n+1} \underset{\sim}{\stackrel{\tau}{\sim}} T$ of $\mathbb{G}_{m}^{n+1}$ onto a split maximal torus $T$. We have:

(i) $\gamma_{1} \circ \tau\left(t_{0}, \ldots, t_{n}\right)=t_{0}^{2} t_{1} \ldots t_{n}$, where $\gamma_{1}$ is the spinor norm of section 2 (see diagram (2.2)).

(ii) $\psi \circ \tau\left(t_{0}, \ldots, t_{n}\right)=\operatorname{diag}\left(t_{1}, t_{1}^{-1}, \ldots, t_{n}, t_{n}^{-1}\right)$, where $\psi: \operatorname{Cliff}(n, n) \rightarrow S L(2 n)$ is the natural map. 
(iii) For $n \geq 2$, the Weyl group $W(\operatorname{Cliff}(n, n))$ is isomorphic to the subgroup of the wreath product $\mathfrak{S}_{n} \prec \mu_{2}=\mathfrak{S}_{n} \ltimes\{ \pm 1\}^{n}$ consisting of elements $\left(\sigma, \varepsilon_{1}, \ldots, \varepsilon_{n}\right)$ such that $\varepsilon_{1} \ldots \varepsilon_{n}=1$. For $n=1$, it is trivial.

(iv) Suppose $n \geq 2$. Via the isomorphism $\tau, W(\operatorname{Cliff}(n, n))$ acts on $\mathbb{G}_{m}^{n+1}$ as follows:

$$
\begin{aligned}
& \sigma\left(t_{0}, t_{1}, \ldots, t_{n}\right)=\left(t_{0}, t_{\sigma(1)}, \ldots, t_{\sigma(n)}\right), \\
& \varepsilon\left(t_{0}, t_{1}, \ldots, t_{n}\right)=\left(t_{0} \prod_{\varepsilon_{i}=-1} t_{i}, t_{1}^{\varepsilon_{1}}, \ldots, t_{n}^{\varepsilon_{n}}\right)
\end{aligned}
$$

where $\sigma \in \mathfrak{S}_{n}$ and $\varepsilon=\left(\varepsilon_{1}, \ldots, \varepsilon_{n}\right)$ (with $\left.\prod \varepsilon_{i}=1\right)$.

Proof. Let us record the identities in the Clifford algebra of $n \mathbb{H}$ :

$$
\begin{gathered}
e_{i}^{2}=f_{i}^{2}=0, \\
e_{i} e_{j}=-e_{j} e_{i} ; f_{i} f_{j}=-f_{j} f_{i}, \\
e_{i} f_{j}=-f_{j} e_{i}(i \neq j), \\
e_{i} f_{i}+f_{i} e_{i}=1, \\
e_{i} f_{i} e_{i}=e_{i} ; f_{i} e_{i} f_{i}=f_{i} .
\end{gathered}
$$

We first show that $\tau$ is a homomorphism. Since the $e_{i}$ and $f_{i}$ with different indices anticommute, the $\left(t_{i} e_{i}+f_{i}\right)\left(e_{i}+f_{i}\right)$ mutually commute and we may assume $n=1$ and obviously $t_{0}=1$. Let us drop the indices ${ }_{1}$ for simplicity. We have

$$
\begin{gathered}
(t e+f)(e+f)=t e f+f e, \\
(s e f+f e)(t e f+f e)=s t(e f)^{2}+(f e)^{2}=s t e f+f e .
\end{gathered}
$$

Similarly, to compute $\gamma_{1} \circ \tau$ and $\psi \circ \tau$, we may assume $n=1$ and $t_{0}=1$. We have:

$$
\gamma_{1}((t e+f)(e+f))=(t e+f)(e+f)(e+f)(t e+f)=(t e+f)^{2}=t .
$$

On the other hand,

$$
\begin{gathered}
(t e f+f e) e(t e f+f e)^{-1}=(t e f+f e) e\left(t^{-1} e f+f e\right)=t e f e f e=t e \\
(t e f+f e) f(t e f+f e)^{-1}=(t e f+f e) f\left(t^{-1} e f+f e\right)=t^{-1} f e f e f=t^{-1} f
\end{gathered}
$$

and, for $v$ orthogonal to $\langle e, f\rangle$ :

$$
\begin{aligned}
(t e f+f e) v(t e f+f e)^{-1} & =(t e f+f e) v\left(t^{-1} e f+f e\right) \\
& =v(t e f+f e)\left(t^{-1} e f+f e\right)=v
\end{aligned}
$$

The composition $\mathbb{G}_{m}^{n+1} \stackrel{\tau}{\longrightarrow} T \stackrel{\left(\psi, \gamma_{1}\right)}{\longrightarrow} T_{S L(2 n)} \times \mathbb{G}_{m}$ obviously has kernel $\mu_{2} \times$ $(1, \ldots, 1)$, and scalar multiplication acts faithfully on $C(n \mathbb{H})$, so that $\tau$ is injective. Since the dimension of a maximal torus of $\operatorname{Spin}(n, n)$ or $S O(n, n)$ is $n, \tau$ is also surjective.

For $n \geq 2$, the Weyl group of $\operatorname{Cliff}(n, n)$ is the same as that of its derived subgroup $\operatorname{Spin}(n, n)$. This Weyl group is classically known [6, ch. VI, planche IV, $(\mathrm{X})]$. For $n=1, \operatorname{Cliff}(n, n) \simeq \mathbb{G}_{m} \times \mathbb{G}_{m}$, so $W=1$. Finally, let us prove the last claim. It suffices to observe that $\sigma$ is represented by an element of $\operatorname{Cliff}(n, n)$ that maps $\left(e_{i}, f_{i}\right)$ to $\pm\left(e_{\sigma(i)}, f_{\sigma(i)}\right)$ by conjugation (for $\sigma=(1,2)$, we may choose for such an element $\left.\left(e_{1}+e_{2}\right)\left(f_{1}+f_{2}\right)-1\right)$ and that $\varepsilon$ is represented by an element of 
Cliff $(n, n)$ that exchanges $e_{i}$ and $f_{i}$ exactly for those $i$ such that $\varepsilon_{i}=-1$ (we may choose for such an element $\left.\prod_{\varepsilon_{i}=-1}\left(e_{i}+f_{i}\right)\right)$.

We translate the action of $W(\operatorname{Cliff}(n, n))$ on the group of characters $L_{\mathrm{Cliff}(n, n)}$, provided with the basis $x_{0}, \ldots, x_{n}$ given by $\tau$. We get:

- For $\sigma \in \mathfrak{S}_{n}$ :

$$
\sigma x_{0}=x_{0}, \quad \sigma x_{i}=x_{\sigma^{-1}(i)}(i>0)
$$

- For $\varepsilon \in\{ \pm 1\}^{n}$ :

$$
\varepsilon x_{i}= \begin{cases}\varepsilon_{i} x_{i} & \text { if } i>0 \\ x_{0}+\sum_{j>0} \frac{1-\varepsilon_{j}}{2} x_{j} & \text { if } i=0 .\end{cases}
$$

On the other hand, a maximal torus of $\operatorname{Spin}(n, n)$ is given by $\operatorname{Ker}\left(\left(\gamma_{1}\right)_{\mid T}\right)$, hence $L_{\mathrm{Spin}(n, n)}$ is the quotient of $L_{\mathrm{Cliff}(n, n)}$ by the subgroup generated by

$$
\gamma_{1}=2 x_{0}+\sum_{i>0} x_{i}
$$

Let us also define

$$
\gamma_{2}=2 x_{0}^{2}+2 x_{0} \sum_{i>0} x_{i}+\sum_{0<i<j} x_{i} x_{j}
$$

The following proposition follows from elementary computations.

5.7. Proposition. We have

(i) $\left(L_{\mathrm{Cliff}(n, n)}\right)^{W}= \begin{cases}\mathbb{Z} \gamma_{1} & \text { for } n \geq 2, \\ \mathbb{Z} \gamma_{1} \oplus \mathbb{Z} x_{0} & \text { for } n=1 .\end{cases}$

(ii) $\left(L_{\operatorname{Spin}(n, n)}\right)^{W}= \begin{cases}0 & \text { for } n \geq 2, \\ \mathbb{Z} x_{0} & \text { for } n=1 .\end{cases}$

(iii) $\mathbf{S}^{2}\left(L_{\mathrm{Cliff}(n, n)}\right)^{W}= \begin{cases}\mathbb{Z} \gamma_{1}^{2} \oplus \mathbb{Z} \gamma_{2} & \text { for } n \geq 3, \\ \mathbb{Z} \gamma_{1}^{2} \oplus \mathbb{Z} \gamma_{2} \oplus \mathbb{Z} x_{0}\left(\gamma_{1}-x_{0}\right) & \text { for } n=2, \\ \mathbb{Z} \gamma_{1} x_{0} \oplus \mathbb{Z} \gamma_{1} x_{1} \oplus \mathbb{Z} x_{0}^{2} & \text { for } n=1 .\end{cases}$

(iv) $\mathbf{S}^{2}\left(L_{\operatorname{Spin}(n, n)}\right)^{W}= \begin{cases}\mathbb{Z} \gamma_{2} & \text { for } n \geq 3, \\ \mathbb{Z} \gamma_{2} \oplus \mathbb{Z} x_{0}^{2} & \text { for } n=2, \\ \mathbb{Z} x_{0}^{2} & \text { for } n=1 .\end{cases}$

(v) $\psi^{*} c_{2}=2 \gamma_{2}-\gamma_{1}^{2} \in \mathbf{S}^{2}\left(L_{\mathrm{Cliff}(n, n)}\right)$.

5.8. Theorem. For $n \geq 3$, restriction to the maximal torus yields isomorphisms:

$$
\begin{array}{ll}
H^{1}\left(B \text { Cliff }(n, n), \mathcal{K}_{1}\right)=\mathbb{Z} \gamma_{1}, & H^{1}\left(B \operatorname{Spin}(n, n), \mathcal{K}_{1}\right)=0, \\
H^{2}\left(B \text { Cliff }(n, n), \mathcal{K}_{2}\right)=\mathbb{Z} \gamma_{1}^{2} \oplus \mathbb{Z} \gamma_{2}, & H^{2}\left(B \operatorname{Spin}(n, n), \mathcal{K}_{2}\right)=\mathbb{Z} \gamma_{2} .
\end{array}
$$

We have the identity, valid for all $n \geq 1$ :

$$
\psi^{*} c_{2}=2 \gamma_{2}-\gamma_{1}^{2} \in H_{\text {Zar }}^{2}\left(B \operatorname{Cliff}(n, n), \mathcal{K}_{2}\right)
$$

where $\psi: \operatorname{Cliff}(n, n) \rightarrow S L(2 n)$ is the natural map.

5.9. Proposition. The Whitney formula holds for $\gamma_{1}$ and $\gamma_{2}$ : for $m, n \geq 1$ one has

$$
\begin{aligned}
& \rho^{*} \gamma_{1}=\gamma_{1} \times 1+1 \times \gamma_{1} \\
& \rho^{*} \gamma_{2}=\gamma_{2} \times 1+\gamma_{1} \times \gamma_{1}+1 \times \gamma_{2}
\end{aligned}
$$


where $\rho$ is the embedding $\operatorname{Cliff}(m, m) \times \operatorname{Cliff}(n, n) \hookrightarrow \operatorname{Cliff}(m+n, m+n) . \quad$ In particular, $\gamma_{1}$ and $\gamma_{2}$ are stable.

Proof. This is clear for $\gamma_{1}$. For $\gamma_{2}$, the easiest is to use formula (5.3) of Theorem 5.8. Note that $c_{2}$ is additive since it comes from $S L(2 n)$. So (5.3) gives the Whitney formula for $\gamma_{2}$ multiplied by 2 , and we can then divide by 2 since

$$
H_{\text {Zar }}^{2}\left(B(\operatorname{Cliff}(m, m) \times \operatorname{Cliff}(n, n)), \mathcal{K}_{2}\right)
$$

is torsion-free.

\section{Two invariants For Clifford Bundles}

In this section we associate to a torsor $F$ under $\operatorname{Cliff}(n, n)$ on a scheme $X$ two invariants with values in étale motivic cohomology of $X$, which are related to the second Chern class of the vector bundle underlying $F$. When $X=\operatorname{Spec} K, K$ a field, we relate these to the Arason invariant.

6.1. Recall Lichtenbaum's complexes $\Gamma(i)(i \leq 2)$ ([39], [40], [41]). One has $\Gamma(0)=$ $\mathbb{Z}$ placed in degree $0, \Gamma(1)=\mathbb{G}_{m}$ placed in degree 1 and $\Gamma(2)$ is constructed in [40]. There are products $\Gamma(i) \stackrel{L}{\otimes} \Gamma(j) \rightarrow \Gamma(i+j)$ for $i+j \leq 2$. If $X$ is a smooth variety defined over a field $k$, one has $([41],[33$, th. 1.1$])$ :

$$
\mathbb{H}_{\text {ét }}^{i}(X, \Gamma(2))= \begin{cases}0 & i \leq 0, \\ K_{3}(k(X))_{\text {ind }} & i=1, \\ H_{\text {Zar }}^{0}\left(X, \mathcal{K}_{2}\right) & i=2, \\ H_{\text {Zar }}^{1}\left(X, \mathcal{K}_{2}\right) & i=3,\end{cases}
$$

and an exact sequence

$$
0 \rightarrow C H^{2}(X) \rightarrow \mathbb{H}_{\text {ét }}^{4}(X, \Gamma(2)) \rightarrow H_{\text {Zar }}^{0}\left(X, \mathcal{H}^{3}(\mathbb{Q} / \mathbb{Z}(2))\right) \rightarrow 0
$$

when $\mathcal{H}^{j}(\mathcal{F})$ is the Zariski sheaf associated to the presheaf $U \mapsto H_{\text {ét }}^{j}(U, \mathcal{F})$.

This computation is done via the Leray spectral sequence for the map $\alpha: X_{\text {ét }} \rightarrow$ $X_{\mathrm{Zar}}$, together with the following computation of the Zariski sheaves:

$$
R^{q} \alpha_{*} \Gamma(2)= \begin{cases}0 & \text { for } q \leq 0, \\ \text { the constant sheaf } K_{3}(k(X))_{\text {ind }} & \text { for } q=1, \\ \mathcal{K}_{2} & \text { for } q=2, \\ 0 & \text { for } q=3, \\ \mathcal{H}^{q-1}(\mathbb{Q} / \mathbb{Z}(2)) & \text { for } q \geq 4 .\end{cases}
$$

Here the étale sheaf $\mathbb{Q} / \mathbb{Z}(2)$ is defined as $\lim _{\longrightarrow} \mu_{n}^{\otimes 2}$ if char $k=0$ and

$$
\underset{(n, \operatorname{char} F)=1}{\lim _{n}} \mu_{n}^{\otimes 2} \oplus \underset{r}{\lim _{r}} W_{r} \Omega_{\log }^{2}[-2],
$$

where $W_{r} \Omega_{\log }^{2}$ is the sheaf of logarithmic de Rham-Witt differentials over the big étale site of Spec $k$ and the transition maps are given by the Verlagerung (compare [33]).

On the other hand, one has ([41], [33, th. 1.2])

$$
\mathbb{H}_{\text {Zar }}^{i}(X, \Gamma(2))= \begin{cases}K_{3}(k(X))_{\text {ind }} & i=1, \\ H_{\text {Zar }}^{i-2}\left(X, \mathcal{K}_{2}\right) & 2 \leq i \leq 4, \\ 0 & \text { otherwise }\end{cases}
$$


6.2. Lemma. Let $X / k$ be a smooth, geometrically connected rational variety. (This means that $k_{s}(X) / k_{s}$ is a purely transcendental extension, where $k_{s}$ is a separable closure of $k$.) Then the map $K_{3}(k)_{\text {ind }} \rightarrow K_{3}(k(X))_{\text {ind }}$ is an isomorphism.

Proof. If $k(X) / k$ is purely transcendental, this follows from [37, pp. 327-328] or [48, lemma 4.2]. In general this follows from the commutative diagram

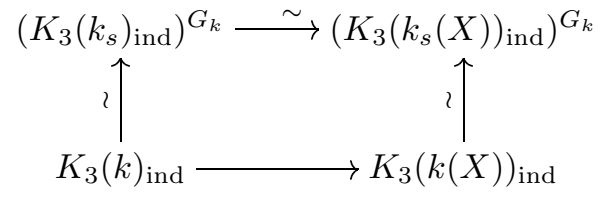

in which the vertical isomorphisms follow from [37, th. 4.13] or [48, prop. 11.4].

6.3. Remark. One could weaken the assumption "rational" into "unirational". It is in fact conjectured that the result holds for any geometrically connected $X$ (unirational or not) [48, Conj. 11.7].

Hypercohomology with coefficients in $\Gamma(2)$ extends to simplicial schemes. We have:

6.4. Lemma. a) Let $X$ • be a simplicial $k$-scheme, with all $X_{n}$ smooth, geometrically connected and rational (see Lemma 6.2). Then $\mathbb{H}_{\text {êt }}^{1}\left(X_{\bullet}, \Gamma(2)\right) \simeq K_{3}(k)_{\text {ind }}$ and the other formulce in (6.1) and (6.2) hold for étale and Zariski cohomology of $X$. (replacing $\mathrm{CH}^{2}(X)$ by $\mathbb{H}_{\text {Zar }}^{2}\left(X_{\bullet}, \mathcal{K}_{2}\right)$ in $(6.2)$ ).

b) Assume further that $X_{0}=\operatorname{Spec} k$. Then the exact sequence (6.2) degenerates into a canonical isomorphism

$$
\mathbb{H}_{\text {êt }}^{4}\left(X_{\bullet}, \Gamma(2)\right) \simeq H_{\text {êt }}^{3}(k, \mathbb{Q} / \mathbb{Z}(2)) \oplus \mathbb{H}_{\text {Zar }}^{2}\left(X_{\bullet}, \mathcal{K}_{2}\right) .
$$

This applies in particular to $X_{\bullet}=B G / k$, where $G$ is a connected linear algebraic group over $k$.

Proof. a) To compare $\mathbb{H}_{\text {êt }}^{*}\left(X_{\bullet}, \Gamma(2)\right)$ with $H_{\mathrm{Zar}}^{*}\left(X_{\bullet}, \mathcal{K}_{2}\right)$, we use the "Leray" spectral sequence

$$
E_{2}^{p, q}=H_{\text {Zar }}^{p}\left(X_{\bullet}, R^{q} \alpha_{*} \Gamma(2)\right) \Rightarrow \mathbb{H}_{\text {ét }}^{p+q}\left(X_{\bullet}, \Gamma(2)\right)
$$

where $\alpha$ is the natural map from the big étale site of Spec $k$ to its big Zariski site. The simplicial Zariski sheaves $R^{q} \alpha_{*} \Gamma(2)$ are given by (6.3); moreover the assumption on $X_{\bullet}$ and Lemma 6.2 imply that $R^{1} \alpha_{*} \Gamma(2)$ is the constant simplicial sheaf with value $K_{3}(k)_{\text {ind }}$. Therefore,

$$
E_{2}^{p, 1}=H_{\text {Zar }}^{p}\left(X_{\bullet}, K_{3}(k)_{\text {ind }}\right)= \begin{cases}K_{3}(k)_{\text {ind }} & p=0, \\ 0 & p>0,\end{cases}
$$

and the computations of [41], [33] apply mutatis mutandis. (For b), we observe that the composite map

$$
\begin{aligned}
H^{3}(k, \mathbb{Q} / \mathbb{Z}(2)) & =\mathbb{H}_{\text {ét }}^{4}(\operatorname{Spec}(k), \Gamma(2)) \rightarrow \mathbb{H}_{\text {êt }}^{4}\left(X_{\bullet}, \Gamma(2)\right) \rightarrow E_{2}^{0,4} \\
& =H_{\text {ét }}^{0}\left(X_{\bullet}, \mathcal{H}^{3}(\mathbb{Q} / \mathbb{Z}(2))\right)
\end{aligned}
$$

is bijective, which follows from the spectral sequence (4.1) and the assumptions on $X_{0}$. It follows that the differential $E_{2}^{0,4} \stackrel{d_{3}}{\rightarrow} E_{2}^{3,2}$ is 0 , even though the latter group may be nonzero.) The last claim follows from the fact that $G$, hence all $G^{p}$, are geometrically connected rational varieties over $k$. 
6.5. Let $X$ be a scheme. We shall give parallel definitions of Chern classes $c_{1}(V)$, $c_{2}(V)$ for a vector bundle $V$ on $X$ (with values in Zariski motivic cohomology) and classes $\gamma_{1}(F), \gamma_{2}(F)$ for a Clifford bundle $F$ on $X$ (with values in étale motivic cohomology). The classifying simplicial schemes $B G L(N)$ and $B \operatorname{Cliff}(n, n)$ used below will be considered over $\mathbb{Z}$.

6.6. Vector bundles. Let $V$ be a vector bundle of $\operatorname{rank} N$ on $X$. Then $V$ is locally trivial for the Zariski topology. Let $\left(U_{i}\right)$ be a Zariski cover of $X$ trivializing $V$, and let $U \bullet$ be the associated simplicial scheme. We have a diagram

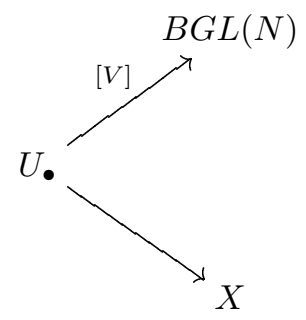

in which $X$ is considered as a constant simplicial scheme. The top map $[V]$ is induced by transition functions between given trivializations of $V$ on the $U_{i}$ 's. Since the bottom map induces an isomorphism on Zariski cohomology, (6.5) yields homomorphisms

$$
\mathbb{H}_{\text {Zar }}^{2 i}(B G L(N), \Gamma(i)) \stackrel{[V]^{*}}{\longrightarrow} \mathbb{H}_{\text {Zar }}^{2 i}(X, \Gamma(i))
$$

which only depend on the isomorphism class of $V$. We define

$$
\begin{aligned}
& c_{1}(V)=[V]^{*} c_{1} \in \mathbb{H}_{\text {Zar }}^{2}(X, \Gamma(1)), \\
& c_{2}(V)=[V]^{*} c_{2} \in \mathbb{H}_{\text {Zar }}^{4}(X, \Gamma(2)) .
\end{aligned}
$$

Note that $\mathbb{H}_{\mathrm{Zar}}^{2 i}(X, \Gamma(i))=H_{\mathrm{Zar}}^{i}\left(X, \mathcal{K}_{i}\right)(i \leq 2)$. If $X$ is a smooth variety over a field, then the Bloch-Quillen isomorphism

$$
H_{\text {Zar }}^{i}\left(X, \mathcal{K}_{i}\right) \simeq C H^{i}(X)
$$

together with Remark 5.4 identifies $c_{1}(V)$ and $c_{2}(V)$ with the classical Chern classes with values in the Chow ring of $X$.

6.7. Clifford bundles. Let $F$ be a torsor on $X$ under Cliff $(n, n)$ (briefly, a Cliff $(n, n)$-bundle). Then $F$ is locally trivial for the étale topology. Let $\left(U_{i}\right)$ be an étale cover of $X$ trivializing $F$, and let $U$ • be the associated simplicial scheme. We have a diagram

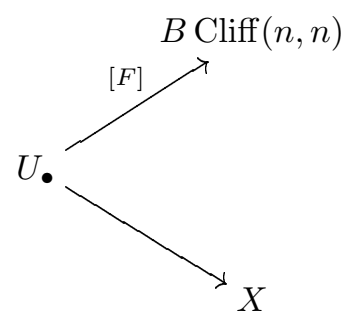

in which $X$ is considered as a constant simplicial scheme. The top map $[F]$ is induced by transition functions between given trivializations of $F$ on the $U_{i}$ 's. Since 
the bottom map induces an isomorphism on étale cohomology, (6.6) yields homomorphisms

$$
\mathbb{H}_{\text {êt }}^{2 i}(B \text { Cliff }(n, n), \Gamma(i)) \stackrel{[F]^{*}}{\longrightarrow} \mathbb{H}_{\text {ét }}^{2 i}(X, \Gamma(i))
$$

which only depend on the isomorphism class of $F$. We define

$$
\begin{aligned}
& \gamma_{1}(F)=[F]^{*} \gamma_{1} \in \mathbb{H}_{\text {ét }}^{2}(X, \Gamma(1)), \\
& \gamma_{2}(F)=[F]^{*} \gamma_{2} \in \mathbb{H}_{\text {ét }}^{4}(X, \Gamma(2)) .
\end{aligned}
$$

Note that, even though $\gamma_{1}$ and $\gamma_{2}$ are classes in $H_{\text {Zar }}^{i}\left(B \operatorname{Cliff}(n, n), \mathcal{K}_{i}\right)(i=$ $1,2),[F]$ is only defined in the étale topology, so $\gamma_{i}(F)$ is not a priori a Zariski cohomology class. The class $\gamma_{1}(F)$ certainly is, since $\mathbb{H}_{\text {Zar }}^{2}(X, \Gamma(1)) \rightarrow \mathbb{H}_{\text {ét }}^{2}(X, \Gamma(1))$ is an isomorphism. But, when $X$ is smooth over a field, the map

$$
C H^{2}(X) \simeq \mathbb{H}_{\text {Zar }}^{4}(X, \Gamma(2)) \rightarrow \mathbb{H}_{\text {ét }}^{4}(X, \Gamma(2))
$$

(cf. (6.4)) coincides with the map of (6.2). The main point of this paper is that in general $\gamma_{2}(F) \notin C H^{2}(X)$, i.e. is not algebraic.

6.8. By pushout, the map

$$
\operatorname{Cliff}(n, n) \rightarrow S O(n, n)
$$

associates to $F$ a $S O(n, n)$-torsor $E$ on $X$, that we shall call the underlying quadratic bundle of $F$. Similarly, the composite

$$
\operatorname{Cliff}(n, n) \rightarrow S O(n, n) \rightarrow S L(2 n)
$$

associates to $F$ an $S L(2 n)$-torsor $V$, the underlying vector bundle of $F$.

The vector bundle $V$ has a second Chern class $c_{2}(V) \in \mathbb{H}_{\text {Zar }}^{4}(X, \Gamma(2))$, that we may map to $\mathbb{H}_{\text {êt }}^{4}(X, \Gamma(2))$. If 2 is invertible on $X$, the quadratic bundle $E$ has a Clifford invariant $c(E) \in H_{\text {èt }}^{2}(X, \mathbb{Z} / 2)$ (Definition 2.3). We define $c_{2}(F)$ as $c_{2}(V) \in \mathbb{H}_{\text {êt }}^{4}(X, \Gamma(2))$ and $c(F)$ as $c(E)$.

6.9. Theorem. The characteristic classes

$$
\gamma_{1}(F) \in \mathbb{H}_{\text {êt }}^{2}(X, \Gamma(1)), \gamma_{2}(F) \in \mathbb{H}_{\text {ét }}^{4}(X, \Gamma(2))
$$

have the following properties and relations with $c_{2}(F) \in \mathbb{H}_{\text {êt }}^{4}(X, \Gamma(2))$ and $c(F) \in$ $H_{\text {ét }}^{2}(X, \mathbb{Z} / 2)\left(1 / 2 \in \mathcal{O}_{X}\right)$ :

(i) naturality for all morphisms;

(ii) additivity: $\left\{\begin{array}{l}\gamma_{1}\left(F \perp F^{\prime}\right)=\gamma_{1}(F)+\gamma_{1}\left(F^{\prime}\right), \\ \gamma_{2}\left(F \perp F^{\prime}\right)=\gamma_{2}(F)+\gamma_{1}(F) \cdot \gamma_{1}\left(F^{\prime}\right)+\gamma_{2}\left(F^{\prime}\right),\end{array}\right.$ where the product corresponds to the pairing $\Gamma(1) \times \Gamma(1) \rightarrow \Gamma(2)$;

(iii) relation with $c_{2}: c_{2}(F)=2 \gamma_{2}(F)-\gamma_{1}(F)^{2}$;

(iv) relation with $c\left(1 / 2 \in \mathcal{O}_{X}\right): \delta_{1}\left(\gamma_{1}(F)\right)=c(F)$, where $\delta_{1}: \mathbb{H}_{\text {ét }}^{2}(X, \Gamma(1))=$ $H_{\text {ét }}^{1}\left(X, \mathbb{G}_{m}\right) \rightarrow H_{\text {ét }}^{2}(X, \mathbb{Z} / 2)$ is the boundary map from the Kummer exact sequence.

Note that the class $\gamma_{1}(F)$ has an elementary description as the image of $[F] \in$ $H_{\text {ét }}^{1}(X, \operatorname{Cliff}(n, n))$ into $H_{\text {ét }}^{1}\left(X, \mathbb{G}_{m}\right)=\mathbb{H}_{\text {ét }}^{2}(X, \Gamma(1))$ via the spinor norm $\gamma_{1}$ of $(2.2)$.

Proof. (i) is trivial; (ii) and (iii) follow from Theorem 5.8. It remains to prove (iv). The diagram (2.2) gives an exact sequence

$$
1 \rightarrow \mu_{2} \rightarrow \operatorname{Cliff}(n, n) \stackrel{\left(\rho, \gamma_{1}\right)}{\longrightarrow} S O(n, n) \times \mathbb{G}_{m} \rightarrow 1
$$


and the induced composed map

$$
H^{1}(X, \operatorname{Cliff}(n, n)) \stackrel{\left(\rho_{*},\left(\gamma_{1}\right)_{*}\right)}{\longrightarrow} H^{1}(X, S O(n, n)) \times H^{1}\left(X, \mathbb{G}_{m}\right) \rightarrow H^{2}\left(X, \mu_{2}\right)
$$

of non-abelian cohomology pointed sets is zero. Hence the diagram

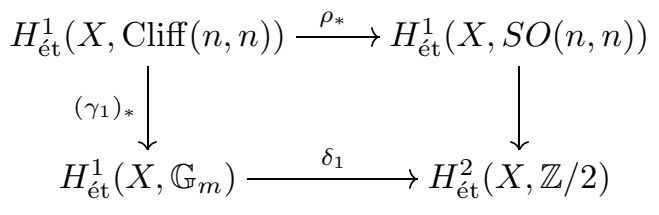

commutes.

Suppose $X=\operatorname{Spec} K$ where $K$ is a field of characteristic $\neq 2$. Then $\mathbb{H}^{2}(K, \Gamma(1))$ $=H^{1}\left(K, \mathbb{G}_{m}\right)=0$ (Hilbert 90) and $c_{2}(F)=0$ since any vector bundle over Spec $K$ is trivial. So $\gamma_{1}(F)=0$ and formula (ii) in Theorem 6.9 says that $\gamma_{2}$ is additive, while formula (iii) reduces to

$$
2 \gamma_{2}(E)=0
$$

6.10. Lemma. Let $F$ be a $\operatorname{Cliff}(n, n)$-bundle over a field $K$, and let $q$ be its underlying quadratic form. Then $q \in I^{3} K$.

Proof. By Merkurjev's theorem [43] it suffices to see that $c(q)=0$ : this follows immediately from Lemma 2.1 (or Theorem 6.9 (iv)).

6.11. Theorem. Let $F$ be a $\operatorname{Cliff}(n, n)$-bundle over $K$ and $q$ the underlying quadratic form. Then

$$
\delta_{2}\left(e^{3}(q)\right)=\gamma_{2}(F)
$$

where $\delta_{2}: H^{3}(K, \mathbb{Z} / 2) \rightarrow \mathbb{H}^{4}(K, \Gamma(2))$ is the boundary map coming from the Kummer triangle [41], [33, (9)]

$$
\mathbb{Z} / 2[-1] \rightarrow \Gamma(2) \stackrel{2}{\longrightarrow} \Gamma(2) \rightarrow \mathbb{Z} / 2
$$

Proof. Write $q+\sum \varphi_{i}=0$ in $W(K)$, where the $\varphi_{i}$ are multiples of 3-fold Pfister forms. So we have an isomorphism $q \perp \perp_{i} \varphi_{i} \cong m \mathbb{H}$ for some $m$. Letting $F_{i}$ and $H$ denote Cliff-bundles representing the $\varphi_{i}$ and $m \mathbb{H}$, we have (with obvious notation)

$$
F \perp \perp_{i} F_{i} \simeq H
$$

in view of Remark 2.2, hence $\gamma_{2}\left(F \perp \perp_{i} F_{i}\right)=\gamma_{2}(H)=0$, and by Theorem 6.9 (ii):

$$
\gamma_{2}(F)+\sum \gamma_{2}\left(F_{i}\right)=0
$$

(note that $\gamma_{1} \equiv 0$ on $\operatorname{Spec} k$ ). Since $e^{3}(q)=\sum e^{3}\left(\psi_{i}\right)$ too, we are reduced to the case in which $q$ is a 3 -fold Pfister form.

Recall that $\mathbb{H}^{3}(K, \Gamma(2))=0$ (see 6.1 ), so that $\delta_{2}$ is an isomorphism onto ${ }_{2} \mathbb{H}^{4}(K, \Gamma(2))$. By diagram (6.7), $\gamma_{2}(F)$ is 2-torsion: let $\widetilde{\gamma}_{2}(F)$ denote $\delta_{2}^{-1}\left(\gamma_{2}(F)\right) \in$ $H^{3}(K, \mathbb{Z} / 2)$.

Let $\widetilde{F}$ be a Spin-bundle lifting $F$, whose existence is assured by diagram (2.2) and Hilbert's Theorem 90. Then, by construction, $\widetilde{\gamma}_{2}(F)$ is nothing else than the Rost invariant associated with $\widetilde{F}$ (see Appendix B). Let $K_{1}=K(q)$ be the function field of the quadric defined by $q$ and $K_{2}=K(\widetilde{F})$ the function field of the torsor $\widetilde{F}$. Since $q$ is a Pfister form, $q_{K_{1}}$ is hyperbolic [35, cor. X.1.6], hence $\widetilde{F}_{K_{1}}$ is trivial as the map $H^{1}\left(K_{1}, \operatorname{Spin}(n, n)\right) \rightarrow H^{1}\left(K_{1}, S O(n, n)\right)$ has trivial kernel (this follows 
from the surjectivity of the spinor norm for isotropic forms). Conversely, $\widetilde{F}_{K_{2}}$ is trivial, hence $q_{K_{2}}$ is hyperbolic. It follows that

$$
e^{3}(q), \widetilde{\gamma}_{2}(F) \in \operatorname{Ker}\left(H^{3}(K, \mathbb{Z} / 2) \rightarrow H^{3}\left(K_{1}, \mathbb{Z} / 2\right)\right)
$$

$$
\cap \operatorname{Ker}\left(H^{3}(K, \mathbb{Z} / 2) \rightarrow H^{3}\left(K_{2}, \mathbb{Z} / 2\right)\right) .
$$

By Arason's theorem [1, th. 5.6], the first kernel is generated by $e^{3}(q)$. By Rost's theorem (Theorem B.11), the second kernel is generated by $\widetilde{\gamma}_{2}(F)$. Therefore $\widetilde{\gamma}_{2}(F)=e^{3}(q)$, as we wanted.

\section{Snaking a Bloch-Ogus differential}

Let $X$ be a smooth variety over $k$ and $n$ be prime to char $k$. Consider the commutative diagram with exact rows and columns:

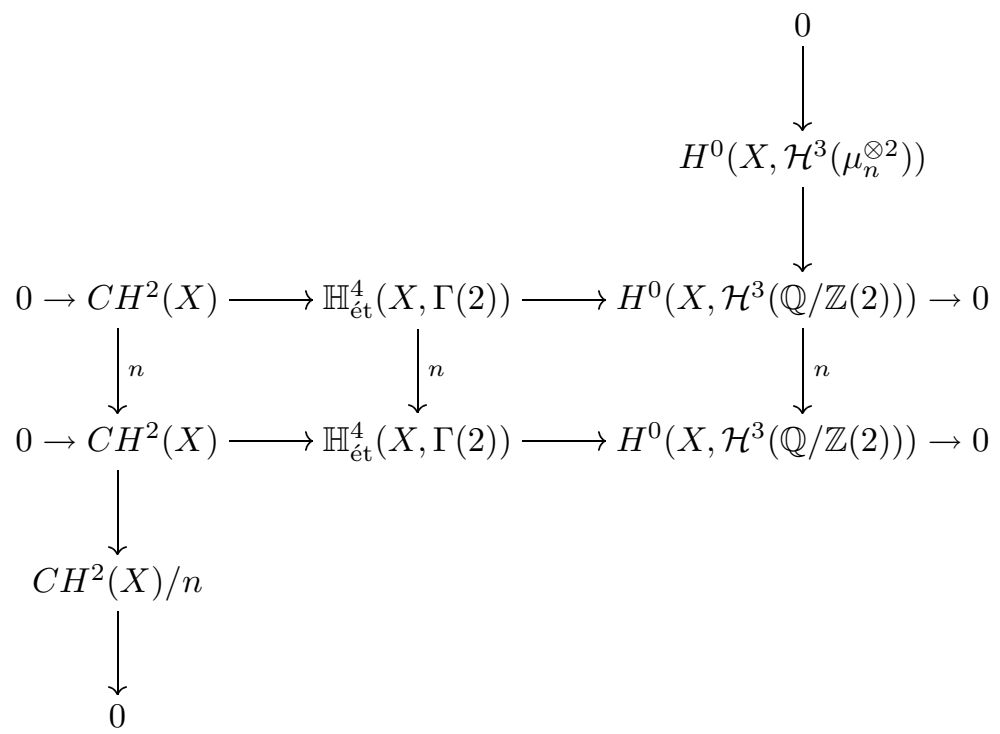

The snake lemma defines a map

$$
\begin{gathered}
\mathcal{S}: H^{0}\left(X, \mathcal{H}^{3}\left(\mu_{n}^{\otimes 2}\right)\right) \rightarrow C H^{2}(X) / n, \\
\mathcal{S}(x)=\operatorname{Im} n \widetilde{x} \in C H^{2}(X) / n
\end{gathered}
$$

where $\widetilde{x}$ is a lift of $x$ in $\mathbb{H}_{\text {ét }}^{4}(X, \Gamma(2))$.

\subsection{Theorem. Let}

$$
d_{2}: H^{0}\left(X, \mathcal{H}^{3}\left(\mu_{n}^{\otimes 2}\right)\right) \rightarrow H^{2}\left(X, \mathcal{H}^{2}\left(\mu_{n}^{\otimes 2}\right)\right) \simeq C H^{2}(X) / n
$$

be the $d_{2}$-differential from the Bloch-Ogus spectral sequence. Then $d_{2}=\mathcal{S}$.

Proof. Let $I^{*}$ be a torsion free acyclic complex quasi-isomorphic to $\Gamma(2)$. Multiplication by $n$ gives an injective map of complexes:

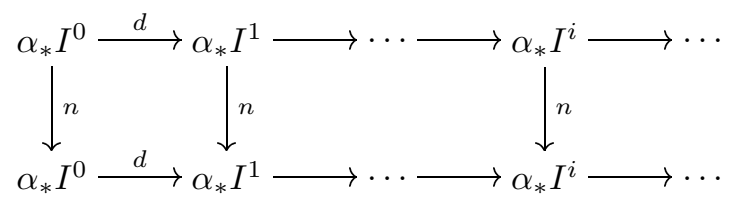


with cokernel $\alpha_{*} I^{i} / n \alpha_{*} I^{i}=\alpha_{*}\left(I^{i} / n I^{i}\right)$ quasi-isomorphic to $\mu_{n}^{\otimes 2}$.

Any $n$-torsion class $e \in H^{0}\left(X, R^{4} \alpha_{*} \Gamma(2)\right)=H^{0}\left(X, \mathcal{H}^{3}(\mathbb{Q} / \mathbb{Z}(2))\right)$ is represented on a suitable Zariski covering $X_{\bullet}$ of $X$ by $x_{i} \in \Gamma\left(X_{i}, \alpha_{*} I^{4}\right)_{d}$ closed, with $d x_{i}=0$, $n x_{i}=d y_{i}$ for some $y_{i} \in \Gamma\left(X_{i}, \alpha_{*} I^{3}\right)$. To obtain $\mathcal{S}(e)$, one first lifts $e$ as a class in $\mathbb{H}_{\text {êt }}^{4}(X, \Gamma(2))$, with Čech cocycle

$$
x=\left(x_{i_{0} \ldots i_{4}}, x_{i_{0} \ldots i_{3}}, \ldots, x_{i_{0}}\right) \in\left(\mathcal{C}^{4}\left(\alpha_{*} I^{0}\right) \times \ldots \times \mathcal{C}^{0}\left(\alpha_{*} I^{4}\right)\right)_{d-\delta},
$$

where $\delta$ is the Čech differential. Thus

$$
\begin{aligned}
n x & =\left(n x_{i_{0} \ldots i_{4}}, \ldots, n x_{i_{0}}\right) \\
& \equiv\left(n x_{i_{0} \ldots i_{4}}, \ldots, n x_{i_{0} i_{1}}-(\delta y)_{i_{0}, i_{1}}, 0\right) .
\end{aligned}
$$

As $R^{3} \alpha_{*} \Gamma(2)=0$, there are (after refining $\left.X_{\bullet}\right)$ elements $z_{i_{0} i_{1}} \in \mathcal{C}^{1}\left(\alpha_{*} I^{2}\right)$ verifying: $d z_{i_{0} i_{1}}=n x_{i_{0} i_{1}}-(\delta y)_{i_{0} i_{1}}$. Thus

$$
n x \equiv\left(n x_{i_{0} \ldots i_{4}}, n x_{i_{0} i_{1} i_{2} i_{3}}, n x_{i_{0} i_{1} i_{2}}-(\delta z)_{i_{0} i_{1} i_{2}}, 0,0\right)
$$

and $\mathcal{S}(e)$ is the class of

$$
n x_{i_{0} i_{1} i_{2}}-(\delta z)_{i_{0} i_{1} i_{2}} \text { in } C H^{2}(X)=H_{\text {Zar }}^{2}\left(X, R^{2} \alpha_{*} \Gamma(2)\right) .
$$

On the other hand, $d_{2}(e)$ is obtained as follows:

$$
e \text { as a class in } H_{\mathrm{Zar}}^{0}\left(X, \frac{\operatorname{Ker}\left\{\alpha_{*}\left(I^{3} / n\right) \rightarrow \alpha_{*}\left(I^{4} / n\right)\right\}}{\operatorname{Im} \alpha_{*}\left(I^{2} / n\right)}\right)
$$

is given by $y_{i}(\bmod n)$.

One takes $y_{i_{0} i_{1}} \in \Gamma\left(X_{i_{0} i_{1}}, \alpha_{*} I^{2} / n\right)$ verifying $d y_{i_{0} i_{1}}=(\delta y)_{i_{0} i_{1}} \bmod n$.

Then $d_{2}(e)=\delta y \in H_{\mathrm{Zar}}^{2}\left(X, \mathcal{H}^{2}\left(\mu_{n}^{\otimes 2}\right)\right)$. But we can take $y_{i_{0} i_{1}} \equiv-z_{i_{0} i_{1}}(\bmod n)$. Thus

$$
\delta y \equiv-\delta z \quad(\bmod n) \equiv+n x-\delta z \quad(\bmod n) .
$$

This is $\mathcal{S}(e)$.

\section{Proof of Theorem 1}

First we remark that $e=e^{3}(q)$ lies in $H^{0}\left(X, \mathcal{H}^{3}(\mathbb{Z} / 2)\right)$ : this is obvious from Theorem 6.11 and the fact that $H_{\text {ét }}^{3}(\mathcal{O}, \mathbb{Z} / 2) \stackrel{\delta_{2}}{\rightarrow}{ }_{2} \mathbb{H}_{\text {ét }}^{4}(\mathcal{O}, \Gamma(2))$ is bijective for the local rings $\mathcal{O}$ of $X$ (Hilbert 90 for $K_{2}$, see (6.3) and (6.8)).

We now consider the signed discriminant and Clifford invariant $d_{ \pm} E, c(E)$ of Definition 2.3. The Bloch-Ogus spectral sequence gives an exact sequence

$$
0 \rightarrow \operatorname{Pic}(X) / 2 \rightarrow H_{\text {ét }}^{2}(X, \mathbb{Z} / 2) \rightarrow H^{2}(K, \mathbb{Z} / 2) .
$$

8.1. Lemma. We have

$$
\begin{gathered}
d_{ \pm} E=0, \\
{[E] \in \operatorname{Im}\left(H_{\text {ét }}^{1}(X, S O(n, n))\right) \rightarrow H_{\text {ét }}^{1}(X, O(n, n)),{ }^{1}}
\end{gathered}
$$

and

$$
c(E) \in \operatorname{Pic}(X) / 2 \text {. }
$$

${ }^{1}$ Although $H_{\text {ét }}^{1}(X, S O(n, n)) \rightarrow H_{\text {ét }}^{1}(X, O(n, n))$ is injective if $X$ is the spectrum of a field, it need not be in general: we are indebted to Serre and Parimala for pointing this out. 
Proof. By assumption we have

$$
\left(d_{ \pm} E\right)_{\eta}=0=c(E)_{\eta}
$$

where $\eta$ is the generic point of $X$. Since $H_{\text {ét }}^{1}(X, \mathbb{Z} / 2) \rightarrow H^{1}(K, \mathbb{Z} / 2)$ is injective, this gives the first two claims, and the third follows from (8.1).

8.2. Lemma. The class $[E] \in H_{\text {êt }}^{1}(X, O(n, n))$ is in the image of

$$
H_{\text {ét }}^{1}(X, \operatorname{Cliff}(n, n)) \rightarrow H_{\text {ét }}^{1}(X, O(n, n)) .
$$

Proof. By Lemma 8.1, $[E]$ can be lifted to $H_{\text {ét }}^{1}(X, S O(n, n))$. Diagram (2.2) gives a commutative diagram of pointed sets

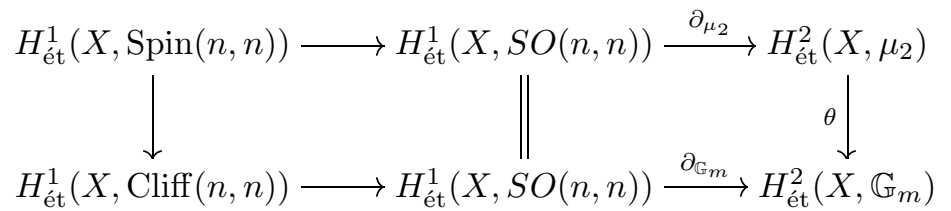

But by the Kummer exact sequence, $\operatorname{Pic}(X) / 2=\operatorname{Ker} \theta$, so

$$
\partial_{\mathbb{G}_{m}}([E])=\theta\left(\partial_{\mu_{2}}([E])\right)=\theta(c(E))=0
$$

by Lemma 8.1 .

Let $F$ be a Cliff-bundle refining $E$ (Lemma 8.2). By Theorem 6.11 , we have

$$
\gamma_{2}(F)_{\eta}=\delta\left(e^{3}(q)\right)
$$

where $\delta$ is the "Kummer" boundary for weight-two étale motivic cohomology. By Theorem 7.1, we have

$$
d_{2}\left(e^{3}(q)\right)=\mathcal{S}\left(e^{3}(q)\right)
$$

where $\mathcal{S}$ is the snake map of section 7. Equation (8.2) and the commutative diagram

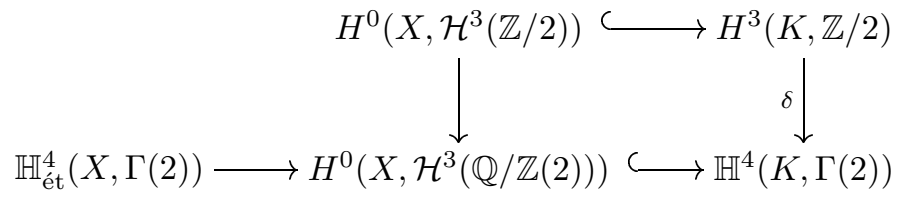

shows that $\gamma_{2}(F)$ lifts the image of $e^{3}(q)$ in $H^{0}\left(X, \mathcal{H}^{3}(\mathbb{Q} / \mathbb{Z}(2))\right)$. Therefore, the image of $e^{3}(q)$ by $\mathcal{S}$ is the projection of $2 \gamma_{2}(F) \in C H^{2}(X)$ in $C H^{2}(X) / 2$. By Theorem 6.9 (iii), this is $c_{2}(E)+\gamma_{1}(F)^{2}$. But by Theorem 6.9 (iv) we have $\delta_{1}\left(\gamma_{1}(F)\right)=c(F)$, hence (with an obvious abuse of notation) $\gamma_{1}(F)=c(E) \in \operatorname{Pic}(X) / 2$. Theorem 1 is proven.

\section{Application to QUADRATIC FORMS}

Let $q$ be a quadratic form over $k$. We assume $q \in I^{2} k$, i.e. $\operatorname{dim} q$ even and $d_{ \pm} q=1$. The Clifford algebra $C(q)$ is central simple over $k$ : let $X$ be its SeveriBrauer variety and $K=k(X)$. Over $K, C(q)$ is split, hence (by Merkurjev's and Arason's theorems) $q_{K} \in I^{3} K$ and $e^{3}(q)$ is defined.

9.1. Theorem. If ind $C(q) \geq 8$, then $d_{2}\left(e^{3}\left(q_{K}\right)\right) \neq 0$.

9.2. Corollary. Under the conditions of Theorem 9.1, $e^{3}\left(q_{K}\right) \neq 0$, hence $q_{K} \notin$ $I^{4} K$. 
9.3. Corollary. Let $n, i \geq 0$ and let $Q(k, 2 n, i)$ be the set of isomorphism classes of quadratic forms $q$ over $k$ such that $\operatorname{dim} q=2 n, d_{ \pm} q=1$ and ind $C(q) \leq i$. Then, if $i \geq 8$, there exists no cohomological invariant $e_{F}: Q(F, 2 n, i) \rightarrow H^{3}(F, \mathbb{Z} / 2)$ $(F \supseteq k)$ commuting with change of base field and such that $e(q)=e^{3}(q)$ if $q \in I^{3} F$.

Corollary 9.2 is wrong if ind $C(q) \leq 4$. For example, let $q$ be a non-hyperbolic Albert form; then $q_{K} \sim 0$. Similarly, Corollary 9.3 is wrong for $i=2$ : one can then define $e(q)=e^{3}(q \perp \tau)$, where $\tau$ is the quaternion form such that $c(q)=c(\tau)$. On the other hand, it seems likely that Corollary 9.3 still holds if $i=4$, provided $n \geq 4$.

Proof of Theorem 9.1. Let $E$ be the quadratic bundle $q \otimes_{k} X$, with generic fiber $q_{K}$ : we are in the situation of Theorem 1 . Since $E$ is extended from $k$, its underlying vector bundle is trivial, hence $c_{2}(E)=0$ and Theorem 1 reduces to

$$
d_{2} e^{3}\left(q_{K}\right)=c(E)^{2} \text {. }
$$

To prove Theorem 9.1, it therefore suffices to show that $c(E)^{2} \neq 0 \in C H^{2}(X) / 2$. Let $\bar{X}=X \otimes_{k} k_{s}$, where $k_{s}$ is a separable closure of $k$. Recall that $\bar{X} \simeq \mathbb{P}_{k_{s}}^{n}$, with $n=\operatorname{dim} X$. On the other hand, we have:

9.4. Lemma. Let $A$ be a central simple algebra of exponent 2 over $k$, and let $X$ be its Severi-Brauer variety.

a) Suppose that ind $A>1$. Then the $\operatorname{map} \operatorname{Pic}(X) \rightarrow \operatorname{Pic}(\bar{X})$ is injective and its image is $2 \mathbb{Z} h$, where $h$ is the class of a hyperplane section in $\operatorname{Pic}(X)$.

b) Suppose that ind $A \geq 8$. Then $\operatorname{Im}\left(C H^{2}(X) \rightarrow C H^{2}(\bar{X})\right)=4 \mathbb{Z} h^{2}$.

In particular, if $H=2 h$ is the generator of $\operatorname{Pic}(X)$, then $H^{2} \notin 2 C H^{2}(X)$.

Proof. Recall first that $C H^{i}\left(\mathbb{P}_{k_{s}}^{n}\right)=h^{i} \mathbb{Z}$, where $h$ is the class of a hyperplane section.

a) The injectivity is well-known and the value of the image is an easy consequence of Roquette's results on the Brauer group [55] (see also Panin [50]). (One can also get it from the calculation below.)

b) From a), we evidently have the inclusion $\supseteq$. To get the reverse inclusion, note the commutative diagram

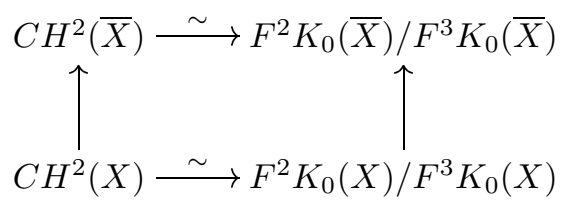

where $F^{i} K_{0}$ is the topological filtration on $K_{0}$. By Quillen's theorem [54, th. 8.4.1], $K_{0}(X)$ can be identified with the subgroup of $K_{0}(\bar{X})=K_{0}\left(\mathbb{P}^{n}\right)$ generated by elements of the form $\operatorname{ind}\left(A^{\otimes i}\right)(1+h)^{i}, i \geq 0$. Since $A$ has exponent 2 , these polynomials are

$$
\begin{aligned}
& p_{i}=(1+h)^{2 i} \quad=1+2 i h+(2 i-1) i h^{2}+\ldots \quad(i \geq 0), \\
& q_{i}=a(1+h)^{2 i+1}=a+(2 i+1) a h+(2 i+1) i a h^{2}+\ldots \quad(i \geq 0)
\end{aligned}
$$

where $a=\operatorname{ind} A$.

On the other hand, the group $F^{q} K_{0}(\bar{X})$ is the subgroup of $K_{0}(\bar{X})$ formed of those polynomials $\sum a_{n} h^{n}\left(a_{n} \in \mathbb{Z}\right)$ such that $a_{0}=\cdots=a_{q-1}=0$, and $F^{q} K_{0}(X)=$ 
$K_{0}(X) \cap F^{q} K_{0}(\bar{X})$. Let $\lambda_{i}, \mu_{i} \in \mathbb{Z}$ be such that $p=\sum_{i} \lambda_{i} p_{i}+\mu_{i} q_{i} \in F^{2} K_{0}(X)$. This means that

$$
\begin{aligned}
\sum_{i} \lambda_{i}+a \sum_{i} \mu_{i} & =0, \\
\sum_{i} 2 i \lambda_{i}+a \sum(2 i+1) \lambda_{i} & =0 .
\end{aligned}
$$

Since by assumption $a$ is divisible by 8 , the second equality implies

$$
\sum_{i} i \lambda_{i} \equiv 0 \quad(\bmod 4)
$$

The coefficient of $h^{2}$ in $p$ is now

$$
\sum_{i}(2 i-1) i \lambda_{i}+a \sum_{i}(2 i+1) i \mu_{i} \equiv \sum 2 i^{2} \lambda_{i}-\sum_{i} i \lambda_{i} \equiv 0 \quad(\bmod 4)
$$

by the above, since $i^{2} \equiv i(\bmod 2)$.

By the Hochschild-Serre spectral sequence, $H^{2}(k, \mathbb{Z} / 2) \rightarrow H^{2}(X, \mathbb{Z} / 2)$ is injective; in particular $0 \neq c(E) \in \operatorname{Pic}(X) / 2 \subseteq H^{2}(X, \mathbb{Z} / 2)$. It follows from this and Lemma 9.4 that $\operatorname{Pic}(X) / 2 \simeq \mathbb{Z} / 2$ and $c(E)$ generates $\operatorname{Pic}(X) / 2$, hence $c(E) \equiv H$ $(\bmod 2 \operatorname{Pic}(X))$. Therefore $c(E)^{2} \equiv H^{2} \not \equiv 0\left(\bmod 2 C H^{2}(X)\right)$.

Proof of Corollary 9.3. Suppose $e$ exists. Let $q \in Q(k, n, i)$ with ind $C(q) \geq 8$. Let $K$ be the function field of the Severi-Brauer variety of $C(q)$. By naturality of $e$, $e(q)_{K}=e\left(q_{K}\right)=e^{3}\left(q_{K}\right)$. Then $e^{3}\left(q_{K}\right)$ is defined over $k$, hence $d_{2}\left(e^{3}\left(q_{K}\right)\right)=0$, which contradicts Theorem 9.1.

9.5. Example. $\operatorname{dim} q=8$. Then $q_{K}$ is similar to a 3 -fold Pfister form. If ind $C(q)$ $=8$, Corollary 9.2 implies that $q_{K}$ is not hyperbolic, hence anisotropic. Laghribi [34] has shown that this still holds if ind $C(q)<8$, but the reason is entirely different: it relies on the Arason-Pfister Hauptsatz.

\section{Appendix A. Toral Descent}

A.1. Let $\pi: X \rightarrow Y$ be a morphism of schemes, and let $X_{Y}^{n}$ denote the $n$-fold fiber product of $X$ over $Y$. Form the simplicial scheme $E_{Y} X$ with $n$-simplices $X_{Y}^{n+1}$, where the map

$$
E_{Y} X(g): X_{Y}^{m+1} \rightarrow X_{Y}^{n+1}
$$

coming from $g: \Delta_{n} \rightarrow \Delta_{m}$ in $\Delta$ is given on ring-valued points by

$$
E_{Y} X(g)\left(x_{0}, \ldots, x_{m}\right)=\left(x_{g(0)}, \ldots, x_{g(n)}\right) .
$$

If we are working in the category of schemes over a fixed base $B$, we write $E X$ for $E_{B} X$.

The map $\pi$ induces a natural augmentation $\varepsilon_{X / Y}: E_{Y} X \rightarrow Y$.

The construction of $E_{Y} X$ is functorial in the map $X \rightarrow Y$; in particular, if $X \rightarrow Y$ is a map of simplicial schemes, we have the bi-simplicial scheme $E_{Y} X$, with $(n, m)$-simplices given by

$$
\left(E_{Y} X\right)_{(n, m)}=\left(E_{Y_{m}} X_{m}\right)_{n}
$$

and with augmentation $\varepsilon_{X / Y}: E_{X} Y \rightarrow Y$. 
Let $\mathcal{F}$ be a sheaf over the big Zariski site of $k$. The augmentation $\varepsilon_{X / Y}$ gives a natural map

$$
\varepsilon_{X / Y}^{*}: H^{*}(Y, \mathcal{F}) \rightarrow H^{*}\left(E_{Y} X, \mathcal{F}\right)
$$

A.2. Lemma. Let $X \stackrel{\pi}{\rightarrow} Y$ be a map of (simplicial) schemes. Suppose $\pi$ has a section $\sigma$. Then the augmentation map $E_{Y} X \stackrel{\varepsilon_{X / Y}}{\longrightarrow} Y$ is a homotopy equivalence, where we consider $Y$ as a constant (bi)-simplicial scheme, and (A.1) is an isomorphism. In particular, if $\mathcal{C}$ is a subcategory of the category of $k$-schemes, closed under finite products over $k$, and if $\mathcal{F}$ is a Zariski sheaf on $\mathcal{C}$, then the map

$$
\varepsilon_{X / k}^{*}: H^{0}(\operatorname{Spec}(k), \mathcal{F})=H^{*}(\operatorname{Spec}(k), \mathcal{F}) \rightarrow H^{*}(E X, \mathcal{F})
$$

is an isomorphism for all $X$ in $\mathcal{C}$ having a $k$-point.

Proof. For notational simplicity, we give the proof supposing that $X$ and $Y$ are schemes. The section $\sigma$ induces a map $E \sigma: E_{Y} Y=Y \rightarrow E_{Y} X$ splitting $\varepsilon_{X / Y}$.

The simplicial set $[0,1]$ is the nerve of the category associated to the partially ordered set $0<1$, hence $[0,1]$ has $n$-simplices given as the set of length $n+1$ nondecreasing sequences of 0 's and 1's. Given such a sequence $s:\{0, \ldots, n\} \rightarrow\{0,1\}$, define

$$
p_{s}: X_{Y}^{n+1} \rightarrow X_{Y}^{n+1}
$$

by

$$
p_{s}\left(x_{0}, \ldots, x_{n}\right)=\left(y_{0}, \ldots, y_{n}\right)
$$

where $y_{i}=\sigma\left(\pi\left(x_{i}\right)\right)$ if $s(i)=0$ and $y_{i}=x_{i}$ if $s(i)=1$. Letting $E_{Y} X \times[0,1]$ be the diagonal simplicial scheme

$$
\left(E_{Y} X \times[0,1]\right)_{n}:=\left(E_{Y} X\right)_{n} \times[0,1]_{n}
$$

the maps $p_{s}$ define the map of simplicial schemes

$$
p: E_{Y} X \times[0,1] \rightarrow E_{Y} X
$$

with

$$
p_{\mid E_{Y} X \times 0}=E \sigma \circ \varepsilon_{X / Y} ; \quad p_{\mid E_{Y} X \times 1}=\operatorname{id}_{E_{Y} X} .
$$

Let $X$ be a simplicial scheme, $T \cong \mathbb{G}_{m}^{r}$ a split torus of rank $r$, and $\mu: X \times T \rightarrow X$ an action of $T$ on $X$. We call the $T$-action free if the action on the $n$-simplices $X_{n} \times T \rightarrow X_{n}$ is free for each $n$. Assuming that the quotients $X_{n} / T$ exist for each $n$, we may form the simplicial scheme $Y$ with $n$-simplices $Y_{n}:=X_{n} / T$ and canonical morphism $\pi: X \rightarrow Y$.

A.3. Proposition. Let $\mu: X \times T \rightarrow X$ be a free $T$-action on a smooth (simplicial) $k$-scheme $X$ such that the quotient $X \rightarrow Y:=X / T$ exists. Then the map (A.1) is an isomorphism.

Proof. Suppose that $X$ is a smooth simplicial $k$-scheme. We have the spectral sequences

$$
\begin{gathered}
E_{1}^{p, q}\left(E_{Y} X\right)=H^{q}\left(E_{Y_{p}} X_{p}, \mathcal{F}\right) \Longrightarrow H^{p+q}\left(E_{Y} X, \mathcal{F}\right), \\
E_{1}^{p, q}(Y)=H^{q}\left(Y_{p}, \mathcal{F}\right) \Longrightarrow H^{p+q}(Y, \mathcal{F})
\end{gathered}
$$

and the augmentation induces a map of spectral sequences. This reduces us to considering the case of a smooth $k$-scheme $X$ with $T$-action. 
Since the $T$-action is free, the map $X \rightarrow Y:=X / T$ makes $X$ into a $T$-torsor for the étale topology. By Hilbert's Theorem $90, X \rightarrow Y$ is a Zariski locally trivial $T$-bundle. Let

$$
\mathcal{U}:=\left\{U_{0}, \ldots, U_{s}\right\}
$$

be a Zariski open cover of $Y$ trivializing $\pi: X \rightarrow Y$, let $V_{i}=\pi^{-1}\left(U_{i}\right)$, and let $\mathcal{V}$ be the open cover $\left\{V_{0}, \ldots, V_{s}\right\}$ of $X$. We form the simplicial schemes $\mathcal{N}(\mathcal{U})$ and $\mathcal{N}(\mathcal{V})$ (where $\mathcal{N}$ stands for "nerve"), giving the map of augmented simplicial schemes

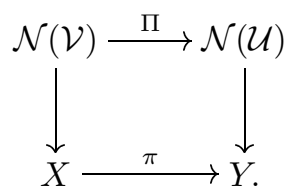

This induces the augmentation

$$
\varepsilon_{\Pi}: E_{\mathcal{N}(\mathcal{U})} \mathcal{N}(\mathcal{V}) \rightarrow \mathcal{N}(\mathcal{U})
$$

and the commutative diagram

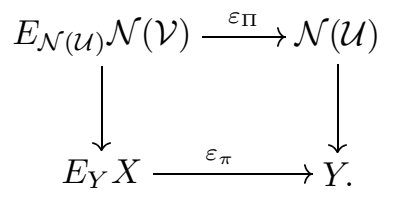

As Zariski cohomology of a Zariski sheaf satisfies Mayer-Vietoris for Zariski open covers, the right-hand vertical arrow in (A.2) induces an isomorphism

$$
H^{*}(Y, \mathcal{F}) \rightarrow H^{*}(\mathcal{N}(\mathcal{U}), \mathcal{F})
$$

The cover $\mathcal{V}$ induces a cover $\mathcal{V}_{n}$ of $\left(E_{Y} X\right)_{n}$ by the open subsets $\left\{\left(E_{U_{0}} V_{0}\right)_{n}, \ldots\right.$, $\left.\left(E_{U_{s}} V_{s}\right)_{n}\right\}$. We have the canonical identification

$$
\left[E_{\mathcal{N}(\mathcal{U})} \mathcal{N}(\mathcal{V})\right]_{n, *} \cong \mathcal{N}\left(\mathcal{V}_{n}\right)
$$

By a spectral sequence argument as above, this implies that the left-hand vertical arrow in (A.2) induces an isomorphism

$$
H^{*}\left(E_{Y} X, \mathcal{F}\right) \rightarrow H^{*}\left(E_{\mathcal{N}(\mathcal{U})} \mathcal{N}(\mathcal{V}), \mathcal{F}\right)
$$

Using the other spectral sequence for the cohomology of $E_{\mathcal{N}(\mathcal{U})} \mathcal{N}(\mathcal{V})$ and $\mathcal{N}(\mathcal{U})$, we thus reduce to the case $X=Y \times_{k} T$, with $T$ acting by multiplication on the factor $T$.

In this case, the projection $X \rightarrow Y$ has the section $\sigma: Y \rightarrow X$ given by $\sigma(y)=$ $(y, 1)$. We then apply Lemma A.2.

A.4. Remark. The proof works just as well for $X \rightarrow Y$ a Zariski-locally trivial family with fiber $F$, such that $F$ has a $k$-point, and similarly for $X \rightarrow Y$ an étalelocally trivial family with fiber $F$, such that $F$ has a $k$-point, provided we use étale cohomology instead of Zariski cohomology.

A.5. Proposition. Let $f: X \rightarrow X^{\prime}$ be a T-equivariant map of smooth (simplicial) $k$-schemes with free $T$-action, such that the quotients $Y:=X / T$ and $Y^{\prime}:=X^{\prime} / T$ are defined, and let $g: Y \rightarrow Y^{\prime}$ be the induced map. Suppose that $f$ induces an isomorphism

$$
f^{*}: H^{*}\left(X^{\prime}, \mathcal{F}\right) \rightarrow H^{*}(X, \mathcal{F})
$$


for all sheaves $\mathcal{F}$ on the big Zariski site over $k$. Then $g$ induces an isomorphism

$$
g^{*}: H^{*}\left(Y^{\prime}, \mathcal{F}\right) \rightarrow H^{*}(Y, \mathcal{F})
$$

for all sheaves $\mathcal{F}$.

Proof. The commutative diagram

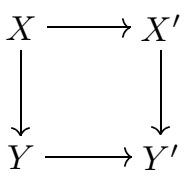

defines a commutative diagram

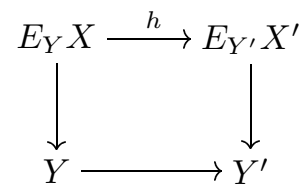

By Proposition A.3, we need only show that the map

$$
h^{*}: H^{*}\left(E_{Y^{\prime}} X^{\prime}, \mathcal{F}\right) \rightarrow H^{*}\left(E_{Y} X, \mathcal{F}\right)
$$

is an isomorphism. The map $h$ induces a map of spectral sequences, given on the $E_{1}$-terms by

$$
E_{1}^{p, q}\left(E_{Y^{\prime}} X^{\prime}\right)=H^{q}\left(\left(E_{Y^{\prime}} X^{\prime}\right)_{p, *}, \mathcal{F}\right) \stackrel{h_{p}^{*}}{\longrightarrow} E_{1}^{p, q}\left(E_{Y} X\right)=H^{q}\left(\left(E_{Y} X\right)_{p, *}, \mathcal{F}\right) .
$$

We have natural isomorphisms

$$
\left(E_{Y} X\right)_{p, *} \cong X_{*} \times_{k} T^{p} ; \quad\left(E_{Y^{\prime}} X^{\prime}\right)_{p, *} \cong X_{*}^{\prime} \times_{k} T^{p}
$$

which identify the map $h_{p}$ on $p$-simplices with $f \times \mathrm{id}_{T^{p}}$. The cohomology of each of these spaces is the abutment of Leray spectral sequences

$$
E_{2}^{p, q}=H^{p}\left(X, R^{q} \pi_{*} \mathcal{F}\right) \Rightarrow H^{p+q}\left(X \times_{k} T^{p}, \mathcal{F}\right)
$$

(where $\pi: X \times_{k} T^{p} \rightarrow X$ is the first projection) and similarly for $X^{\prime}$. By our assumption on $f$ and a spectral sequence comparison argument, each $h_{p}^{*}$ is an isomorphism.

A.6. Example. Let $T$ be a split torus in a reductive algebraic group scheme $G$. Then the diagonal action of $T$ on $G^{n}$ and on $T^{n}$ is free, giving the $T$-equivariant morphism $E T \rightarrow E G$ induced by the inclusion of $T$ into $G$. It is easy to see that the quotient $E G / T$ exists; the quotient $E T / T$ is by definition $B T$, giving the commutative diagram

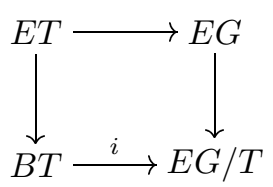

Now $T$ and $G$ have the $k$-point 1 ; it thus follows from Lemma A.2 and Proposition A.5 that the map

$$
i^{*}: H^{*}\left(E G / T, \mathcal{M}_{*}\right) \rightarrow H^{*}\left(B T, \mathcal{M}_{*}\right)
$$

is an isomorphism. This holds more generally when replacing $T$ by a reductive subgroup whose torsors are locally trivial for the Zariski topology (e.g. a product 
of $\mathbb{G}_{m}, S L(n)$ and $S p(2 n)$ ), or by any reductive subgroup if we replace Zariski cohomology by étale cohomology.

\section{Appendix B. The Rost invariant}

Let $H$ be a semi-simple, simply connected linear algebraic group over $k$. If $H$ is split, we have an isomorphism $H_{\text {Zar }}^{2}\left(B H, \mathcal{K}_{2}\right) \stackrel{\sim}{\rightarrow} \mathbf{S}^{2}\left(L_{H}\right)^{W}$ by Theorem 4.8 , where $T$ is a split maximal torus, $L_{H}=\operatorname{Hom}\left(T, \mathbb{G}_{m}\right)$ and where $W$ is the Weyl group of $H$. By Lemma 6.4, we therefore have a canonical isomorphism:

$$
\mathbb{H}_{\text {ét }}^{4}(B H, \Gamma(2)) \simeq H^{3}(k, \mathbb{Q} / \mathbb{Z}(2)) \oplus \mathbf{S}^{2}\left(L_{H}\right)^{W} .
$$

If $H$ is simple, the group $\mathbf{S}^{2}\left(L_{H}\right)^{W}$ is known to be free of rank 1. We show that this situation extends to the non-split case in a straightforward way.

B.1. Proposition. Let $H$ be a (not necessarily split) semi-simple, simply connected linear algebraic group over $k$. Let $X$ • be a smooth simplicial scheme over $k$ and $E_{\bullet} \rightarrow X_{\bullet}$ be an $H$-torsor (this means that, for each $n, E_{n} \rightarrow X_{n}$ is an $H$-torsor, and that all faces and degeneracies preserve the torsor structures). Then there are isomorphisms

$$
\mathbb{H}_{\text {êt }}^{i}\left(X_{\bullet}, \Gamma(2)\right) \stackrel{\sim}{\rightarrow} \mathbb{H}_{\text {êt }}^{i}\left(E_{\bullet}, \Gamma(2)\right)(i \leq 2)
$$

and an exact sequence

$$
\begin{aligned}
0 \rightarrow \mathbb{H}_{\text {êt }}^{3}\left(X_{\bullet}, \Gamma(2)\right) \rightarrow \mathbb{H}_{\text {êt }}^{3}\left(E_{\bullet}, \Gamma(2)\right) \rightarrow H_{\text {êt }}^{0}\left(X_{\bullet}, \mathbf{H}^{1}\left(E_{\bullet}, \mathcal{K}_{2}\right)\right) & \\
& \rightarrow \mathbb{H}_{\mathrm{êt}}^{4}\left(X_{\bullet}, \Gamma(2)\right) \rightarrow \mathbb{H}_{\text {êt }}^{4}\left(E_{\bullet}, \Gamma(2)\right)
\end{aligned}
$$

where $\mathbf{H}^{1}\left(E_{\bullet}, \mathcal{K}_{2}\right)$ is the simplicial sheaf defined as follows: its component over $X_{n}$ is the étale sheaf associated to the presheaf $U \mapsto H_{\text {Zar }}^{1}\left(E_{n} \times_{X_{n}} U, \mathcal{K}_{2}\right)$. This simplicial sheaf is locally constant.

To prove this proposition, we use the following lemma:

B.2. Lemma. Let $X$ be a smooth variety over $k$ and $E$ be an $H$-torsor on $X$. Let

$$
\Gamma_{\text {ét }}(\pi, 2)=\operatorname{cone}\left(\Gamma_{\text {ét }}(X, 2) \rightarrow R \pi_{*} \Gamma_{\text {ét }}(E, 2)\right)
$$

where $\pi: E \rightarrow X$ is the projection. Then the cohomology sheaves $\mathcal{H}^{i}\left(\Gamma_{\text {ét }}(\pi, 2)\right)$ are:

- 0 for $i \leq 2$;

- the (locally constant) sheaf $\mathbf{H}^{1}\left(E, \mathcal{K}_{2}\right)$ defined as in Proposition B.1 for $i=3$.

Proof. We have an exact sequence of étale sheaves

$$
\begin{aligned}
0 \rightarrow \mathcal{H}^{0}\left(\Gamma_{\text {ét }}(\pi, 2)\right) & \rightarrow \mathcal{H}^{1}\left(\Gamma_{\text {ét }}(X, 2)\right) \rightarrow R^{1} \pi_{*} \Gamma_{\text {ét }}(E, 2) \\
& \rightarrow \mathcal{H}^{1}\left(\Gamma_{\text {ét }}(\pi, 2)\right) \rightarrow \mathcal{H}^{2}\left(\Gamma_{\text {ét }}(X, 2)\right) \rightarrow R^{2} \pi_{*} \Gamma_{\text {ét }}(E, 2) \rightarrow \cdots
\end{aligned}
$$

By [40] (and [33, lemma 1.4 (ii)]), the étale sheaf $\mathcal{H}^{q}\left(\Gamma_{\text {ét }}(X, 2)\right)$ is 0 for $q \neq 1,2$, and its stalk at a geometric point $x \in X$ is $K_{3}\left(K_{x}^{s h}\right)_{\text {ind }}$ for $q=1$ (resp. $K_{2}\left(\mathcal{O}_{X, x}^{s h}\right)$ for $q=2$ ), where $\mathcal{O}_{X, x}^{s h}$ is the strict henselization of $\mathcal{O}_{X}$ at $x$ and $K_{x}^{s h}$ is its field of fractions. Since $H$ is locally split and $E$ is locally trivial for the étale topology, the stalks of $R^{q} \pi_{*} \Gamma_{\text {ét }}(E, 2)$ for $q \leq 4$ are given by (6.1), (6.2) and Corollary 3.22: for a geometric point $x$ of $X$, we have

- $R^{1} \pi_{*} \Gamma_{\text {ét }}(E, 2)_{x}=K_{3}\left(K_{x}^{s h}\right)_{\text {ind }}$;

- $R^{2} \pi_{*} \Gamma_{\text {ét }}(E, 2)_{x}=K_{2}\left(\mathcal{O}_{X, x}^{s h}\right)$;

- $R^{3} \pi_{*} \Gamma_{\text {ét }}(E, 2)_{x}=H_{\text {Zar }}^{1}\left(\mathcal{O}_{X, x}^{s h} \times_{X} E, \mathcal{K}_{2}\right)=H_{\text {Zar }}^{1}\left(K_{x}^{s h} \times_{X} E, \mathcal{K}_{2}\right)$. 
To get the first isomorphism, note that by Lemma $6.2, K_{3}(K)_{\text {ind }} \stackrel{\sim}{\rightarrow} K_{3}(K(H))_{\text {ind }}$ for all $K$, since $H$ is a geometrically connected rational variety. To get the second isomorphism, use Corollary 3.22 for $M_{*}=K_{*}^{M}$ and $i=2$ plus Gersten's conjecture for $K_{2}$. Similarly for the third isomorphisms. Note that, if $H_{\text {split }}$ is the split semisimple group associated to $H, \mathbf{H}^{1}\left(E, \mathcal{K}_{2}\right)$ is locally isomorphic to the constant sheaf $H_{\text {Zar }}^{1}\left(H_{\text {split }}, \mathcal{K}_{2}\right)$.

All this gives $\mathcal{H}^{0}\left(\Gamma_{\text {ét }}(\pi, 2)\right)=0$ and the rest of the sequence as

$$
\begin{aligned}
0 \rightarrow \mathcal{H}^{1}\left(\Gamma_{\text {ét }}(\pi, 2)\right) \rightarrow \mathcal{K}_{2} \rightarrow \mathcal{K}_{2} \rightarrow \mathcal{H}^{2}\left(\Gamma_{\text {ét }}(\pi, 2)\right) & \\
& \rightarrow 0 \rightarrow \mathbf{H}^{1}\left(H, \mathcal{K}_{2}\right) \rightarrow \mathcal{H}^{3}\left(\Gamma_{\text {ét }}(\pi, 2)\right) \rightarrow 0 .
\end{aligned}
$$

Proposition B.1 now follows from Lemma B.2 by noting that

$$
\mathbb{H}_{\text {ét }}^{3}\left(X_{\bullet}, \Gamma\left(\pi_{\bullet}, 2\right)\right) \simeq H_{\text {ét }}^{0}\left(X_{\bullet}, \mathbf{H}^{1}\left(E_{\bullet}, \mathcal{K}_{2}\right)\right)
$$

where $\pi_{\bullet}: E_{\bullet} \rightarrow X_{\bullet}$ is the projection and $\Gamma\left(\pi_{\bullet}, 2\right)$ is the simplicial complex of sheaves with components $\Gamma\left(\pi_{n}, 2\right)$.

B.3. Corollary. Let $H$ be as in Proposition B.1 and $\bar{H}=H \times_{k} k_{s}$, where $k_{s}$ is a separable closure of $k$. Then $K_{2}(k) \stackrel{\sim}{\rightarrow} H_{\text {Zar }}^{0}\left(H, \mathcal{K}_{2}\right)$ and there is a commutative square of isomorphisms

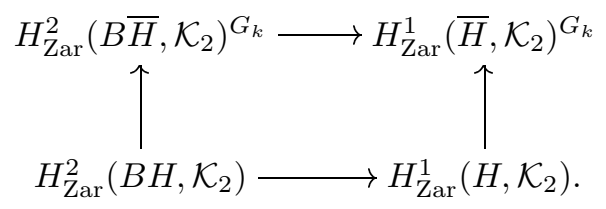

Proof. The vertical homomorphisms in the diagram are induced by extension of scalars. Applying Proposition B.1 to the torsor $H \rightarrow$ Spec $k$ and using Proposition 3.20 (i) and (6.1) proves the first claim. This also follows from Proposition 3.20 (i), together with Suslin's result [63] that $K_{2}(k(X)) / K_{2}(k)$ satisfies Galois descent.

Noting that the unit section splits the map $H \rightarrow$ Spec $k$, Proposition B.1 and (6.1) show that the right hand vertical arrow is an isomorphism.

Now consider the spectral sequence (4.1) for $\mathcal{K}_{2}$ on both $B H$ and $B \bar{H}$. We know from the proof of Theorem 4.7 that $E_{1}^{1,1}=E_{2}^{1,1}$ for $B \bar{H}$. Replacing $H$ with $H^{2}$, we have shown above that the map

$$
H_{\text {Zar }}^{1}\left(H^{2}, \mathcal{K}_{2}\right) \rightarrow H_{\text {Zar }}^{1}\left(\bar{H}^{2}, \mathcal{K}_{2}\right)^{G_{k}}
$$

is an isomorphism, hence $E_{1}^{1,1}=E_{2}^{1,1}$ for $B H$ as well. The first claim applied to $H^{p}$ shows that the $E_{2}^{p, 0}$ terms are zero, hence the maps

$$
H_{\text {Zar }}^{2}\left(B \bar{H}, \mathcal{K}_{2}\right) \rightarrow H_{\text {Zar }}^{1}\left(\bar{H}, \mathcal{K}_{2}\right)
$$

and

$$
H_{\text {Zar }}^{2}\left(B H, \mathcal{K}_{2}\right) \rightarrow H_{\text {Zar }}^{1}\left(H, \mathcal{K}_{2}\right)
$$

exist and are isomorphisms. The commutativity of the diagram then follows from the naturality of the spectral sequence (4.1).

B.4. Lemma. Suppose that in Proposition B.1, $H$ is absolutely simple. Then the sheaf $\mathbf{H}^{1}\left(E_{\bullet}, \mathcal{K}_{2}\right)$ is constant, with value $\mathbb{Z}$. 
Proof. We first deal with the case $H=S L(N)$. Then, for each $n, E_{n} \rightarrow X_{n}$ is locally trivial for the Zariski topology. It follows that the Zariski sheaf associated to the presheaf $U \mapsto H_{\text {Zar }}^{1}\left(E_{n} \times_{X_{n}} U, \mathcal{K}_{2}\right)$ is locally isomorphic to the constant sheaf with value $H^{1}\left(S L(N), \mathcal{K}_{2}\right) \simeq \mathbb{Z}$, hence is itself constant with value $\mathbb{Z}$. The same is a fortiori true for the corresponding étale sheaf.

In general, let $\rho: H \rightarrow S L(N)$ be a nontrivial representation defined over $k$, and let $\rho_{*} E_{\bullet}$ be the induced torsor over $X_{\bullet}$. The map $H_{\text {Zar }}^{1}\left(S L(N) \times_{k} k_{s}, \mathcal{K}_{2}\right) \rightarrow$ $H_{\mathrm{Zar}}^{1}\left(\bar{H}, \mathcal{K}_{2}\right)$ is nontrivial (see below). Since both groups are infinite cyclic, it is injective. It follows that the natural map of étale sheaves $\mathbf{H}^{1}\left(\rho_{*} E_{\bullet}, \mathcal{K}_{2}\right) \rightarrow$ $\mathbf{H}^{1}\left(E_{\bullet}, \mathcal{K}_{2}\right)$ is a monomorphism. Since both sheaves are locally isomorphic to $\mathbb{Z}$ and the first one is constant, the second one must be constant too.

B.5. Theorem. Let $H$ be a simple simply connected algebraic group over $k$. Then:

a) There is an isomorphism

$$
\mathbb{H}_{\text {ét }}^{4}(B H, \Gamma(2)) \simeq H_{\text {ét }}^{3}(k, \mathbb{Q} / \mathbb{Z}(2)) \oplus \mathbb{Z} .
$$

b) For $X_{\bullet}, E_{\bullet}$ as in Proposition B.1, with $X_{0}$ geometrically connected, the exact sequence of Proposition B.1 simplifies to

$$
0 \rightarrow \mathbb{H}_{\text {ét }}^{3}\left(X_{\bullet}, \Gamma(2)\right) \rightarrow \mathbb{H}_{\text {êt }}^{3}\left(E_{\bullet}, \Gamma(2)\right) \rightarrow \mathbb{Z} \stackrel{\alpha}{\rightarrow} \mathbb{H}_{\text {êt }}^{4}\left(X_{\bullet}, \Gamma(2)\right) \rightarrow \mathbb{H}_{\text {êt }}^{4}\left(E_{\bullet}, \Gamma(2)\right) .
$$

Moreover, if $Y_{\bullet} \stackrel{f}{\rightarrow} X_{\bullet}$ is a map of smooth simplicial $k$-schemes, with $Y_{0}$ geometrically connected, and $F_{\bullet}=f^{*} E_{\bullet}$, then the map

$$
H_{\text {ét }}^{0}\left(X_{\bullet}, \mathbf{H}^{1}\left(E_{\bullet}, \mathcal{K}_{2}\right)\right) \rightarrow H_{\text {ét }}^{0}\left(Y_{\bullet}, \mathbf{H}^{1}\left(F_{\bullet}, \mathcal{K}_{2}\right)\right)
$$

is an isomorphism.

c) If $X_{\bullet}$ is either constant or satisfies the assumptions of Lemma 6.4 a), then this exact sequence can be rewritten

$$
0 \rightarrow H_{\text {Zar }}^{1}\left(X_{\bullet}, \mathcal{K}_{2}\right) \rightarrow H_{\text {Zar }}^{1}\left(E_{\bullet}, \mathcal{K}_{2}\right) \rightarrow \mathbb{Z} \stackrel{\alpha}{\rightarrow} \mathbb{H}_{\text {ét }}^{4}(X \bullet, \Gamma(2)) \rightarrow \mathbb{H}_{\text {êt }}^{4}\left(E_{\bullet}, \Gamma(2)\right) .
$$

Proof. The hypothesis on $H$ implies that $H \simeq R_{\ell / k} H^{\prime}$ where $H^{\prime}$ is absolutely simple, $\ell / k$ is a finite separable extension and $R_{\ell / k}$ denotes Weil's restriction of scalars [65, p. 46]. By Corollary 3.21, we have

$$
H_{\text {Zar }}^{1}\left(\bar{H}, \mathcal{K}_{2}\right) \simeq \operatorname{Ind}_{G_{\ell}}^{G_{k}} H_{\text {Zar }}^{1}\left(\bar{H}^{\prime}, \mathcal{K}_{2}\right)
$$

as Galois modules, and Lemma B.4 shows that $G_{\ell}$ acts trivially on $H_{\mathrm{Zar}}^{1}\left(\bar{H}^{\prime}, \mathcal{K}_{2}\right)$. Therefore, $H_{\text {Zar }}^{1}\left(\bar{H}, \mathcal{K}_{2}\right)$ is a permutation module under $G_{k}$ and $H_{\text {Zar }}^{1}\left(\bar{H}, \mathcal{K}_{2}\right)^{G_{k}} \simeq$ $H_{\text {Zar }}^{1}\left(H^{\prime}, \mathcal{K}_{2}\right) \simeq \mathbb{Z}$. a) follows from this, together with Corollary B.3 and Lemma 6.4 .

To prove b), we observe that there exists up to isomorphism a unique $H^{\prime}$-torsor $E_{\bullet}^{\prime}$ over $X_{\bullet}^{\prime}:=X_{\bullet} \otimes_{k} \ell$ such that $E_{\bullet} \simeq f_{*} E_{\bullet}^{\prime}$, where $f: X_{\bullet}^{\prime} \rightarrow X_{\bullet}$ is the projection. Then $\mathbf{H}^{1}\left(E_{\bullet}, \mathcal{K}_{2}\right) \simeq f_{*} \mathbf{H}^{1}\left(E_{\bullet}^{\prime}, \mathcal{K}_{2}\right) \simeq f_{*} \mathbb{Z}$, hence

$$
H_{\text {ét }}^{0}\left(X_{\bullet}, \mathbf{H}^{1}\left(E_{\bullet}, \mathcal{K}_{2}\right)\right) \simeq H_{\text {ét }}^{0}\left(X_{\bullet}^{\prime}, \mathbb{Z}\right)=H_{\text {ét }}^{0}\left(X_{0}^{\prime}, \mathbb{Z}\right)=\mathbb{Z}
$$

since $X_{0}^{\prime}=X_{0} \times_{k} l$ is connected. The last claim of b) is obvious, by a similar argument. Finally, c) follows from b) and (6.1) or Lemma 6.4. (The computation $H_{\text {Zar }}^{1}\left(H, \mathcal{K}_{2}\right) \simeq \mathbb{Z}$ is due to Deligne [12].)

B.6. Let $H$ be simple simply connected and let $\rho: H \rightarrow S L(N)$ be some nontrivial representation as above. If $k=\mathbb{C}$, the map $H_{\text {Zar }}^{2}\left(B S L(N), \mathcal{K}_{2}\right) \stackrel{\rho *}{\rightarrow} H_{\text {Zar }}^{2}\left(B H, \mathcal{K}_{2}\right)$ is nontrivial. An easy way to see this is to use Theorem 4.11 to reduce to topology, 
in which case the result is well-known. If $H$ is split, the same holds by reduction to the complex case (Theorem 4.11). This is still the case for general $H$, as one sees by passing to the separable closure of $k$.

If $\rho^{\prime}$ is another such representation of $H$, then $\rho^{*} c_{2}$ and $\rho^{\prime *} c_{2}$ differ in $H^{2}\left(B H, \mathcal{K}_{2}\right)$ $\simeq \mathbb{Z}$ by a positive rational number. To see this, embed $\rho$ and $\rho^{\prime}$ into $\rho+\rho^{\prime}$. This reduces us to the case in which $\rho^{\prime}=\lambda \circ \rho$ for some $\lambda: S L(N) \rightarrow S L\left(N+N^{\prime}\right)$; then it can be checked that $\lambda^{*} c_{2}$ is a positive multiple of $c_{2}$ by reducing to the fundamental representations of $S L(N)$ (alternatively, reduce to topology). It follows that there is a unique generator $\gamma_{H}$ of $H_{\mathrm{Zar}}^{2}\left(B H, \mathcal{K}_{2}\right)$ such that, for $\rho: H \rightarrow S L(N)$ a homomorphism of algebraic groups,

$$
\rho^{*} c_{2}=d_{\rho} \gamma_{H}
$$

where $d_{\rho}$ is a positive integer. Note that the string of isomorphisms

$$
H_{\text {Zar }}^{2}\left(B H, \mathcal{K}_{2}\right) \stackrel{\sim}{\rightarrow} H_{\text {Zar }}^{1}\left(H, \mathcal{K}_{2}\right) \stackrel{\sim}{\rightarrow} H_{\text {Zar }}^{1}\left(\bar{H}, \mathcal{K}_{2}\right)^{G_{k}}=H_{\text {ét }}^{0}\left(B H, \mathbf{H}^{1}\left(E H, \mathcal{K}_{2}\right)\right)
$$

(compare Corollary B.3) yields a canonical generator of the three other groups, that we still denote by $\gamma_{H}$.

Let $d_{H}$ be the greatest common divisor of the integers $d_{\rho}$. If $H$ is split, these multipliers are clearly independent of $k$; they were computed explicitly by Dynkin [16] in the case $k=\mathbb{C}$ for analytic cohomology, with a few mistakes for $H=E_{8}$, corrected by Freudenthal [18] and others (see [25] and [36, prop. 2.6]). It turns out that, at least in the split case, $d_{H}$ is always realized by a certain fundamental $S L$-representation $\psi$ of $H$.

For the reader's convenience, we recall the list of Dynkin indices of split simple groups, together with the weight of a fundamental representation $\psi$ such that $d_{\psi}=d_{H}$ (compare [25] or [36, prop. 2.6]):

\begin{tabular}{||l|c|c|c|c|c|c|c|c|c||}
\hline$H$ & $A_{r}$ & $B_{r}, r \geq 3$ & $C_{r}$ & $D_{r}, r \geq 4$ & $E_{6}$ & $E_{7}$ & $E_{8}$ & $F_{4}$ & $G_{2}$ \\
\hline$d_{H}$ & 1 & 2 & 1 & 2 & 6 & 12 & 60 & 6 & 2 \\
\hline weight of $\psi$ & $\varpi_{1}$ & $\varpi_{1}$ & $\varpi_{1}$ & $\varpi_{1}$ & $\varpi_{6}$ & $\varpi_{7}$ & $\varpi_{8}$ & $\varpi_{4}$ & $\varpi_{1}$ \\
\hline
\end{tabular}

The reader should take special care with $D_{3}$, corresponding to $\operatorname{Spin}(3,3)$, which is not in this table. In fact, $D_{3}$ is isomorphic to $A_{3}$ and $\operatorname{Spin}(3,3)$ is accordingly isomorphic to $S L(4)$, so its Dynkin index is 1 . However the index associated to the representation $\psi$ of Theorem 5.8 is 2 even for $n=3$ and Theorem 5.8 is correct as stated.

Let $X$ be a $k$-scheme and $E$ an $H$-torsor on $X$, as in Proposition B.1. Then $E$ is locally trivial for the étale topology of $X$ and $\gamma_{H}$ yields a characteristic class

$$
\gamma_{H}(E)=[E]^{*} \gamma_{H} \in \mathbb{H}_{\text {ét }}^{4}(X, \Gamma(2))
$$

where $[E] \in[X, B H]_{\text {ét }}$ is the homotopy class associated to $E$. We have:

B.7. Lemma. Let $\rho: H \rightarrow S L(N)$ be a linear representation of $H$, and let $V=$ $\rho_{*} E$ be the associated vector bundle. Then

$$
\rho^{*} c_{2}(V)=d_{\rho} \gamma_{H}(E)
$$

where $d_{\rho}$ is the multiplier described in B.6.

Suppose now that $X=\operatorname{Spec} k$. Then $H_{\text {ét }}^{3}(k, \mathbb{Q} / \mathbb{Z}(2)) \rightarrow \mathbb{H}_{\text {êt }}^{4}(k, \Gamma(2))$ is an isomorphism. Denote by $e(E)$ the inverse image of $\gamma_{H}(E)$ in $H_{\text {ét }}^{3}(k, \mathbb{Q} / \mathbb{Z}(2))$ : this is the Rost invariant of $E$. 
B.8. Proposition. For any E over Spec $k$, we have

$$
d_{H} e(E)=0
$$

where $d_{H}$ is the Dynkin index of $H$.

Proof. This is obvious from Lemma B.7.

As in [33, end of introduction], let

$$
\mathbb{Z} / d_{H}(2)= \begin{cases}\mu_{d_{H}}^{\otimes 2} & \text { if } \operatorname{char} k=0, \\ \mu_{d_{H}^{\prime}}^{\otimes 2} \oplus W_{r} \Omega_{\log }^{2}[-2] & \text { if } \operatorname{char} k=p>0\end{cases}
$$

where (if char $k=p>0$ ) $d_{H}^{\prime}$ is the prime-to- $p$ part of $d_{H}$ and $W_{r} \Omega_{\log }^{2}$ is the weighttwo logarithmic part of the de Rham-Witt complex at length $r$, where $p^{r} \| d_{H}$. From the Merkurjev-Suslin theorem [46] (and the Bloch-Gabber-Kato theorem at the characteristic [4, Corollary 2.8]), the sequence

$$
0 \rightarrow H_{\text {ét }}^{3}\left(k, \mathbb{Z} / d_{H}(2)\right) \rightarrow H_{\text {ét }}^{3}(k, \mathbb{Q} / \mathbb{Z}(2)) \stackrel{d_{H}}{\rightarrow} H_{\text {ét }}^{3}(k, \mathbb{Q} / \mathbb{Z}(2))
$$

is exact. So the Rost invariant refines into an invariant in $H_{\mathrm{èt}}^{3}\left(k, \mathbb{Z} / d_{H}(2)\right)$.

Let $X$ be a smooth variety over $k$ and $E$ an $H$-torsor over $X$. One sees as in section 8 that the component $e\left(E_{\eta}\right)$ in $H_{\text {êt }}^{3}\left(k(X), \mathbb{Z} / d_{H}^{\prime}(2)\right)$ is unramified over $X$. We can then show the following analogue to Theorem 6.11 , exactly in the same way as above:

B.9. Theorem. Let $\rho: H \rightarrow S L(N)$ be a representation of $H$, and let $e^{\prime}\left(E_{\eta}\right) \in$ $H^{0}\left(X, \mathcal{H}^{3}\left(\mu_{d_{\rho}^{\prime}}^{\otimes 2}\right)\right)$ be the prime-to-the-characteristic part of $e\left(E_{\eta}\right)$, viewed in the group $H^{0}\left(X, \mathcal{H}^{3}\left(\mu_{d_{\rho}^{\prime}}^{\otimes 2}\right)\right)$ where $d_{\rho}^{\prime}$ is the prime-to-p part of $d_{\rho}$. Then

$$
d_{2}\left(e^{\prime}\left(E_{\eta}\right)\right)=c_{2}(E) \in C H^{2}(X) / d_{\rho}^{\prime}
$$

where $c_{2}(E)$ is the second Chern class of the vector bundle deduced from $E$ via the representation $\rho$.

If it happens that $d_{\rho}=d_{H}$, this theorem gives a computation of $d_{2}\left(e^{\prime}\left(E_{\eta}\right)\right) \in$ $C H^{2}(X) / d_{H}$, viewing $e^{\prime}\left(E_{\eta}\right)$ as an element of $H^{0}\left(X, \mathcal{H}^{3}\left(\mu_{d_{H}^{\prime}}^{\otimes 2}\right)\right)$.

We conclude this section with a proof of Rost's announced theorem. When $H=$ Spin, this allows this paper to be self-contained.

B.10. Proposition. Let $H$ be simple, simply connected and let $E \rightarrow X$ be an $H$ torsor on a smooth $k$-scheme $X$. Then, with notation as in Theorem B.5 b), we have $\alpha(1)=\gamma_{H}(E)$. In particular,

$$
\operatorname{Ker}\left(\mathbb{H}_{\text {êt }}^{4}(X, \Gamma(2)) \rightarrow \mathbb{H}_{\text {êt }}^{4}(E, \Gamma(2))\right)=\left\langle\gamma_{H}(E)\right\rangle .
$$

Proof. This follows from the commutative diagram, coming from Theorem B.5 c):

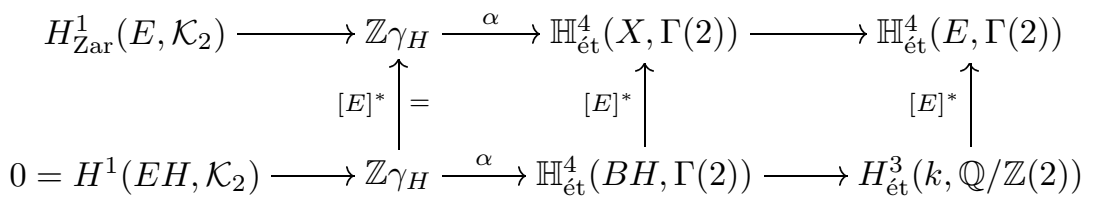

(note that, by Lemma A.2, $\mathbb{H}_{\text {Zar }}^{1}\left(E H, \mathcal{K}_{2}\right)=0$ and the composite $\mathbb{H}_{\text {êt }}^{4}(k, \Gamma(2)) \rightarrow$ $\mathbb{H}_{\text {ét }}^{4}(B H, \Gamma(2)) \rightarrow \mathbb{H}_{\text {êt }}^{4}(E H, \Gamma(2))$ is an isomorphism). 
B.11. Theorem (Rost). Let $H$ be a simple, simply connected algebraic group over $k$. Let $E$ be an $H$-torsor on $k$ and let $K=k(E)$ be its function field. Let $\eta$ be the map $H_{\text {èt }}^{3}(k, \mathbb{Q} / \mathbb{Z}(2)) \rightarrow H_{\text {êt }}^{3}(K, \mathbb{Q} / \mathbb{Z}(2))$ given by extension of scalars. Then

$$
\operatorname{Ker} \eta=\langle e(E)\rangle \text {. }
$$

Proof. Note that $\bar{E} \simeq \bar{H}$ and that $K_{2}(\bar{k}) \stackrel{\sim}{\rightarrow} H^{0}\left(\bar{H}, \mathcal{K}_{2}\right)$, as follows from Proposition 3.20. We therefore have an exact sequence extending that of Theorem B.5 c) (for $\left.X_{\bullet}=\operatorname{Spec} k\right)$ :

$$
H^{1}\left(E, \mathcal{K}_{2}\right) \rightarrow\left(H^{1}\left(\bar{E}, \mathcal{K}_{2}\right)^{G_{F}} \simeq \mathbb{Z}\right) \alpha>>\operatorname{Ker} \eta \rightarrow \operatorname{Ker}\left(C H^{2}(E) \rightarrow C H^{2}(\bar{E})\right) .
$$

(This exact sequence follows from [9, prop. 3.6] and [32, th. 3.1], see [53] or [33, th. 1].) We have $C H^{2}(E)=C H^{2}(\bar{E})=0$ : as has already been pointed out, the case of $\bar{E} \simeq \bar{H}$ follows from [38, th. 2.1], and the general case of $E$ is [51, cor. 5.2 (4)]. Finally, the equality $\alpha\left(\gamma_{H}\right)=e(E)$ is a special case of Theorem B.5 c) (for $\left.X_{\bullet}=\operatorname{Spec} k\right)$.

\section{Appendix C. An amusing example}

Let $H$ be as above. We apply Proposition B.10 to the following "generic" case: let $\rho: H \rightarrow S L(N)$ be a faithful linear representation of $H$. To $\rho$ and $r \geq 1$ we associate the $k$-variety

$$
B_{r} H=\frac{S L(N+r)}{H \times S L(r)}
$$

where $H$ is identified with its image in $S L(N)$. We also associate the vector bundle $H^{\Delta_{l}} \times k^{N} / \rho$ on $B H$, and its class $\rho^{*} c_{2} \in \mathbb{H}_{\text {êt }}^{4}(B H, \Gamma(2))$.

The variety $B_{r} H$ is smooth and carries a tautological $H$-torsor $E=S L(N+r) /$ $S L(r)$. As before, $E$ determines a homotopy class of map

$$
[E] \in\left[B_{r} H, B H / k\right]_{\text {ét }} \text {. }
$$

C.1. Theorem. a) For $r \geq 2$, the map

$$
\mathbb{H}_{\text {ét }}^{4}(B H, \Gamma(2)) \stackrel{[E]^{*}}{\longrightarrow} \mathbb{H}_{\text {ét }}^{4}\left(B_{r} H, \Gamma(2)\right)
$$

is injective, with p-primary torsion cokernel, where $p$ is the characteristic exponent of $k$ (so, in characteristic 0 , it is an isomorphism).

b) For $r=1$, there is a split exact sequence, up to p-primary torsion groups

$$
0 \rightarrow H^{3}(k, \mathbb{Q} / \mathbb{Z}(2)) \rightarrow \mathbb{H}^{4}\left(B_{1} H, \Gamma(2)\right) \rightarrow \mathbb{Z} / d_{\rho} \rightarrow 0
$$

and (C.1) has p-primary torsion cokernel. Its kernel is generated by $\rho^{*} c_{2}$.

Proof. We first compute $H_{\text {Zar }}^{*}\left(E, \mathcal{K}_{2}\right)$ for $r \geq 2$. We could go via the Leray spectral sequence of the fibration $\pi: S L(N+r) \rightarrow E$ (for the Zariski cohomology), using the fact that any $S L(r)$-bundle is locally trivial for the Zariski topology and using Corollary 3.22 as above. It is perhaps more elegant to go back to cycle cohomology and use Rost's spectral sequence [57, cor. 8.2]:

$$
E_{2}^{p, q}=A^{p}\left(E, A^{q}\left[\pi, K_{2}\right]\right) \Rightarrow A^{p+q}\left(S L(N+r), K_{2}\right)
$$

where $\pi: S L(N+r) \rightarrow E$ is the projection (the two arguments are essentially the same anyway). $A^{q}\left[\pi, K_{j}\right]$ is defined by

$$
A^{q}\left[\pi, K_{j}\right](K)=A^{q}\left(S L(N+r) \times_{E} \operatorname{Spec} K, K_{j}\right)
$$


for any point Spec $K \rightarrow E$. It is a cycle module, since the fibration $\pi$ is a $S L(r)$ torsor, its fiber is trivial at all such points and in particular $A^{q}\left[\pi, K_{j}\right](K) \simeq$ $A^{q}\left(S L(r) / K, K_{j}\right)$ for any $K$. The spectral sequence gives an exact sequence

$$
\begin{aligned}
0 \rightarrow A^{1}\left(E, A^{0}\left[\pi, K_{2}\right]\right) \rightarrow A^{1}(S L & \left.(N+r), K_{2}\right) \\
& \rightarrow A^{0}\left(E, A^{1}\left[\pi, K_{2}\right]\right) \rightarrow A^{2}\left(E, A^{0}\left[\pi, K_{2}\right]\right) \rightarrow 0
\end{aligned}
$$

noting that $A^{2}\left(S L(N+r), K_{2}\right)=0$ in view of Proposition 3.20. Still from Proposition 3.20 , we get $A^{0}\left[\pi, K_{0}\right]=K_{0}, A^{0}\left[\pi, K_{1}\right]=K_{1}, A^{1}\left[\pi, K_{1}\right]=0$ and

$$
A^{q}\left[\pi, K_{2}\right] \simeq \begin{cases}K_{2}, & q=0, \\ \mathbb{Z} c_{2}(\text { constant }), & q=1, \\ 0, & q \geq 2 .\end{cases}
$$

Therefore

$$
A^{0}\left(E, A^{1}\left[\pi, K_{2}\right]\right)=H_{\mathrm{Zar}}^{0}\left(E, \mathbb{Z} c_{2}\right)=\mathbb{Z} c_{2}
$$

and the map

$$
A^{1}\left(S L(N+r), K_{2}\right)=\mathbb{Z} c_{2} \rightarrow A^{0}\left(E, A^{1}\left[\pi, K_{2}\right]\right)=\mathbb{Z} c_{2}
$$

is an isomorphism as it is an isomorphism while restricting to the generic point of $E$. Thus both $A^{1}\left(E, A^{0}\left[\pi, K_{2}\right]\right)$ and $A^{2}\left(E, A^{0}\left[\pi, K_{2}\right]\right)$ are zero. Since

$A^{1}\left(E, A^{0}\left[\pi, K_{2}\right]\right)=H^{1}\left(K_{2}(k(E)) \rightarrow \bigoplus_{x \in E^{(1)}} K_{1}(k(x)) \rightarrow \bigoplus_{x \in E^{(2)}} \mathbb{Z}\{x\}\right)=A^{1}\left(E, K_{2}\right)$

and

$A^{2}\left(E, A^{0}\left[\pi, K_{2}\right]\right)=H^{2}\left(K_{2}(k(E)) \rightarrow \bigoplus_{x \in E^{(1)}} K_{1}(k(x)) \rightarrow \bigoplus_{x \in E^{(2)}} \mathbb{Z}\{x\}\right)=A^{2}\left(E, K_{2}\right)$

we get $A^{1}\left(E, K_{2}\right)=A^{2}\left(E, K_{2}\right)=0$. By $(6.2)$, it follows that

$$
\mathbb{H}_{\text {ét }}^{4}(E, \Gamma(2)) \stackrel{\sim}{\rightarrow} H_{\text {Zar }}^{0}\left(E, \mathcal{H}^{3}(\mathbb{Q} / \mathbb{Z}(2))\right) \text {. }
$$

On the other hand,

$$
H^{3}\left(k, \mathbb{Q}_{l} / \mathbb{Z}_{l}(2)\right) \stackrel{\sim}{\rightarrow} H_{\text {Zar }}^{0}\left(E, \mathcal{H}^{3}\left(\mathbb{Q}_{l} / \mathbb{Z}_{l}(2)\right)\right)
$$

for all $l \neq p$. To see this, apply the Rost spectral sequence to the cycle module $K \mapsto H^{*}\left(K, \mathbb{Q}_{l} / \mathbb{Z}_{l}(*-1)\right)$ and use Proposition 3.20 (i). Hence the map

$$
\mathbb{H}_{\text {ét }}^{4}(k, \Gamma(2)) \rightarrow \mathbb{H}_{\text {ét }}^{4}(E, \Gamma(2))
$$

has $p$-primary torsion cokernel; and this map is injective since $E$ has a rational point.

For $r \geq 2$, Theorem C.1 now follows from diagram (B.1). Finally, in the case $r=1$, we have $E=S L(N+1)$ and Proposition 3.20 shows that $A^{i}\left(E, K_{2}\right)=$ $K_{2}(k), \mathbb{Z} c_{2}$ or 0 according as $i=0,1$ or 2 , and the conclusion again follows from diagram (B.1).

In contrast to Theorem C.1, the Zariski cohomology groups of $B H$ and $B_{r} H$ are in general "different", as the following corollary shows. 
C.2. Corollary. For $r \geq 2$,

a) There is an exact sequence

$$
\begin{aligned}
0 \rightarrow C H^{2}\left(B_{r} H\right) \otimes \mathbb{Z}[1 / p] \stackrel{\mathrm{cl}^{2}}{\longrightarrow} H_{\text {Zar }}^{2}( & \left.B H, \mathcal{K}_{2}\right) \otimes \mathbb{Z}[1 / p] \\
& \rightarrow \frac{H_{\text {ét }}^{0}\left(B_{r} H, \mathcal{H}^{3}(\mathbb{Q} / \mathbb{Z}(2))\right)}{H_{\text {êt }}^{3}(k, \mathbb{Q} / \mathbb{Z}(2))} \otimes \mathbb{Z}[1 / p] \rightarrow 0
\end{aligned}
$$

where, as before, $p$ is the characteristic exponent of $k$. This exact sequence realizes $C H^{2}\left(B_{r} H\right) \otimes \mathbb{Z}[1 / p]$ as a subgroup of index $d_{H}$ of $H_{\text {Zar }}^{2}\left(B H, \mathcal{K}_{2}\right) \otimes \mathbb{Z}[1 / p]=$ $\mathbb{Z}[1 / p] \gamma_{H}$.

b) We have:

$$
H_{\text {ét }}^{0}\left(B_{r} H, \mathcal{H}^{3}(\mathbb{Q} / \mathbb{Z}(2))\right) \otimes \mathbb{Z}[1 / p] \simeq H_{\text {ét }}^{3}(k, \mathbb{Q} / \mathbb{Z}(2)) \otimes \mathbb{Z}[1 / p] \oplus \mathbb{Z}[1 / p] e\left(E_{\eta}\right)
$$

where $e\left(E_{\eta}\right)$ is the Rost invariant of the generic fiber of the $H$-torsor $E$; this invariant has order $d_{H}$.

Proof. We assume in the sequel that everything has been tensored by $\mathbb{Z}[1 / p]$. The first claim of a) follows easily from Theorem C.1, the diagram with exact rows

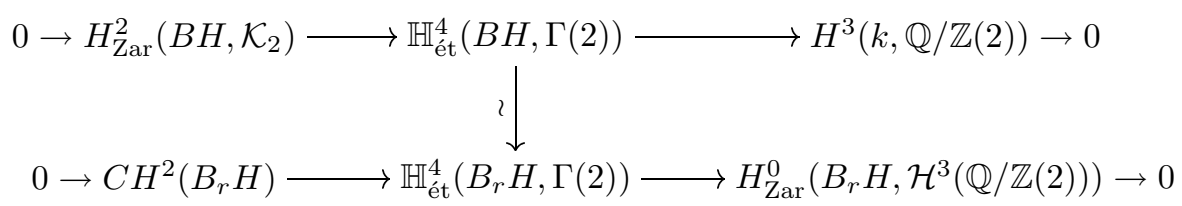

and the fact that $H^{3}(k, \mathbb{Q} / \mathbb{Z}(2)) \rightarrow H_{\mathrm{Zar}}^{0}\left(B_{r} H, \mathcal{H}^{3}(\mathbb{Q} / \mathbb{Z}(2))\right)$ is split injective, since $B_{r} H$ has a rational point. (The top row is a split exact sequence by Lemma 6.4.) On the other hand, b) follows from a).

It remains therefore to prove the second claim of a). This is equivalent to a statement proved by Rost (see [60, p. 783-15, exemple]). We shall give a simple proof of it, based on a recent result of Merkurjev [45]. We first need a well-known lemma:

C.3. Lemma. Let $X$ be a smooth variety over $k$. Then $C H^{2}(X)$ is generated by the $c_{2}(E)$, where $E$ runs through the algebraic vector bundles over $X$ of determinant 1.

Proof. Consider the composition (for all $i \geq 0$ )

$$
C H^{i}(X) \stackrel{\mathrm{Cl}^{i}}{\longrightarrow} K_{0}(X) \stackrel{c_{i}}{\longrightarrow} C H^{i}(X)
$$

in which $\mathrm{Cl}^{i}$ is the $K$-theoretic cycle class map and $c_{i}$ is the $i$-th Chern class with values in the Chow group. It follows from Riemann-Roch without denominators [31] that $c_{i} \circ \mathrm{Cl}^{i}=(-1)^{i-1}(i-1) ! I d_{C H^{i}(X)}[24$, formula (4.5) and further comments]. In particular, for $i=2$, this composition is minus the identity. To obtain bundles of determinant 1 , one replaces $E$ by $E \oplus \operatorname{det}(E)^{-1}$.

By $[45$, cor. 6.6], the natural map

$$
R(H) \rightarrow K_{0}\left(B_{1} H\right)
$$

given by the "Borel construction" is surjective. Here $R(H)$ is the representation ring of $H$. Together with Lemma C.3, this shows that $C H^{2}\left(B_{1} H\right)$ is generated by 
the $\psi^{*} c_{2}$, where $\psi$ runs through the special linear representations of $H$. Consider now the commutative diagram

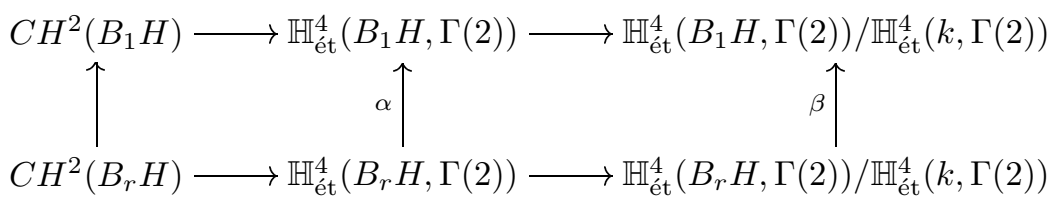

where $r \geq 2$. By Theorem C.1 a), the bottom composition coincides with the map $\mathrm{cl}^{2}$ of Corollary C.2. By the above remark and the surjectivity of the left vertical map (Theorem C.1), the image of $\beta \circ \mathrm{cl}^{2}$ is the subgroup generated by the $\psi^{*} c_{2}$. But, by Theorem C.1 b), $\alpha$ is surjective with kernel generated by $\rho^{*} c_{2}$. So the same conclusion holds for the image of $\mathrm{cl}^{2}$, which therefore has index $d_{H}$, by definition of the Dynkin index of $H$.

C.4. Question. The class $e\left(E_{\eta}\right)$ is unramified over $B_{r} H$. Consider a smooth compactification $X$ of $B_{r} H$. Is $e\left(E_{\eta}\right)$ unramified over the whole of $X$ ?

\section{ACKNOWLEDGEMENTS}

It will be clear to the reader that Rost's ideas permeate this article. In particular, his suggestion that one could use motivic cohomology to construct his invariants was a key insight for our proof. We also thank Burt Totaro and Jean-Pierre Serre for useful comments. Finally we thank the referee for pointing out several ambiguities and for his thorough remarks.

\section{REFERENCES}

[1] J. Kr. Arason, Cohomologische Invarianten quadratischer Formen, J. Alg. 36 (1975), 448491. MR 52:10592

[2] J. Barge, Une definition cohomologique de l'invariant d'Arason, preprint, 1995.

[3] S. Bloch and A. Ogus, Gersten's conjecture and the homology of schemes, Ann. Sci. Ecole Norm. Sup. 7 (1974), 181-202. MR 54:318

[4] S. Bloch and K. Kato, p-adic étale cohomology, Publ. Math. IHES 63 (1986), 107-152. MR 87k:14018

[5] R. Bott On torsion in Lie groups, Proc. Acad. Sci. USA 40 (1954), 586-588. MR 16:12a

[6] N. Bourbaki, Eléments de Mathématiques, Groupes et Algèbres de Lie, ch. 4,5,6, Masson, Paris, 1981. MR 83g:17001

[7] C. Chevalley, Sur les décompositions cellulaires des espaces G/B, Proc. Sympos. Pure Math. 56 (I), AMS, Providence, 1994, 1-23. MR 95e:14041

[8] J.-L. Colliot-Thélène, Birational invariants, purity and Gersten's conjecture, Proc. Sympos. Pure Math. 58.1, AMS, Providence, 1995, 1-64. MR 96c: 14016

[9] J.-L. Colliot-Thélène and W. Raskind, sK $K_{2}$-cohomology and the second Chow group, Math. Ann. 270 (1985), 165-199. MR 86m:14005

[10] J.-L. Colliot-Thélène, R. Hoobler, and B. Kahn, The Bloch-Ogus-Gabber theorem, to appear in: Proc. Fields Institute (Volume in memory of R. Thomason).

[11] P. Deligne, Théorie de Hodge, III, Publ. Math. IHES 44 (1974), 5-78. MR 58:16653b

[12] P. Deligne, unpublished notes of IHES lectures, 1979.

[13] M. Demazure and A. Grothendieck, Séminaire de géométrie algébrique du Bois-Marie: Schémas en groupes (SGA 3), tome III, Lecture notes in Math. 153, Springer, Berlin, 1970. MR 43:223c

[14] M. Demazure, Invariants symétriques du groupe de Weyl et torsion, Invent. Math. 21 (1973), 287-301. MR 49:7268

[15] M. Demazure, Désingularisation des variétés de Schubert généralisées, Ann. Sci. ENS 7 (1974), 53-68. MR 50:7174 
[16] E. B. Dynkin, Semisimple subalgebras of semisimple Lie algebras, Mat. Sbornik N.S. 30(72) (1952), 349-462. Engl. translation: AMS Transl. Ser. II 6 (1957), 111-244. MR 13:904c

[17] H. Esnault, B. Kahn, and E. Viehweg, Coverings with odd ramification and Stiefel-Whitney classes, J. reine angew. Math. 441 (1993), 145-188. MR 94m:14017

[18] H. Freudenthal, Zur Berechnung der Charaktere der halbeinfachen Lieschen Gruppen. II, Indag. Math. 16 (1954), 487-491. MR 1:673a

[19] W. Fulton, Intersection theory, Erg. Math. 2, Springer, 1984. MR 85k:14004

[20] O. Gabber, Gersten's conjecture for some complexes of vanishing cycles, Manuscripta Math. 85 (1994), 323-343. MR 96m:14010

[21] H. Gillet, Riemann-Roch theorems for higher algebraic K-theory, Adv. in Math. 40 (1981), 203-289. MR 83m:14013

[22] M. Gros, Classes de Chern et classes de cycles en cohomologie de Hodge-Witt logarithmique, Mém. Soc. Math. France 21 (1985). MR 87m:14021

[23] A. Grothendieck, Torsion homologique et sections rationnelles, exposé 5 in Séminaire Chevalley, "Anneaux de Chow et applications", Paris, 1958.

[24] A. Grothendieck, Problèmes ouverts en théorie des intersections, exposé XIV in Théorie des intersections et théorème de Riemann-Roch (SGA6), Lect. Notes in Math. 225, Springer, 1971, 667-689. MR 50:7133

[25] B. Harris, Torsion in Lie goups and related spaces, Topology 5 (1966), 347-354. MR 34:6798

[26] J.E. Humphreys, Linear algebraic groups (corrected third printing), Springer, New York, 1987. MR 53:633 (original 1975 printing)

[27] L. Illusie, Complexe cotangent et déformations I, Lect. Notes in Math. 239, Springer, Berlin, 1971. MR 58:10886a

[28] W. Jacob and M. Rost, Degree four cohomological invariants for quadratic forms, Invent. Math. 96 (1989), 551-570. MR 90g:11044

[29] U. Jannsen, Mixed Motives and Algebraic K-Theory, Lecture Notes in Mathematics 1400, Springer, Berlin-Heidelberg, 1990. MR 91g:14008

[30] J.F. Jardine, Higher spinor classes, Mem. Amer. Math. Soc. 110 (1994), no. 528. MR 95a: 11035

[31] J.-P. Jouanolou, Riemann-Roch sans dénominateurs, Invent. Math. 11 (1970), 15-26. MR 48:11115

[32] B. Kahn, Descente galoisienne et $K_{2}$ des corps de nombres, $K$-theory 7 (1993), 55-100. MR 94i:11094

[33] B. Kahn, Applications of weight-two motivic cohomology, Documenta Math. 1 (1996), 395416. CMP 97:05

[34] A. Laghribi, Isotropie de certaines formes quadratiques de dimension 7 et 8 sur le corps des fonctions d'une quadrique, Duke Math. J. 85 (1996), 397-410. MR 97h:11039

[35] T.Y. Lam, The algebraic theory of quadratic forms (2nd ed.), Benjamin, New York, 1980. MR 83d: 10022

[36] Y. Laszlo and C. Sorger, The line bundles on the moduli of parabolic G-bundles over curves and their sections, Ann. Sci. Ec. Norm. Sup. (4) 30 (1997), no. 4, 499-525. CMP 97:14

[37] M. Levine, The indecomposable $K_{3}$ of fields, Ann. Sci. Ec. Norm. Sup. 22 (1989), 255-344. MR 91a:11061

[38] M. Levine, The algebraic K-theory of the classical groups and some twisted forms, Duke Math. J. 70 (1993), 405-443. MR 94d:19004

[39] S. Lichtenbaum, Values of zeta-functions at non-negative integers, Lect. Notes in Math. 1068, Springer, Berlin, 1984, 127-138. CMP 16:17

[40] S. Lichtenbaum, The construction of weight-two arithmetic cohomology, Invent. Math. 88 (1987), 183-215. MR 88d:14011

[41] S. Lichtenbaum, New results on weight-two motivic cohomology, The Grothendieck Festschrift, vol. 3, Progress in Math. 88, Birkhaüser, Boston, 1990, 35-55. MR 92m:14030

[42] R. Marlin, Anneaux de Chow des groupes algébriques $S U(n), S p(n), S O(n), \operatorname{Spin}(n), G_{2}$, $F_{4}$; torsion, C. R. Acad. Sci. Paris 279 (1974), 119-122. MR 50:321

[43] A. S. Merkurjev, On the norm residue symbol of degree 2, Dokl. Akad. Nauk SSSR 261 (1981), 542-547. English translation: Soviet Math. Dokl. 24 (1981), 546-551. MR 83h:12015

[44] A. S. Merkurjev, The group $H^{1}\left(X, \mathcal{K}_{2}\right)$ for projective homogeneous varieties (in Russian), Algebra i Analiz 7 (1995), 136-164. English translation: Leningrad (Saint-Petersburg) Math. J. 7 (1996), 421-444. MR 97a:19003 
[45] A. S. Merkurjev, Comparison of equivariant and ordinary $K$-theory of algebraic varieties, to appear in St.-Petersburg Math. J.

[46] A. S. Merkurjev and A. A. Suslin, $\mathcal{K}$-cohomology of Severi-Brauer varieties and norm residue homomorphism (in Russian), Izv. Akad. Nauk SSSR 46 (1982), 1011-1046. English translation: Math USSR Izv. 21 (1983), 307-340. MR 84i:12007

[47] A. S. Merkurjev and A. A. Suslin, The norm residue homomorphism of degree 3 (in Russian), Izv. Akad. Nauk SSSR 54 (1990), 339-356. English translation: Math. USSR Izv. 36 (1991), 349-368. MR 91f: 11083

[48] A. S. Merkurjev and A. A. Suslin, The group $K_{3}$ for a field (in Russian), Izv. Akad. Nauk. SSSR 54 (1990), 522-545. English translation: Math. USSR Izv. 36 (1991), 541-565. MR 91g:19002

[49] J. W. Milnor and J. D. Stasheff, Characteristic classes, Annals of Mathematics Studies 76, Princeton University Press, Princeton, 1974. MR 55:13428

[50] I. A. Panin, Application of $K$-theory in algebraic geometry, doctoral dissertation, LOMI, Leningrad, 1984.

[51] I. A. Panin, A splitting principle, Preprint, Bielefeld University, 1994.

[52] R. Parimala and V. Srinivas, Analogues of the Brauer group for algebras with involution, Duke Math. J. 66 (1992), 207-237. MR 93i:16027

[53] E. Peyre, Corps de fonctions de variétés homogènes et cohomologie galoisienne, C. R. Acad. Sci. Paris 321 (1995), 891-896. MR 96i:14006

[54] D. Quillen, Higher algebraic K-theory, I, Lect. Notes in Math. 341, Springer, New York, 1973, 83-147. MR 49:2895

[55] P. Roquette, The Galois cohomology of the projective linear group, Math. Ann. 150 (1963), 411-439. MR 27:4832

[56] M. Rost, Hilbert's theorem 90 for $K_{3}^{M}$ for degree-two extensions, preprint, Regensburg, 1986.

[57] M. Rost, Chow groups with coefficients, Documenta Math. 1 (1996), 319-393. CMP 97:04

[58] M. Rost, Cohomological invariants, in preparation.

[59] W. Scharlau, Quadratic and hermitian forms, Springer, Berlin, 1985. MR 86k:11022

[60] J-P. Serre, Cohomologie galoisienne: progrès et problèmes, Sém. N. Bourbaki, march 1994, exposé 783. MR 97d:11063

[61] C. S. Seshadri, Line bundles on Schubert varieties, Vector bundles on algebraic varieties (Bombay, 1984), T.I.F.R. Studies in Math. 11, Oxford University Press, 1987, 499-528. MR 88i: 14047

[62] A. A. Suslin, $K$-theory and $\mathcal{K}$-cohomology of certain group varieties, Adv. in Soviet Math. 4, AMS, Providence, 1991, 53-74. MR 92g:19004

[63] A. A. Suslin, Torsion in $K_{2}$ of fields, $K$-theory 1 (1987), 5-29. MR 89a:11123

[64] M. Szyjewski, The fifth invariant of quadratic forms (in Russian), Algebra Anal. 2 (1990), 213-234. English translation: Leningrad Math. J. 2 (1991), 179-198. MR 91d:11040

[65] J. Tits, Classification of algebraic semi-simple groups, Proc. Sympos. Pure Math. 9, AMS, 1966, 33-62. MR 37:309

FB6, Mathematik, Universität Essen, D-45117 Essen, Germany

E-mail address: esnault@uni-essen.de

Institut de Mathématiques de Jussieu, Université Paris 7, Case 7012, 75251 Paris Cedex 05, France

E-mail address: kahn@math.jussieu.fr

Department of Mathematics, Northeastern University, Boston, Massachusetts 02115

E-mail address: marc@neu.edu

FB6, Mathematik, Universität Essen, D-45117 Essen, Germany

E-mail address: viehweg@uni-essen.de 